\title{
Nearly ordinary rank four Galois representations and $p$-adic Siegel modular forms
}

\author{
J. Tilouine
}

With an appendix by Don Blasius

\begin{abstract}
This paper is devoted to the proof of two results. The first was conjectured in 1994 by the author. It concerns the identity, under certain assumptions, of the universal deformation ring of $p$-nearly ordinary Galois representations and a local component of the universal nearly ordinary Hecke algebra in the sense of Hida. The other, which relies on the first, concerns the modularity of certain abelian surfaces. More precisely, one can associate to certain irreducible abelian surfaces defined over the rationals overconvergent $p$-adic cusp eigenforms. The question of whether these forms are classical is not studied in this paper.
\end{abstract}

\section{Contents}

Introduction . . . . . . . . . . . . . . . . . . . . 1122

1 Some background and motivation . . . . . . . . . . . . . . . 1124

2 Universal nearly ordinary objects . . . . . . . . . . . . . . . . 1130

3 An $R=$ T theorem . . . . . . . . . . . . . . . . . . . . . 1134

4 p-adic Siegel modular forms . . . . . . . . . . . . . . 1138

5 Abelian surfaces . . . . . . . . . . . . . . . . . . . 1148

Appendix. A rank 3 generalization of the conjecture of Shimura

and Taniyama . . . . . . . . . . . . . . . . . . 1151

References . . . . . . . . . . . . . . . . . . 1154

\section{Introduction}

This paper has two goals. First, we deduce from a recent result [GT05] the proof, for the case $F=\mathbb{Q}$ and under certain assumptions, of two conjectures proposed ten years ago [Til96] on nearly ordinary deformations of the Galois representation associated to an ordinary cohomological Siegel cusp form of genus two (Theorem 4 below). Second, we apply this result to show that certain nearly ordinary degree four symplectic Galois representations with singular Hodge-Tate weights that are congruent to a modular Galois representation are $p$-adically modular. This shows in particular that certain abelian surfaces $A / \mathbb{Q}$ do correspond, if they are residually modular, to a $p$-adic Siegel cusp form of (diagonal) weight 2 (see Theorem 8).

More precisely, let $\pi$ be a cuspidal representation occurring in the middle degree cohomology of the (adelic) Siegel threefold of level group $K$, with coefficients in a local system of highest weight $(a, b), a \geqslant b \geqslant 0$. Let $p$ be a prime, prime to the level of $K$ and such that $p-1>a+b+3$; let $\mathbf{T}$ be the localization at the maximal ideal corresponding to $(\pi, p)$ of the universal nearly ordinary

Received 10 February 2005, accepted in final form 11 January 2006.

2000 Mathematics Subject Classification 11F33, 11F46, 11F80 (primary), 11G18, 11F70 (secondary).

Keywords: Galois representations, Hecke algebras, Siegel varieties, congruences, $p$-adic modular forms.

This journal is (c) Foundation Compositio Mathematica 2006. 


\section{NEARLY ORDINARY RANK FOUR GALOIS REPRESENTATIONS}

Hecke algebra (see [Hid90, TU99, MT02]). It is naturally an algebra over the Hida-Iwasawa algebra $\Lambda$, which is an Iwasawa algebra in three variables. On the other hand, let $R$ be the universal deformation ring of nearly ordinary symplectic Galois representations congruent to the residual Galois representation associated to $(\pi, p)$ (which is assumed to be symplectic). It also comes with a natural structure of $\Lambda$-algebra. It turns out that a twist of this natural structure is more adapted to our needs (Definition 2.2); it is called the canonical $\Lambda$-algebra structure of $R$. The conjectures concern the Krull dimension of $R$ and the existence of a canonical isomorphism of local $\Lambda$-algebras between $R$, with its canonical structure, and $\mathbf{T}$, together with their finiteness and flatness over $\Lambda$. As usual, we first construct a surjective algebra homomorphism $\phi: R \rightarrow \mathbf{T}$ by the universal property of $R$; for the natural $\Lambda$-algebra structure on $\mathbf{T}$ and the canonical structure on $R$, it turns out to be $\Lambda$-linear. In order to prove its injectivity together with the finiteness and flatness of these $\Lambda$-algebras, we introduce a faithful $\mathbf{T}$-module $\mathbf{V}$ that is finite free over $\Lambda$. We recall an exact control theorem for $\mathbf{V}$ at cohomological weights [TU99, MT02] and we apply it to the weight of the cohomological form $\pi$. We then show, under an assumption that we call strict ordinarity for $(\pi, p)$, that one can apply an isomorphism theorem proven by Genestier and Tilouine [GT05] to this specialization. The role of this assumption is unfortunately crucial for the moment. In fact, in [GT05], the assumption that the representations are crystalline at $p$ is necessary, due to our lack of knowledge on the $p$-decomposition subgroup action on the étale cohomology of a Siegel variety of level divisible by $p$. The strict ordinarity assumption assures that any ordinary deformation of the residual representation associated to $(\pi, p)$ is actually crystalline. We then conclude that $\phi$ is bijective by a nice, but rather well-known, use of Nakayama's lemma (§3.2).

One then considers geometric Galois representations that are $p$-nearly ordinary with HodgeTate weights of the form $\left(0, \ell_{0}-2, k_{0}-1, \ell_{0}+k_{0}-3\right)$ for an arbitrary pair $\left(k_{0}, \ell_{0}\right) \in \mathbb{Z}^{2}$ such that $2 \leqslant \ell_{0}, 1 \leqslant k_{0}$ and $\ell_{0}+k_{0}-3<p-1$. By specializing the isomorphism $\phi: R \cong \mathbf{T}$ to the weight $\left(a_{0}, b_{0}\right)=\left(k_{0}-3, \ell_{0}-3\right)$, we conclude that such a representation comes from a $p$-adic Hecke eigensystem of weight $\kappa_{0}=\left(\ell_{0}, k_{0}\right)$. We show, by using a theorem of Hida [Hid02], that although the weight $\kappa_{0}$ is not cohomological, this eigensystem does correspond to a $p$-adic false modular form $f_{0}$ of weight $\left(k_{0}, \ell_{0}\right)$, belonging to a $p$-adic family of nearly ordinary Siegel cusp forms. We then establish a conjecture of Hida ([Hid02, Conjecture 7.2]) in a particular case but in a stronger form (Theorem 6). If we furthermore assume that $k_{0}=\ell_{0}=2$, this allows us to prove the overconvergence of $f_{0}$. More precisely, let $E$ be an arbitrary lifting of a certain power $H^{t}$ of the Hasse invariant (for some integer $t$ prime to $p$ ) to the Siegel threefold of level $K$. By using a result of Abbès-Mokrane [AM04], we prove that the overconvergence of $f_{0}$ holds in the domain $|E(x)|_{p}>r$ of the rigid Siegel threefold of level $K$ (prime-to- $p$ ) for some radius $r \in] p^{-1 / p^{2}(p-1)}, 1[$. One expects that $r$ can be taken arbitrarily close from the lower bound. Moreover, Abbès-Mokrane lower bound $p^{-1 / p(p-1)}$ has been recently improved in [AG03] to $p^{-(p-1) /(2 p-1)}$.

Our theory of overconvergence relies on this result together with the technique of Kisin-Lai [KL05]. We recently became aware of a note by Lai and Zhao [LZ] exclusively based on [KL05], which presents the notion of overconvergent Siegel modular forms in a way slightly different from ours.

The question whether $f_{0}$ is classical is not addressed in this paper. We hope to come back to this type of question later.

As an application, we can show that certain abelian surfaces defined over $\mathbb{Q}$, semistable outside $p$, with bad reduction at $p$ but acquiring ordinary good reduction over $\mathbb{Q}_{p}\left(\zeta_{p}\right)$ at $p$, if they are residually modular, do come from overconvergent $p$-adic Siegel cusp forms, in the sense that their Galois representations are conjugated in $\operatorname{GSp}_{4}\left(\overline{\mathbb{Q}}_{p}\right)$.

There is a conjectural list of triples $\left(G, X, \pi_{\infty}\right)$ consisting of a reductive group $G / \mathbb{Q}$ admitting a Shimura variety $S$ locally isomorphic to the hermitian symmetric domain $X$, and an admissible representation $\pi_{\infty}$ of $G(\mathbb{R})$ occurring in the coherent cohomology of $S$, such that 


\section{J. Tilouine}

the set of Galois representations associated to the cuspidal representations with infinity type $\pi_{\infty}$ is conjecturally identical to that associated to (submotives with coefficients of) abelian varieties with specific polarizations and endomorphism rings. Although the complete list is not known to us, we conclude the paper by quoting some of its items in order to put our result in perspective. An appendix by D. Blasius at the end of this article provides another such item. It gives a partial answer to a question of the referee.

\section{Some background and motivation}

Let

$$
G=\mathrm{GSp}(4)=\left\{X \in \mathrm{GL}_{4} ;{ }^{\mathrm{t}} X J X=\nu \cdot J\right\}
$$

be the split reductive group scheme over $\mathbb{Z}$ of symplectic simitudes for the antisymmetric matrix $J$, given by its $2 \times 2$ block decomposition: $J=\left(\begin{array}{cc}0 & s \\ -s & 0\end{array}\right)$ where $s$ is the $2 \times 2$ antidiagonal matrix whose nonzero coefficients are equal to 1 . This group comes with a canonical character $\nu: X \mapsto \nu(X) \in \mathbb{G}_{m}$, called the similitude factor, and a canonical cocharacter $i: z \mapsto z \cdot 1_{4}$, called the center cocharacter (here $1_{4}$ is the unit matrix). The center of $G$ is denoted by $Z$, the standard (diagonal) maximal torus by $T$ and the standard (upper triangular) Borel by $B ; N$ denotes its unipotent radical, so that $B=T N$. Let $\gamma_{P}=t_{1} / t_{2}$ (respectively $\gamma_{Q}=\nu^{-1} t_{2}^{2}$ ) be the short (respectively long) simple root associated to the triple $(G, B, T)$. The standard maximal parabolic $P=M U$, associated to $\gamma_{P}$, is called the Klingen parabolic, while the standard maximal parabolic $Q=M^{\prime} U^{\prime}$, associated to $\gamma_{Q}$, is the Siegel parabolic. The Weyl group of $G$ is denoted $W_{G}$. It is generated by the two reflections

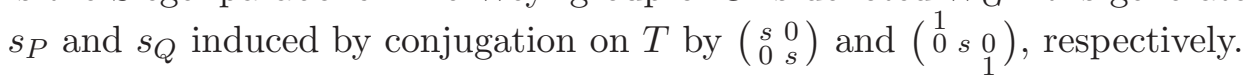

Let us fix a pair of integers $(a, b) \in \mathbb{Z}^{2}, a \geqslant b \geqslant 0$; we identify it with a dominant weight for $(G, B, T)$, namely the character

$$
T \ni t=\operatorname{diag}\left(t_{1}, t_{2}, \nu^{-1} t_{2}, \nu^{-1} t_{1}\right) \mapsto t_{1}^{a} t_{2}^{b} .
$$

Let $V_{a, b}$ be a generically irreducible algebraic representation of $G$ associated to $(a, b)$ over $\mathbb{Z}$.

\subsection{Two conjectures on Galois representations associated to Siegel-Hilbert cusp eigensystems}

Consider a totally real field $F$ of degree $d$ over $\mathbb{Q}$.

Let $\pi$ be a cohomological cuspidal representation of $G\left(\mathbb{A}_{F}\right)$ occurring in the middle degree cohomology group $H^{3 d}\left(S, V_{a, b}(\mathbb{C})\right)$ where $S$ is the Shimura (pro-)variety of $G\left(\mathbb{A}_{F}\right)$ and $V_{a, b}(\mathbb{C})$ is the local system on $S$ associated to $V_{a, b}$ (see [TU99, $\left.\S 1\right]$ ). The data $(G, B, T)$ give rise to root data $\left(\Delta, \Phi, \Delta^{\vee}, \Phi^{\vee}\right)$, as in [Bor79]. Let $\widehat{G}$ be the dual reductive group scheme over $\mathbb{Z}$ of $G$, associated to the dual root data. It comes equipped with a Borel $\widehat{B}$, a maximal torus $\widehat{T}$ and a canonical cocharacter $\widehat{\nu}$ and a canonical character $\hat{i}$.

Remark. It turns out that $\widehat{G}=\mathrm{GSpin}_{5}$ is isomorphic by the spin representation spin: $\mathrm{GSpin}_{5} \rightarrow \mathrm{GL}_{4}$ to $G$ itself. In this identification, one has $\widehat{i}=\nu$ and $\widehat{\nu}=i$ (see [Bor79, $\S 1$ ], or the considerations preceding Definition 2.2 below). However, it will be apparent in the formulation of the conjectures below that it is useful to distinguish between $G$ and $\widehat{G}$ as corresponding to the two sides (automorphic and Galois) of the Langlands correspondence.

Assume that the compatible system of degree four Galois representations $\left(R_{\pi, \ell}\right)$ of $\operatorname{Gal}(\bar{F} / F)$ associated to such a cohomological cuspidal representation has been constructed and that for each prime $\ell, R_{\pi, \ell}$ factors through $\widehat{G}\left(\overline{\mathbb{Q}}_{\ell}\right)$ via the spin representation. Let $p$ be a fixed odd prime; fix an isomorphism of $\overline{\mathbb{Q}}_{p}$ with $\mathbb{C}$. Let $S$ be the set of ramification of $\pi$ and $S_{p}$ be the set of primes of $F$ above $p$; let $\Gamma_{F}$ be the Galois group of the maximal extension of $F$ contained in $\bar{F}$ and unramified outside $S \cup S_{p}$. 


\section{NeARLY ORDinARY RANK FOUR Galois REPRESENTATIONS}

Thus, there exists a continuous homomorphism

$$
\rho_{\pi, p}: \Gamma_{F} \rightarrow \widehat{G}\left(\overline{\mathbb{Q}}_{p}\right)
$$

such that for any $v \notin S \cup S_{p}, \rho_{\pi, p}\left(F r_{v}\right)$ is conjugated to the Satake parameter of $\pi$ at $v$ in $\widehat{G}(\mathbb{C})$.

Remark. (1) If $\rho_{\pi, p}$ is constructed, its similitude factor $\widehat{i} \circ \rho_{\pi, p}$ is known on Frobenius substitutions hence everywhere by the Chebotarev density theorem. Indeed, one must have $\widehat{i} \circ \rho_{\pi, p}=\epsilon^{-\mathbf{w}} \omega_{\pi}$ where $\epsilon$ is the $p$-adic cyclotomic character, $\mathbf{w}$ is the motivic weight of $\pi$ and $\omega_{\pi}$ the finite-order character given by the central character of $\pi$. An argument of parity implies in particular that $\rho_{\pi, p}$ is totally odd: for any complex conjugation $c$ in $\Gamma_{F}, \widehat{i} \circ \rho_{\pi, p}(c)=-1$.

(2) The system of Galois representation $\left(R_{\pi, \ell}\right)_{\ell}$ associated to $\pi$ is constructed only when $F=\mathbb{Q}$ at this time. Moreover, one does not know whether it preserves a symplectic form.

Let $\mathcal{O}$ be a discrete valuation subring of $\overline{\mathbb{Z}}_{p}$, finite over $\mathbb{Z}_{p}$, sufficiently big so that $\operatorname{Im} \rho_{\pi, p} \subset \widehat{G}(\mathcal{O})$. Let $\varpi$ be a uniformizing parameter and $k=\mathcal{O} /(\varpi)$ the residue field. Let $\bar{\rho}: \Gamma_{F} \rightarrow \widehat{G}(k)$ be the reduction of $\rho_{\pi, p}$ modulo $\varpi$. We say that $\bar{\rho}$ is a modular residual representation. We know that it is totally odd, that is, for any complex conjugation $c$ in $\Gamma_{F}, \widehat{i} \circ \bar{\rho}(c)=-1$.

Let us assume that the following assumptions are satisfied.

(1) $\bar{\rho}$ is absolutely irreducible.

(2) $\bar{\rho}$ is nearly ordinary, that is, for any $v \in S_{p}$, and for any decomposition group $D_{v}$ at $v$ in $\Gamma_{F}$, $\bar{\rho}\left(D_{v}\right)$ is contained in a conjugate in $\widehat{G}(k)$ of the Borel subgroup $\widehat{B}(k)$; say, $\bar{g}_{v} \bar{\rho}\left(D_{v}\right) \bar{g}_{v}^{-1} \subset \widehat{B}(k)$. We denote by $\phi_{\bar{\rho}, v}: D_{v} \rightarrow \widehat{T}(k)$ the homomorphism defined by $\bar{g}_{v}\left(\bar{\rho} \mid D_{v}\right) \bar{g}_{v}^{-1} \bmod \widehat{N}(k)$.

For simplicity in this introduction, we do not specify the type of local conditions (called $S$-good in Definition 1.3 below) that we put on the ramification at places $v$ in $S$. It is enough to say that we call a deformation $\rho$ of $\bar{\rho}$ 'minimal at $v$ ' if it satisfies 'the same type of ramification condition as $\bar{\rho}$. We will make them explicit in the main theorem, when $F=\mathbb{Q}$.

Let us consider the assumptions of regularity:

$$
\text { (Reg) For any simple root } \gamma \text { of } \widehat{G}, \gamma \circ \phi_{\bar{\rho}, v} \text { is not identically } 1
$$

and

$\left(\operatorname{Reg}^{*}\right)$ For any simple root $\gamma$ of $\widehat{G}, \gamma \circ \phi_{\bar{\rho}, v}$ is neither 1 nor $\omega$

where $\omega$ denotes the cyclotomic character modulo $p$.

We consider the deformation problem $\mathcal{F}_{\bar{\rho}}$ defined over the category of complete noetherian local $\mathcal{O}$-algebras $A$ with maximal ideal $\mathfrak{m}$ and residue field $k$, which sends $A$ to the set, modulo strict equivalence, of deformations $\rho: \Gamma_{F} \rightarrow \widehat{G}(A)$ such that (1) they are minimal at each $v \in S$, (2) for any $v \in S_{p}$, there exists a conjugate $g_{v} \rho\left(D_{v}\right) g_{v}^{-1}$ (for a $g_{v} \in \widehat{G}(A)$ ) which is contained in the Borel subgroup $\widehat{B}(A)$ in such a way that the reduction modulo the maximal ideal $\mathfrak{m}_{A}$ of $A$ of the representation $\phi_{\rho, v}: D_{v} \rightarrow \widehat{T}(A)$ given by $g_{v} \bar{\rho} g_{v}^{-1} \widehat{N}(A)$ is exactly $\phi_{\bar{\rho}, v}$.

Under the assumption (Reg), one can apply Schlessinger's criterion to show that this problem is representable by a universal pair $\left(R, \rho_{R}\right)$ (see $[$ Til96, $\left.\S 6]\right)$. Under the assumption $\left(\operatorname{Reg}^{*}\right)$, we showed ([Til96, § 7]) that the universal deformation $\operatorname{ring} R$ satisfies $\operatorname{dim}(R \otimes k) \geqslant 2 d+1$.

We conjectured the following.

Conjecture 1. We conjecture $\operatorname{dim}(R \otimes k)=2 d+1$.

Let $O_{F}$ be the ring of integers of $F$; let $N$ be an ideal of $O_{F}$ and denote by $O_{F}^{N}=\prod_{(\lambda, N)=1} O_{F, \lambda}$ the prime-to- $N$ completion of $O_{F}$. We say that $N$ is a level of $K$ if one has a decomposition $K=K^{N} \times K_{N}$ with $K^{N}=G\left(O_{F}^{N}\right)$. We shall assume also that the set of primes dividing $N$ 


\section{J. Tilouine}

coincides with $S$. Let $C_{F, N p^{r}}^{+}$be the strict ray-class groups of $F$ of conductor $N p^{r}(r \geqslant 0)$ : the natural reduction maps form a projective system; let $C_{F, N p \infty}^{+}$be their inverse limit. Let $O_{F, N}^{\times}$be the group of units of $F$ congruent to $1 \bmod N$; let $O_{F, p}=O_{F} \otimes \mathbb{Z}_{p}$ be the semilocal completion of the ring $O_{F}$; then one has the adelic description of $C_{F, N p^{\infty}}^{+}$:

$$
1 \rightarrow O_{F, p}^{\times} / \bar{O}_{F, N}^{\times} \rightarrow C_{F, N p^{\infty}}^{+} \rightarrow C_{F, N}^{+} \rightarrow 1
$$

We introduce the Hida group of $\left(G_{/ F}, p\right)$ as the amalgamated sum

$$
H=H_{G, F, p}=T\left(O_{F, p}\right) \times_{O_{F, p}^{\times}} C_{F, N p^{\infty}}^{+}
$$

with respect to the center cocharacter $i: O_{F, p}^{\times} \rightarrow T\left(O_{F, p}\right)$ and the quotient map $\mathcal{O}_{F, p}^{\times} \rightarrow \mathcal{O}_{F, p}^{\times} / \overline{\mathcal{O}}_{F, N}^{\times}$.

The group $H$ is a compact commutative $p$-adic Lie group of dimension $2 d+1+\delta$ where $\delta$ denotes the defect to the Leopoldt conjecture for $F$ at $p$. We consider the completed group $\mathcal{O}$-algebra $\mathcal{O}[[H]]$; its relative Krull dimension over $\mathcal{O}$ is $2 d+1+\delta$.

Let us define a structure of $\mathcal{O}[[H]]$-algebra over $R$. We consider the character $\widehat{i} \circ \rho_{R}: \Gamma_{F} \rightarrow R^{\times}$, and for each $v \in S_{p}$ the homomorphism $\phi_{\rho_{R}, v}: D_{v} \rightarrow \widehat{T}(R)$ coming as above from the nearordinarity of $\rho_{R}$ at $v$. One can interpret $\widehat{i} \circ \rho_{R}$ by global class-field theory as a continuous homomorphism $C_{F, N p^{\infty}}^{+} \rightarrow R^{\times}$, while $\phi_{\rho_{R}, v}$ gives rise by local class-field theory (and restriction to the inertia subgroup at $v$ ) to a homomorphism $O_{F, v}^{\times} \rightarrow \widehat{T}(R)$. By the definition of the dual torus, one can reinterpret those morphisms as $T\left(O_{F, v}\right) \rightarrow R^{\times}$. Putting them together, and by compatibility between local and global class-field theory, these homomorphisms give rise to a canonical homomorphism $H \rightarrow R^{\times}$, hence to a structure of $\mathcal{O}[[H]]$-algebra on $R$ (we will be more precise about this canonical homomorphism in $\S 2$ ).

On the other hand, let

$$
M=\lim _{\longleftarrow} H^{3 d}\left(S_{K^{p} \times I_{r}^{+}}, V_{a, b}(\mathcal{O})\right)_{\mathfrak{m}}
$$

where the inverse limit is taken over $r \geqslant 1, I_{r}$ is the Iwahori subgroup of $G\left(O_{F, p}\right)$ of level $p^{r}$, $I_{r}^{+}=\operatorname{Ker}\left(I_{r} \rightarrow T\left(O_{F, p} / p^{r} O_{F, p}\right)\right)$ and $V_{a, b}$ is the local system in which $\pi$ occurs; the localization being taken relative to the maximal ideal $\mathfrak{m}$ of the abstract Hecke algebra outside $S \cup S_{p}$, associated to $(\pi, p)$.

One can define the nearly ordinary part $e M$ of $M$ as the direct factor on which the Hecke operators at $p$ act by automorphisms (see [TU99, $\S 2.4]$ ). The $\mathcal{O}$-algebra $\mathbf{T}$ is then defined as the direct factor associated to $\pi_{p}$ (see $\S 2.2$ below when $F=\mathbb{Q}$ ) of the complete $\mathcal{O}$-algebra generated by the Hecke operators outside $N p$ acting faithfully on $e M$. It is a complete noetherian local $\mathcal{O}$-algebra.

It contains in its group of invertible elements the group of 'local diamond operators' image of $T\left(O_{F, p}\right)=\lim _{r} I_{r} / I_{r}^{+}$acting on the cohomology by normal action. The group $\mathbb{T}^{\times}$contains also the image of the strict ray-class group of $F$ of conductor $N p^{\infty}$ acting by central action. As noted above, the adelic description of $C_{N p^{\infty}}^{+}$provides a canonical homomorphism $O_{F, p}^{\times} / \bar{O}_{F, N}^{\times} \hookrightarrow C_{N p^{\infty}}^{+}$; the two actions coincide on the center $O_{F, p}^{\times} \subset T\left(O_{F, p}\right)$, hence, again, this provides an $\mathcal{O}[[H]]$-algebra structure on $\mathbf{T}$.

Let $H^{+}$be the pro- $p$-Sylow of $H$ and $\Lambda=\mathcal{O}\left[\left[H^{+}\right]\right]$. It is a result of [TU99] (similar to previous results by Hida for Hilbert modular forms) that $\mathbf{T}$ is finite over $\Lambda$.

Conjecture 2. There exists a canonical $\Lambda$-algebra isomorphism $\phi: R \rightarrow \mathbf{T}$ and both rings are finite and flat over the Hida-Iwasawa algebra $\Lambda$.

Remark. Conjectures 1 and 2 together imply that Leopoldt conjecture: $\delta=0$. 


\section{NEARLY ORDINARY RANK FOUR GALOIS REPRESENTATIONS}

However, in the rest of this paper we restrict ourselves to the case $F=\mathbb{Q}$. Our first result (Theorem 4 below) is that, under certain assumptions, these two conjectures hold when $F=\mathbb{Q}$.

\subsection{Known facts and assumptions}

Let $\mathbb{A}=\mathbb{A}_{f} \times \mathbb{Q}_{\infty}$ be the ring of rational adeles. Fix a compact open subgroup $K$ of $G_{f}=G\left(\mathbb{A}_{f}\right)$; let $N \geqslant 1$ be an integer such that $K=K^{N} \times K_{N}$ with $K^{N}=G\left(\mathbb{Z}^{N}\right)$ maximal compact and $K_{N}=\prod_{\ell \mid N} K_{\ell}$ for local components $K_{\ell}$ to be specified later.

Let $\mathcal{H}^{N}$ be the unramified Hecke algebra outside $N$ (that is, the tensor product algebra of the unramified local Hecke algebras at all prime-to- $N$ rational primes); for each rational prime $\ell$ prime to $N$, one defines the abstract Hecke polynomial $P_{\ell} \in \mathcal{H}^{N}[X]$ as the monic degree four polynomial which is the minimal polynomial of the Hecke Frobenius at $\ell$ (see [And87] or [GT05]).

Let $C_{\infty}$ be the subgroup of $G_{\infty}=G\left(\mathbb{Q}_{\infty}\right)$ generated by the standard maximal compact connected subgroup $K_{\infty}$ and by the center $Z_{\infty}$.

For any neat compact open subgroup $L$ of $G\left(\mathbb{A}_{f}\right)$, the adelic Siegel variety of level $L$ is defined as $S_{L}=G(\mathbb{Q}) \backslash G(\mathbb{A}) / L C_{\infty}$; it is a smooth quasi-projective complex threefold. If $L \subset L^{\prime}$ are neat compact open subgroups of $G_{f}$, we have a finite étale transition morphism $\phi_{L, L^{\prime}}: S_{L} \rightarrow S_{L^{\prime}}$.

Let $S=\lim S_{L}$ be the provariety, inverse limit of the filtering system $\left(S_{L}, \phi_{L, L^{\prime}}\right)$. Let $(a, b)$, $a \geqslant b \geqslant 0$ be a dominant weight for $(G, B, T)$ and $V_{a, b / \mathbb{Z}}$ an integral structure of the rational Weyl representation associated to $(a, b)$; recall that the central character of this representation is $z \mapsto z^{a+b}$.

Let $p$ be a rational prime such that $p$ is prime to $N$ and $p-1>a+b+3$ (so, in particular, $p \geqslant 5)$.

For any module $A$ over $\mathbb{Q}$ or $\mathbb{Z}_{p}$, for any compact open subgroup $L \subset G(\widehat{\mathbb{Z}})$ unramified at $p$, we denote by $V_{a, b}(A)$ the local system over $S_{L}$ associated to $V_{a, b} \otimes A$ (see [MT02]).

Let $\pi=\pi_{f} \otimes \pi_{\infty}$ be a cuspidal representation such that $\pi^{K} \neq 0$ and such that $\pi_{\infty}$ is in the discrete series of Harish-Chandra parameter $(a+2, b+1)$. Thus, $\pi$ occurs in $H^{3}\left(S, V_{a, b}(\mathbb{C})\right)^{K}$. We take the liberty to write $H^{3}\left(S_{K}, V_{a, b}(\mathbb{C})\right)$ this space of invariants even if $K$ is not neat. The representation $\pi$ defines a character $\theta_{\pi}: \mathcal{H}^{N} \rightarrow \mathbb{C}$. For any prime $\ell$ prime to $N$, we can specialize the Hecke polynomial $P_{\ell}$ by $\theta_{\pi}$ into a Hecke polynomial $P_{\pi, \ell} \in \mathbb{C}[X]$.

Let $E$ be the number field generated by the eigenvalues of the Hecke operators outside $N$. We fix a $p$-adic embedding $\iota_{p}$ of $\overline{\mathbb{Q}}$; let $F \subset \overline{\mathbb{Q}}_{p}$ be a $p$-adic field containing $\iota_{p}(E)$ (to be fixed later, big enough but of finite degree).

The Galois representation $W_{\pi}=\operatorname{Hom}_{\text {Hecke }}\left(\pi_{f}^{K}, H^{3}\left(S_{K}, V_{a, b}(F)\right)\right)$ is E-rational and pure of Deligne weight $\mathbf{w}=3+a+b$.

Let $S$ be the set of prime divisors of $N$, and $\Gamma$ be the Galois group of the maximal algebraic extension of $\mathbb{Q}$ unramified outside $S$ and $p$. By a series of papers [Tay93, Lau96, Lau05, Wei05], there exists a degree four Galois representation $R_{\pi, p}: \Gamma \rightarrow \mathrm{GL}_{4}\left(\overline{\mathbb{Q}}_{p}\right)$ such that for any $\ell \notin S \cup\{p\}$, $\operatorname{det}\left(X \cdot 1_{4}-R_{\pi, p}\left(F r_{\ell}\right)\right)=P_{\pi, \ell}(X)$.

We take $F$ big enough for $R_{\pi, p}$ to be defined over it. Let $\mathcal{O}$ be the ring of integers of $F, \varpi$ a uniformizing parameter of $\mathcal{O}$ and $k=\mathcal{O} / \varpi \mathcal{O}$ its residue field.

We assume as in [GT05] that $R_{\pi, p}$ satisfies $\left(R L I_{2}\right)$, that is, there exists a subfield $k^{\prime}$ of $k$ such that up to conjugation in $\mathrm{GL}_{4}(k)$ the image of its reduction modulo $(\varpi)$ is contained in $G(k)$ (that is, factors through spin : $\widehat{G}(k) \rightarrow G(k))$, and contains either the derived group of $G\left(k^{\prime}\right)$, or its subgroup $\Theta\left(k^{\prime}\right)$ defined as the stabilizer of a direct sum of two hyperbolic planes.

Let $\bar{\rho}$ be the reduction of $R_{\pi, p}$ modulo $(\varpi)$. Recall that this assumption implies that all modular deformations $R_{\pi^{\prime}, p}$ of $\bar{\rho}$ are symplectic, absolutely irreducible, and that the corresponding cuspidal cohomological representations $\pi^{\prime}$ are stable at $\infty$ (see [GT05]). 


\section{J. Tilouine}

From now on, unless otherwise specified, we identify $R_{\pi^{\prime}, p}$ to the continuous homomorphism $\rho_{\pi^{\prime}, p}: \Gamma \rightarrow \widehat{G}(\overline{\mathbb{Q}})$ through the isomorphism spin: $\widehat{G} \rightarrow G$.

In particular, we consider the representation $\rho_{p i, p}$, respectively $\bar{\rho}$, respectively its deformations $\rho$ over $\mathcal{O}$-algebras $A$, as taking values in $G(\mathcal{O})$, respectively $G(k)$, respectively $G(A)$.

Remark. (1) Let $\epsilon: \Gamma \rightarrow \mathbb{Z}_{p}^{\times}$be the $p$-adic cyclotomic character. With the convention above, we have $\nu \circ \rho_{\pi, p}=\epsilon^{-\mathbf{w}} \cdot \omega_{\pi}$, where $\omega_{\pi}$ is a finite-order character modulo $N$, given as the Galois avatar of the central character of $\pi$ (this can viewed using Poincaré duality for $W_{\pi}$; see, for instance, [Tay93, $\S 2])$.

In particular, we have $\nu \circ \bar{\rho} \neq 1$ by the assumption $p-1>\mathbf{w}$.

(2) A recent result of Ramakrishnan [Ram04] implies that, even without assuming $\left(R L I_{2}\right)$, for any $\pi$ occurring in the middle degree cohomohology of the Siegel threefold, the representation $\rho_{\pi, p}$ is absolutely irreducible, provided $(p+1) / 2>\mathbf{w}_{\pi}=\mathbf{w}$.

Let $A$ be an $\mathcal{O}$-algebra.

Definition 1.1. (1) A representation $\rho: \Gamma \rightarrow G(A)$ is called ordinary at $p$ of weight $\underline{i}=\left(i_{0}, i_{1}, i_{2}, i_{3}\right)$ with $i_{0} \leqslant i_{1} \leqslant i_{2} \leqslant i_{3}$ (integers in $\mathbb{Z}$ ) if there exists a symplectic basis such that

$$
\rho \mid I_{p}=\left(\begin{array}{cccc}
\epsilon^{-i_{0}} & * & * & * \\
0 & \epsilon^{-i_{1}} & * & * \\
0 & 0 & \epsilon^{-i_{2}} & * \\
0 & 0 & 0 & \epsilon^{-i_{3}}
\end{array}\right) .
$$

(2) It is called nearly ordinary if for any decomposition group $D_{p}$ at $p$, the flag associated to a suitable symplectic basis is $D_{p^{-}}$-stable.

Let $D_{p}$ be a decomposition group at $p$. For any $\alpha \in \mathcal{O}^{\times}$, let $\xi(\alpha): D_{p} \rightarrow \mathcal{O}^{\times}$be the unramified character of $D_{p}$ sending a geometric Frobenius to $\alpha$. Urban has shown [Urb05] (see also [GT05, Proposition 4.3.4]) the following result.

Theorem 1. Let $\pi$ be cuspidal, cohomological of weight $a \geqslant b \geqslant 0$ unramified at $p$ ordinary at $p$ in the automorphic sense, that is, the roots $\alpha_{p}, \beta_{p}, \gamma_{p}, \delta_{p}$ of the Hecke polynomial $P_{\pi, p}$ have $p$-adic ordinals $0, b+1, a+2, \mathbf{w}$, respectively. Then, if $\pi$ is stable at $\infty$, the restriction of $\rho_{\pi, p}$ to $D_{p}$ is conjugated (in $\mathrm{GL}_{4}$ ) to:

$$
\left(\begin{array}{cccc}
\xi\left(\alpha_{p}\right) & * & * & * \\
0 & \xi\left(p^{-b-1} \beta_{p}\right) \epsilon^{-b-1} & * & * \\
0 & 0 & \xi\left(p^{-a-2} \gamma_{p}\right) \epsilon^{-a-2} & * \\
0 & 0 & 0 & \xi\left(p^{-\mathbf{w}} \delta_{p}\right) \epsilon^{-\mathbf{w}}
\end{array}\right)
$$

We need to introduce the following strict ordinarity condition, which will play a role in the main theorem.

Condition (SO). We say that $(\pi, p)$ is strictly ordinary if $\pi$ is unramified ordinary at $p$ and that $a>b>0$, or $b=0$ and $\alpha_{p} \not \equiv p^{-1} \beta_{p}(\bmod \varpi)$, or $a=b$ and $\beta_{p} \not \equiv p^{-1} \gamma_{p}\left(\bmod p^{b+1} \varpi\right)$.

Remark. It follows from [Sch05, Table 2], that if $\pi$ is unramified ordinary at $p$, then $a>b>0$, or $b=0$ and $\alpha_{p} \neq p^{-1} \beta_{p}$, or $a=b$ and $\beta_{p} \neq p^{-1} \gamma_{p}$.

Corollary 1.2. Condition (SO) implies $\left(\mathrm{Reg}^{*}\right)$ for the representation $\bar{\rho}$.

The proof follows easily from Theorem 1. 


\section{NEARLY ORDINARY RANK FOUR GALOIS REPRESENTATIONS}

Let $S$ be the set of prime factors of $N$. It is now time to specify the local components $K_{\ell}(\ell \in S)$ of the level group $K \subset G(\widehat{\mathbb{Z}})$ that we allow. Let $N=N_{1} M_{1}$ with $N_{1}$ squarefree and $M_{1}$ divisible by $\prod_{\ell \mid M_{1}} \ell^{2}$ relatively prime to $N_{1}$. Then, we have the following.

- For $\ell$ dividing $N_{1}$, the group $K_{\ell}$ must be one of the following:

(1) the Klingen parahoric, consisting in matrices in $G\left(\mathbb{Z}_{\ell}\right)$ congruent $\bmod \ell$ to

$$
\left(\begin{array}{llll}
a & * & * & * \\
0 & * & * & * \\
0 & * & * & * \\
0 & 0 & 0 & *
\end{array}\right)
$$

$(1)^{+}$the strict Klingen parahoric, consisting of matrices as above with the left uppermost coefficient $a=1$;

(2) the Iwahori, consisting of matrices congruent $\bmod \ell$ to an element of $B(\mathbb{Z} / \ell \mathbb{Z})$.

- For $\ell$ dividing $M_{1}$ :

(3) $K_{\ell}$ is arbitrary.

Definition 1.3. We say that $(\pi, p)$ is $S$-good if for any $\ell \in S, K_{\ell}$ is as above, and we have the following.

- For $\ell$ dividing $M_{1}, \bar{\rho}$ restricted to $I_{\ell}$ is absolutely irreducible.

- For $\ell$ dividing $N_{1}$, we have the following.

- In case (1), $\operatorname{dim} \pi_{\ell}^{K}=1$ and $\bar{\rho} \mid I_{\ell}$ satisfies $\mathrm{UN}_{2,2}$ (unipotent with two Jordan blocks): the image of $I_{\ell}$ by $\bar{\rho}$ is generated by a conjugate of

$$
\epsilon_{2}=\left(\begin{array}{rrrr}
1 & 1 & 0 & 0 \\
0 & 1 & 0 & 0 \\
0 & 0 & 1 & -1 \\
0 & 0 & 0 & 1
\end{array}\right)
$$

- In case $(1)^{+}, \pi^{K_{\ell}, \chi} \neq 0$, for some non-trivial character $\chi=\chi_{\pi}$ of $(\mathbb{Z} / \ell \mathbb{Z})^{\times}$and $\left.\bar{\rho}\right|_{I_{\ell}}$ is 'peu ramifiée' $\mathrm{PR}_{2}$ :

$$
\left.\bar{\rho}\right|_{I_{\ell}} \cong\left(\begin{array}{cccc}
1 & 0 & 0 & 0 \\
0 & 1 & 0 & 0 \\
0 & 0 & \bar{\chi} & 0 \\
0 & 0 & 0 & \bar{\chi}
\end{array}\right)
$$

where $\bar{\chi}$, the reduction mod $\varpi$ of the Galois avatar of $\chi_{\pi}$, is non-trivial, and where the unramified and the $\bar{\chi}$-variant planes for $I_{\ell}$ are totally isotropic.

- In case $(2), \operatorname{dim} \pi^{K_{\ell}}=1$ and the image of $I_{\ell}$ by $\bar{\rho}$ is generated by a conjugate of the standard regular unipotent

$$
\epsilon=\left(\begin{array}{rrrr}
1 & 1 & 0 & 0 \\
0 & 1 & 1 & 0 \\
0 & 0 & 1 & -1 \\
0 & 0 & 0 & 1
\end{array}\right) .
$$

Remark. Note that the matrix $\epsilon_{2}$ is conjugated in $G$ to

$$
\epsilon_{2}^{\prime}=\left(\begin{array}{llll}
1 & 0 & 0 & 1 \\
0 & 1 & 1 & 0 \\
0 & 0 & 1 & 0 \\
0 & 0 & 0 & 1
\end{array}\right)
$$




\section{J. Tilouine}

In fact there are exactly two classes of unipotent matrices of exponent 2 in $G\left(\overline{\mathbb{Q}}_{p}\right)$ : that of $\epsilon_{2}$ and that of

$$
\eta_{2}=\left(\begin{array}{llll}
1 & & & \\
& 1 & 1 & \\
& 0 & 1 & \\
& & & 1
\end{array}\right) .
$$

Remark. One could introduce a case $\left(2^{\prime}\right)$ for primes $\ell$ dividing $N_{1}$ such that $K_{\ell}$ is the Siegel parahoric, $\operatorname{dim} \pi^{K_{\ell}}=1$ and $\bar{\rho} \mid I_{\ell}$ satisfies $\mathrm{UN}_{1,2,1}$ (unipotent with three Jordan blocks): the image $I_{\ell}$ by $\bar{\rho}$ is generated by a conjugate of

$$
\epsilon_{2}=\left(\begin{array}{llll}
1 & 0 & 0 & 0 \\
0 & 1 & 1 & 0 \\
0 & 0 & 1 & 0 \\
0 & 0 & 0 & 1
\end{array}\right) .
$$

However, this case resists our analysis for the moment, so we need to exclude it.

In the sequel, we assume that $(\pi, p)$ is $S$-good.

Definition 1.4. We say that a deformation $\rho: \Gamma \rightarrow G(A)$ of $\bar{\rho}$ is $S$-minimal if the following hold.

(1) If $\ell$ divides $M_{1}, \rho\left(I_{\ell}\right) \cong \bar{\rho}\left(I_{\ell}\right)$ by reduction $\bmod \mathfrak{m}_{A}$.

(2) If $\ell$ is of type (1), $\rho\left(I_{\ell}\right)$ is generated by a conjugate of $\epsilon_{2}$.

(3) If $\ell$ is of type $(1)^{+}$,

$$
\left.\rho\right|_{I_{\ell}} \sim\left(\begin{array}{cccc}
1 & 0 & 0 & 0 \\
0 & 1 & 0 & 0 \\
0 & 0 & \chi_{\pi} & 0 \\
0 & 0 & 0 & \chi_{\pi}
\end{array}\right)
$$

for the fixed character of $\operatorname{Gal}\left(\mathbb{Q}_{\ell}\left(\zeta_{\ell}\right) / \mathbb{Q}_{\ell}\right)$ which is the Galois avatar of $\chi_{\pi}$.

(4) If $\ell$ is of type (2), $\rho\left(I_{\ell}\right)$ is generated by a conjugate of the standard regular unipotent $\epsilon$.

The conjectural compatibility between the global and the local Langlands correspondence at $\ell$ implies that the types above for $\rho_{\pi, p} \mid I_{\ell}$, for $\ell$ dividing $N_{1}$, are indeed correct (see the description of the Weil-Deligne representation associated to $\pi_{\ell}$ admitting an Iwahori fixed vector in [Sch05, Table 3]).

\section{Universal nearly ordinary objects}

\subsection{Deformation ring}

Let $\bar{\rho}=\rho_{\pi, p}(\bmod \varpi)$ for $(\pi, p)$ as above, satisfying $p-1>w=a+b+3, p$ prime to $N$, ordinarity and strict ordinarity, $\left(R L I_{2}\right)$ and $S$-goodness.

Let $\mathrm{CNL}_{\mathcal{O}}$ be the category of complete noetherian local $\mathcal{O}$-algebras whose residue field is $k$. For each object $A$ of $\mathrm{CNL}_{\mathcal{O}}$, let $G(A)^{+}$be the kernel of the reduction map $G(A) \rightarrow G(k)$. Fix a decomposition group $D_{p}$ at $p$ in $\Gamma$. Consider the deformation problem $\mathcal{F}$ of $\bar{\rho}$ sending an object $A$ of $\mathrm{CNL}_{\mathcal{O}}$ to the quotient of a set $E(A)$ of liftings $\rho: \Gamma \rightarrow G(A)$ of $\bar{\rho}$ by the equivalence relation of conjugation by elements of $G(A)^{+}$. The set $E(A)$ is the set of liftings $\rho$ such that:

(1) there exists $g \in G(A)$ such that $g \rho\left(D_{p}\right) g^{-1} \subset B(A)$, in such a way that the reduction of the representation of $D_{p}$ given by $g \bar{\rho} g^{-1}(\bmod N(A))$ modulo $\mathfrak{m}$ is exactly $\bar{\rho}(\bmod N(k))$;

(2) for each $\ell \in S, \rho \mid I_{\ell}$ is minimal. 


\section{NEARLY ORDINARY RANK FOUR GALOIS REPRESENTATIONS}

Proposition 2.1. This problem is representable by a universal pair $\left(R, \rho_{R}\right)$ consisting in a universal deformation ring and a universal representation whose conjugacy class is in $\mathcal{F}(R)$.

Proof. By Corollary 1.2, the assumption of strict ordinary implies (Reg) and (Reg*) for $\bar{\rho}$; in other words, $\bar{\rho}\left(D_{p}\right)$ is upper triangular (we assume for simplicity that the conjugating element $\bar{g}$ for $\bar{\rho}$ is one, as it is harmless in the proof), with diagonal characters $\bar{\chi}_{i}$ such that $\bar{\chi}_{i} \neq \bar{\chi}_{i+1}$. The condition at $p$ is therefore innocuous for the verification of the glueing condition in Schlessinger's criterion. More precisely, if $A_{1} \rightarrow A_{0}$ and $A_{2} \rightarrow A_{0}$ are two morphisms in $\mathrm{CNL}_{\mathcal{O}}$, the first being surjective, if two elements $g_{i} \in G\left(A_{i}\right)$ are such that the representations $g_{i} \rho_{i} g_{i}^{-1}$ of $D_{p}$ defined over the rings $A_{i}$ do agree when pushed down to $A_{0}$, then one can modify $g_{1}$ in such a way that $\left(g_{1}, g_{2}\right)$ belongs to the fiber product $A_{1} \times A_{0} A_{2}$.

Similarly, for $\ell \in S$, if $\ell$ divides $N_{1}$, in cases (1) and (2), the smoothness of the normalizers of unipotent elements shows again that for $g_{i} \in G\left(A_{i}\right)$ such that $g_{i} \rho_{i}\left(I_{\ell}\right) g_{i}^{-1}$ is generated by the various standard unipotents, one can modify $g_{1}$ in such a way that the pair $\left(g_{1}, g_{2}\right)$ belongs to the fiber product $A_{1} \times{ }_{A_{0}} A_{2}$. In case $(1)^{+}$, we use the smoothness of the centralizer of the group of matrices diagonal by blocks $\left(\begin{array}{cc}1_{2} & 0 \\ 0 & \chi_{\pi} \cdot 1_{2}\end{array}\right)$, namely, the Levi of the Siegel parabolic.

For $\ell$ dividing $M_{1}$, there is no condition (see [GT05]).

As explained in $\S 1.1$, there is a natural continuous group homomorphism $H \rightarrow R^{\times}$. Let us be more precise. Note first that here, by definition of the Hida group, we have $H=T\left(\mathbb{Z}_{p}\right.$ ) (or $T\left(\mathbb{Z}_{p}\right) /\{ \pm 1\}$ where $\{ \pm 1\}$ is embedded diagonally in the torus, if $\left.-1 \equiv 1(\bmod N)\right)$. We can define the universal character $T\left(\mathbb{Z}_{p}\right) \rightarrow R^{\times}$as follows.

We consider the universal representation $\rho_{R}: \Gamma \rightarrow \widehat{G}(R)$; by restriction to $D_{p}$ and reduction modulo the unipotent radical $\widehat{N}$ of $\widehat{B}$, we get a homomorphism $D_{p} \rightarrow \widehat{T}(R)$. It factors through the abelianization of $D_{p}$; its restriction to the inertia $I\left(\mathbb{Q}_{p}^{a b} / \mathbb{Q}_{p}\right)$ provides a continuous homomorphism $\phi$ from $I\left(\mathbb{Q}_{p}^{a b} / \mathbb{Q}_{p}\right)$ to $\widehat{T}(R)$. We identify $I\left(\mathbb{Q}_{p}^{a b} / \mathbb{Q}_{p}\right)$ to $\mathbb{Z}_{p}^{\times}$by the cyclotomic character to the exponent minus one $\epsilon^{-1}: I\left(\mathbb{Q}_{p}^{a b} / \mathbb{Q}_{p}\right) \rightarrow \mathbb{Z}_{p}^{\times}$. Recall $\widehat{T}(R)=X^{*}(T) \otimes R^{\times}$; we take as the basis of $X^{*}(T)$ the characters $t_{1}, t_{2}$ and $\nu$ sending $t=\operatorname{diag}\left(t_{1}, t_{2}, \nu t_{2}^{-1}, \nu t_{1}^{-1}\right)$ to the corresponding values. Then, we can write $\phi$ as a triple of $R$-valued characters of $\mathbb{Z}_{p}^{\times}\left(\phi_{1}, \phi_{2}, \phi_{\nu}\right)$. The dual basis of $\left\{t_{1}, t_{2}, \nu\right\}$ in $X_{*}(T)$ is still denoted by the same letters. Then, the character $T\left(\mathbb{Z}_{p}\right) \rightarrow R^{\times}$is given by the product

$$
t=\operatorname{diag}\left(t_{1}, t_{2}, \nu t_{2}^{-1}, \nu t_{1}^{-1}\right) \mapsto \phi_{1}\left(t_{1}\right) \phi_{2}\left(t_{2}\right) \phi_{\nu}(\nu)
$$

In order to relate $\phi$ to the characters $\chi_{i, R}$ and $\nu_{R}=\nu \circ \rho_{R}$ giving the diagonal of $\rho_{R}$ in $T(R)$, we need to explicit the spin isomorphism; we fix $\widehat{T}(R)=\left(R^{\times}\right)^{3}$ using the standard basis of $X^{*}(T)$ as above. Then, spin : $\widehat{T}(R)=\left(R^{\times}\right)^{3} \rightarrow T(R)$ becomes $\left(t_{1}, t_{2}, \nu\right) \mapsto \operatorname{diag}\left(\nu, t_{2} \nu, t_{1} \nu, t_{1} t_{2} \nu\right)$ and the homomorphism $D_{p} \rightarrow T(R)$ is given by $\operatorname{diag}\left(\chi_{1, R}, \chi_{2, R}, \nu_{R} \chi_{2, R}^{-1}, \nu_{R} \chi_{1, R}^{-1}\right)$.

Therefore we have to solve the equations

$$
\phi_{\nu} \circ \epsilon^{-1}=\chi_{1, R},\left(\phi_{\nu} \phi_{2}\right) \circ \epsilon^{-1}=\chi_{2, R} \quad \text { and } \quad\left(\phi_{\nu} \phi_{1}\right) \circ \epsilon^{-1}=\nu_{R} \chi_{2, R}^{-1} .
$$

Using the identification $\epsilon^{-1}: I\left(\mathbb{Q}_{p}^{a b} / \mathbb{Q}_{p}\right) \rightarrow \mathbb{Z}_{p}^{\times}$, this yields the formula for the universal character $T\left(\mathbb{Z}_{p}\right) \rightarrow R^{\times}:$

$$
t=\operatorname{diag}\left(t_{1}, t_{2}, \nu t_{2}^{-1}, \nu t_{1}^{-1}\right) \mapsto \nu_{R} \chi_{1, R}^{-1} \chi_{2, R}^{-1}\left(t_{1}\right) \chi_{2, R} \chi_{1, R}^{-1}\left(t_{2}\right) \chi_{1, R}(\nu) .
$$

In fact, because of the fact that we deal with Satake parameters instead of Langlands parameters, it will be necessary to consider a version of this morphism twisted by minus the character $\rho=(2,1 ; 0)$ of $T$ given as the half-sum of the positive roots of $(G, B, T)$. 


\section{J. Tilouine}

Definition 2.2. The twisted universal character $T\left(\mathbb{Z}_{p}\right) \rightarrow R^{\times}$associated to the universal nearly ordinary representation $\rho_{R}$ is

$$
t=\operatorname{diag}\left(t_{1}, t_{2}, \nu t_{2}^{-1}, \nu t_{1}^{-1}\right) \mapsto t_{1}^{-2} t_{2}^{-1} \cdot \nu_{R} \chi_{1, R}^{-1} \chi_{2, R}^{-1}\left(t_{1}\right) \chi_{2, R} \chi_{1, R}^{-1}\left(t_{2}\right) \chi_{1, R}(\nu) .
$$

It is called canonical in the sequel.

We endow $R$ with the structure of $\mathcal{O}[[H]]$-algebra defined by the canonical character.

\subsection{Cohomology module}

For each $r \geqslant 1$, let $I_{r}=\left\{g \in G\left(\mathbb{Z}_{p}\right) ; g\left(\bmod p^{r}\right) \in B\left(\mathbb{Z} / p^{r} \mathbb{Z}\right)\right\}$ be the Iwahori subgroup of level $p^{r}$ and $I_{r}^{+}$be its normal subgroup such that we have $I_{r} / I_{r}^{+} \cong T\left(\mathbb{Z} / p^{r} \mathbb{Z}\right)$ by $n^{-} t n^{+} \mapsto t\left(\bmod p^{r}\right)$ in the Iwahori decomposition. As before, $p$ is assumed to be prime to $N$ and such that $p-1>a+b+3$. Under this assumption, there is no ambiguity in the choice of an $\mathcal{O}$-lattice $G\left(\mathbb{Z}_{p}\right)$-stable $V_{a, b}(\mathcal{O})$ in $V_{a, b}(F)$ (see [MT02]). It defines a local system $V_{a, b}(F / \mathcal{O})=V_{a, b}(\mathcal{O}) \otimes F / \mathcal{O}$ over the Siegel varieties; we form $\widetilde{\mathcal{V}}=\lim _{\longrightarrow} H^{3}\left(S_{K^{p} \times I_{r}^{+}}, V_{a, b}(F / \mathcal{O})\right)$ where the inductive limit is taken with respect to $\phi_{L, L^{\prime}}^{*}$ for the transition maps $\phi_{L, L^{\prime}}: S_{L} \rightarrow S_{L^{\prime}}$. For any $\mathcal{O}$-module $A$, let $A^{*}=\operatorname{Hom}_{\mathcal{O}}(A, F / \mathcal{O})$ be the $\mathcal{O}$-Pontryagin dual of $A$. We put $\widetilde{\mathbf{V}}=\widetilde{\mathcal{V}}^{*}$.

The compact $\mathcal{O}$-module $\widetilde{\mathbf{V}}$ carries a natural structure of $T\left(\mathbb{Z}_{p}\right)$-module given by normal action of $\lim _{r} I_{r} / I_{r}^{+} ;$in the case $-1 \equiv 1(\bmod N)$, it factors through $H=T\left(\mathbb{Z}_{p}\right) /\{ \pm 1\}$, because the action of $T\left(\mathbb{Z}_{p}\right)$ restricted to the center $Z\left(\mathbb{Z}_{p}\right)$ is global hence factors modulo $Z(\mathbb{Z})=\{ \pm 1\}$. Otherwise, the action of $T\left(\mathbb{Z}_{p}\right)$ on $\tilde{\mathbf{V}}$ is faithful. Note that this action depends on $(a, b)$. For any $t \in T\left(\mathbb{Z}_{p}\right)$, let us write $[t]^{a, b}$ for this action on $\widetilde{\mathbf{V}}$. We modify it to make it of weight 0 by posing $[t]=t_{1}^{a} t_{2}^{b}[t]^{a, b}$, in such a way that $H^{3}\left(S_{K^{p} \times I_{r}^{+}}, V_{a, b}(F / \mathcal{O})\right)$ maps naturally to the submodule of $\widetilde{\mathcal{V}}$ on which $[t]=t_{1}^{a} t_{2}^{b}$ for any $t \in \operatorname{Ker}\left(T\left(\mathbb{Z}_{p}\right) \rightarrow T\left(\mathbb{Z} / p^{r} \mathbb{Z}\right)\right)$ by the inductive limit of transition maps. Let $\mathbb{T}=T\left(\mathbb{Z}_{p}\right)$. This is the action $\mathbb{T} \rightarrow \operatorname{End}_{\mathcal{O}}(\widetilde{\mathbf{V}}), t \mapsto[t]$ that we fix; it gives rise to a natural structure of $\mathcal{O}[[H]]$-module on $\widetilde{\mathbf{V}}$.

Let $\mathbb{T}^{+}$be the kernel of the reduction map $\mathbb{T}=T\left(\mathbb{Z}_{p}\right) \rightarrow T(\mathbb{Z} / p \mathbb{Z})$, it is also the pro-p-Sylow of the Hida group. Let $\Lambda=\mathcal{O}\left[\left[\mathbb{T}^{+}\right]\right]$. We call this algebra the Hida-Iwasawa algebra. It is a regular ring of relative dimension 3 over $\mathcal{O}$.

We are concerned with certain prime ideals of $\Lambda$, suited for the $p$-adic interpolation.

Definition 2.3. For any triple $\left(a^{\prime}, b^{\prime} ; c^{\prime}\right) \in \mathbb{Z}^{3}$ such that $a^{\prime} \geqslant b^{\prime} \geqslant 0$ and $c^{\prime} \equiv a^{\prime}+b^{\prime}(\bmod 2)$, we denote by $P_{a^{\prime}, b^{\prime} ; c^{\prime}}$ the ideal of $\Lambda$ defined as the kernel of the morphism $\Lambda \rightarrow \mathcal{O}$ associated to the character $t \in \mathbb{T}^{+} \mapsto t_{1}^{a^{\prime}} t_{2}^{b^{\prime}} \nu^{\left(c^{\prime}-a^{\prime}-b^{\prime}\right) / 2}$, where we write as usual an element $t \in T$ as $t=$ $\operatorname{diag}\left(t_{1}, t_{2}, \nu t_{2}^{-1}, \nu t_{1}^{-1}\right)$. We call $P_{a^{\prime}, b^{\prime} ; c^{\prime}}$ an admissible algebraic point of $\Lambda$.

These ideals are dimension 1 , generated by regular sequences.

Recall also that we assume $(\pi, p)$ to be ordinary at $p$; we order the roots of the Hecke polynomial $P_{\pi, p}$ by increasing ordinal: $\operatorname{ord}_{p}(\alpha)=0, \operatorname{ord}_{p}(\beta)=b+1, \operatorname{ord}_{p}(\gamma)=a+2, \operatorname{ord}_{p}(\delta)=a+b+3$. Let us consider the commutative subalgebra $\mathbb{Z}\left[U_{p, 1}, p^{-b} U_{p, 2}\right]$ of the abstract Hecke algebra $\mathcal{H}_{I_{r}^{+}}$generated over $\mathbb{Z}$ by the two operators $U_{p, 1}$ and $p^{-b} U_{p, 2}$ where

$$
U_{p, 1}=I_{r}^{+}\left(\begin{array}{cccc}
1 & & & \\
& 1 & & \\
& & p & \\
& & & p
\end{array}\right) I_{r}^{+} \quad \text { and } \quad U_{p, 2}=I_{r}^{+}\left(\begin{array}{cccc}
1 & & \\
& p & & \\
& & p & \\
& & p^{2}
\end{array}\right) I_{r}^{+} .
$$

Let $\mathfrak{m}^{p}$ be the maximal ideal of $\mathcal{H}^{N p}$ generated by $\left(\varpi, T-\theta_{\pi}(T) ; T \in \mathcal{H}^{N p}\right)$; we define a maximal ideal in the Hecke algebra $\mathcal{H}^{N p} \otimes \mathbb{Z}\left[U_{p, 1}, p^{-b} U_{p, 2}\right]$ by $\mathfrak{m}=\mathfrak{m}^{p} \otimes 1+1 \otimes\left(\varpi, U_{p, 1}-\alpha, p^{-b} U_{p, 2}-p^{-b-1} \alpha \beta\right)$. We put $\mathbf{V}=\widetilde{\mathbf{V}}_{\mathfrak{m}}$; it is the Pontryagin dual of $\lim _{\longrightarrow} H^{3}\left(S_{K^{p} \times I_{r}^{+}}, V_{a, b}(F / \mathcal{O})\right)_{\mathfrak{m}}$. 


\section{NEARLY ORDINARY RANK FOUR GALOIS REPRESENTATIONS}

Recall a result of [MT02, Theorem 9]. Let $\left(a^{\prime}, b^{\prime} ; c^{\prime}\right)$ be a triple of integers such that $a^{\prime} \geqslant b^{\prime} \geqslant 0$, $c^{\prime} \equiv a^{\prime}+b^{\prime}(\bmod 2)$ and $V_{a^{\prime}, b^{\prime} ; c^{\prime}}$ be the Weyl irreducible representation of $G$ over $F$ of highest weight $\left(a^{\prime}, b^{\prime}\right)$ and central character $z \mapsto z^{c^{\prime}}$. With this notation, the representation that we denoted by $V_{a, b}$ should be denoted as $V_{a, b ; a+b}$. We will omit this extra index in the sequel.

Let $\omega: \mathbb{Z}_{p}^{\times} \rightarrow \mu_{p-1}$ be the projection to the finite-order subgroup of $\mathbb{Z}_{p}^{\times}$and for any triple $(x, y, z)$ of integers modulo $p-1$, let

$$
\omega_{1}^{x} \otimes \omega_{2}^{y} \otimes \omega_{\nu}^{z}: \operatorname{diag}\left(t_{1}, t_{2}, \nu t_{1}^{-1}, \nu t_{2}^{-1}\right) \mapsto \omega\left(t_{1}\right)^{x} \omega\left(t_{2}\right)^{y} \omega(\nu)^{z} .
$$

One then has the control theorem.

Theorem 2. (1) $\mathbf{V}$ does not depend on $(a, b)$ (provided $a \geqslant b \geqslant 0$ ).

(2) Assume $p-1>\mathbf{w}, p$ prime to $N,\left(R L I_{2}\right)$ and $(\pi, p)$ ordinary; then $\mathbf{V}$ is finite free over $\Lambda$.

(3) For any admissible algebraic prime $P_{a^{\prime}, b^{\prime} ; c^{\prime}}$ of $\Lambda$, there is a canonical isomorphism $\mathbf{V} / P_{a^{\prime}, b^{\prime}, c^{\prime}} \mathbf{V} \cong$ $\mathbf{V}_{a^{\prime}, b^{\prime}, c^{\prime}}$ where $\mathbf{V}_{a^{\prime}, b^{\prime}, c^{\prime}}$ is the Pontryagin dual of

$$
H^{3}\left(S_{K^{p} \times I_{1}}, V_{a^{\prime}, b^{\prime} ; c^{\prime}}(F / \mathcal{O}) \otimes \omega_{1}^{a-a^{\prime}} \otimes \omega_{2}^{b-b^{\prime}} \otimes \omega_{\nu}^{-c^{\prime}}\right)_{\mathfrak{m}}
$$

\subsection{Hecke algebra}

We then define the Hecke algebra $\mathbf{T}$ as the $\Lambda$-algebra generated by the image of $\mathcal{H}^{N p}$ in $\operatorname{End} \mathcal{O}(\mathbf{V})$. For any dominant weight $\left(a^{\prime}, b^{\prime} ; c^{\prime}\right)$ as above, let $\mathbf{T}_{a^{\prime}, b^{\prime} ; c^{\prime}}$ be the $\mathcal{O}$-algebra generated by $\mathcal{H}^{N p}$ in $\operatorname{End}_{\mathcal{O}} \mathbf{V}_{a^{\prime}, b^{\prime} ; c^{\prime}}$.

Corollary 2.4. For any algebraic prime $P_{a^{\prime}, b^{\prime} ; c^{\prime}}$, the canonical $\mathcal{O}$-algebra homomorphism $\mathbf{T} / P_{a^{\prime}, b^{\prime} ; c^{\prime}} \mathbf{T} \rightarrow \mathbf{T}_{a^{\prime}, b^{\prime} ; c^{\prime}}$ is surjective and factors into an isomorphism modulo the nilradical of the source.

\subsection{Galois representation}

Theorem 3. There exists a deformation $\rho_{\mathbf{T}} \in \mathcal{F}(\mathbf{T})$ of $\bar{\rho}$ characterized as follows. For any dominant weight $\left(a^{\prime}, b^{\prime} ; c^{\prime}\right)$ as above, for any cuspidal representation $\pi^{\prime}$ occurring in $H^{3}\left(S_{K^{p} \times I_{1}}, V_{a, b}(\mathbb{C})\right)_{\mathfrak{m}}$, let $\Theta_{\pi^{\prime}}: \mathbf{T} \rightarrow \overline{\mathbb{Q}}_{p}$ be the composition of the specialization morphism $\mathbf{T} \rightarrow \mathbf{T}_{a^{\prime}, b^{\prime} ; c^{\prime}}$ with the character $\theta_{\pi^{\prime}}$. Then, $\Theta_{\pi^{\prime}}$ sends $\rho_{\mathbf{T}}$ to $\rho_{\pi^{\prime}, p}$ (up to conjugation in $G\left(\overline{\mathbb{Q}}_{p}\right)$ ).

Proof. The existence of $\rho_{\mathbf{T}}$ follows from standard arguments on pseudo-representations (its symplecticity, assuming $\left(R L I_{2}\right)$, is proven in $\left.[G T 05, \S 3]\right)$. Let us check the local conditions at $p$ and $S$. For the prime $p$, we recall first the following result (see [TU99, Proposition 3.2]) which allows us to remove $p$ from the level for regular weights. For any regular dominant weight $\left(a^{\prime}, b, ; c^{\prime}\right)$ (i.e. $a^{\prime}>b^{\prime}>0$ and $\left.c^{\prime} \equiv a^{\prime}+b^{\prime}(\bmod 2)\right)$, there is a Galois-equivariant isomorphism

$$
H^{3}\left(S_{K^{p} \times I_{1}}, V_{a^{\prime}, b^{\prime} ; c^{\prime}}(F / \mathcal{O})\right)_{\mathfrak{m}} \cong H^{3}\left(S_{K^{p} \times G\left(\mathbb{Z}_{p}\right)}, V_{a^{\prime}, b^{\prime} ; c^{\prime}}(F / \mathcal{O})\right)_{\mathfrak{m}} .
$$

Thus, one sees, by applying Urban's theorem to regular dominant weights $\left(a^{\prime}, b^{\prime} ; c^{\prime}\right)$ such that $a^{\prime} \equiv a$ $(\bmod p-1), b^{\prime} \equiv b(\bmod p-1)$ and $c^{\prime} \equiv a+b(\bmod p-1)$, that

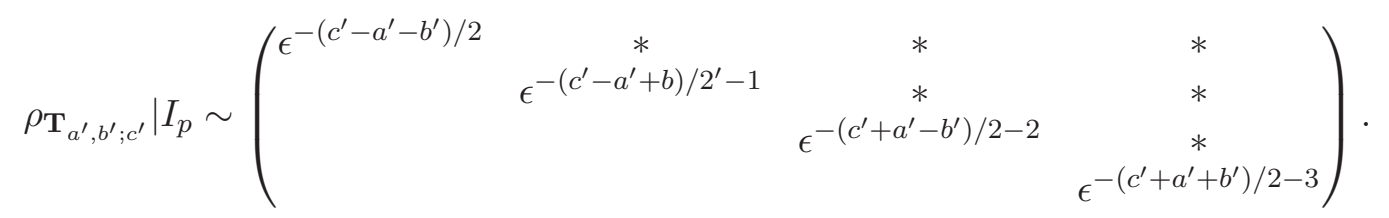

For any element $x \in 1+p \mathbb{Z}_{p}$, we define elements $[x]_{*} \in \Lambda$, for $*=1,2, \nu$ or $c$, as follows: first apply the cocharacter $\xi_{*}: 1+p \mathbb{Z}_{p} \rightarrow \mathbb{T}^{+}$, then compose with the canonical inclusion $\mathbb{T}^{+} \rightarrow \Lambda$; where $\xi_{1}: x \mapsto \operatorname{diag}\left(x, 1,1, x^{-1}\right), \xi_{2}: x \mapsto \operatorname{diag}\left(1, x, x^{-1}, 1\right), \xi_{\nu}: x \mapsto \operatorname{diag}(1,1, x, x)$ and $\xi_{c}=i: x \mapsto$ $\operatorname{diag}(x, x, x, x)$. We shall compose these maps with the diamond operation $\Lambda \rightarrow \mathbf{T}$ so that we view 


\section{J. Tilouine}

the elements $[x]_{*}$ as sitting in $\mathbf{T}$ without further notice. For any element $t=\operatorname{diag}\left(x, y, z x^{-1}, z y^{-1}\right)$ of $\mathbb{T}^{+}$, the diamond operator $[t]$ is equal to the product $[x]_{1}[y]_{2}[z]_{\nu}$.

Recall that we use the character $\epsilon^{-1}$ to identify the inertia subgroup $I\left(\mathbb{Q}_{p}^{a b} / \mathbb{Q}_{p}\right)$ to $\mathbb{Z}_{p}^{\times}$. Let us decompose the $p$-adic cyclotomic character into a (prime-to- $p$ ) finite-order character and a pro- $p$ order character, $\epsilon=\omega \epsilon_{1}$. Put $\epsilon_{1}^{-1}=\kappa$.

By Zariski-density of the set of admissible algebraic primes in Spec $\Lambda$, it follows from Urban's theorem and the control theorem (Theorem 2) that (see [GT05, Proposition 4.3.4]) the restriction of $\rho_{\mathbf{T}}$ to a decomposition group $D_{p}$ at $p$ leaves stable a flag

$$
0 \subset \mathrm{Fil}^{(3)} \subset \mathrm{Fil}^{(2)} \subset \mathrm{Fil}^{(1)} \subset \mathrm{Fil}^{(0)}=\mathbf{T}^{4},
$$

with $\mathbf{T}$-free rank one graded quotients $\operatorname{gr}^{(i)}$ with the action of $I_{p}$ given, respectively, by:

- on $\operatorname{gr}^{(3)},[\kappa]_{1}^{-1 / 2}[\kappa]_{2}^{-1 / 2}[\kappa]_{c}^{1 / 2}$;

- on $\operatorname{gr}^{(2)}, \omega^{-b-1} \kappa[\kappa]_{1}^{-1 / 2}[\kappa]_{2}^{1 / 2}[\kappa]_{c}^{1 / 2}$;

- on $\operatorname{gr}^{(1)}, \omega^{-a-2} \kappa^{2}[\kappa]_{1}^{1 / 2}[\kappa]_{2}^{-1 / 2}[\kappa]_{c}^{1 / 2}$;

- on $\operatorname{gr}^{(0)}, \omega^{-a-b-3} \kappa^{3}[\kappa]_{1}^{-1 / 2}[\kappa]_{2}^{-1 / 2}[\kappa]_{c}^{1 / 2}$.

This shows, in particular, the near ordinarity of $\rho_{\mathbf{T}}$ at $p$.

For each $\ell \in S$, recall that we have fixed in $\S 1.2$ the local factors $K_{\ell}$ of our level group $K$ according to the form of the local Galois representation $\bar{\rho}$ at $\ell$. With these choices, it was proven in $[\mathrm{GT} 05, \S 9.2]$, that for any regular dominant weight $\left(a^{\prime}, b^{\prime} ; c^{\prime}\right)$, the representation $\rho_{\mathbf{T}_{a^{\prime}, b^{\prime} ; c^{\prime}}}$ does satisfy the minimality condition at $\ell$; the same for $\rho_{\mathbf{T}}$ follows by the inverse limit.

Corollary 2.5. There exists a surjective $\Lambda$-linear local rings homomorphism $\phi: R \rightarrow \mathbf{T}$, characterized by the property that the image of $\rho_{R}$ by $\phi$ is conjugate to $\rho_{\mathbf{T}}$ in $G(\mathbf{T})$.

The existence comes from the universal property of $R$; the surjectivity is classical (see [GT05, Proposition 4.3.8] for instance). The $\Lambda$-linearity results from the above description of $\rho_{\mathbf{T}} \mid I_{p}$. More precisely, we see that, if we write

$$
\operatorname{diag}\left(\chi_{1, \mathbf{T}}, \chi_{2, \mathbf{T}}, \nu_{\mathbf{T}} \chi_{2, \mathbf{T}}^{-1}, \nu_{\mathbf{T}} \chi_{1, \mathbf{T}}^{-1}\right)
$$

for the element of $T(\mathbf{T})$ given by $\rho_{\mathbf{T}} \mid D_{p} \bmod N(\mathbf{T})$, we have (in the notation of the previous section)

$$
\begin{gathered}
\chi_{1, \mathbf{T}}(\kappa)=[\kappa]_{1}^{-1 / 2}[\kappa]_{2}^{-1 / 2}[\kappa]_{c}^{1 / 2}=[\kappa]_{\nu}, \\
\chi_{2, \mathbf{T}}(\kappa)=\kappa[\kappa]_{1}^{-1 / 2}[\kappa]_{2}^{1 / 2}[\kappa]_{c}^{1 / 2}, \\
\nu_{\mathbf{T}} \chi_{2, \mathbf{T}}^{-1}(\kappa)=\kappa^{2}[\kappa]_{1}^{1 / 2}[\kappa]_{2}^{-1 / 2}[\kappa]_{c}^{1 / 2} .
\end{gathered}
$$

Therefore, a simple calculation implies that the image by $\phi$ of the canonical character $T\left(\mathbb{Z}_{p}\right) \rightarrow$ $R^{\times}$is

$$
t=\operatorname{diag}\left(t_{1}, t_{2}, \nu t_{2}^{-1}, \nu t_{1}^{-1}\right) \mapsto\left[t_{1}\right]_{1}\left[t_{2}\right]_{2}[\nu]_{\nu}=[t] .
$$

Note that the presence in the definition of the canonical character of a twist by the character $-\rho$, half-sum of the positive roots of $(G, B, T)$ is exactly what is needed to compensate for the occurrence of a factor $t^{\rho}=t_{1}^{2} t_{1}$ in this calculation.

\section{An $R=T$ theorem}

The main theorem of this paper is the following. 


\section{NEARLY ORDINARY RANK FOUR GALOIS REPRESENTATIONS}

Theorem 4. Assume that we are given a pair $(\pi, p)$ satisfying the assumptions of [GT05, Theorem 2.2.2]. If, moreover, it is $S$-good (see [GT05, Definition 1.2.3]) and satisfies Condition (SO), then the homomorphism $\phi: R \rightarrow \mathbf{T}$ is an isomorphism of $\Lambda$-algebras, the rings $R$ and $\mathbf{T}$ are finite flat over $\Lambda$; they are local complete intersections and $\mathbf{V}$ is free as a $\mathbf{T}$-module. Moreover, for any (not necessarily regular) dominant weight $\left(a^{\prime}, b^{\prime} ; c^{\prime}\right)$, one has an exact control theorem $\mathbf{T} / P_{a^{\prime}, b^{\prime} ; c^{\prime}} \mathbf{T} \cong \mathbf{T}_{a^{\prime}, b^{\prime} ; c^{\prime}}$.

The proof consists of reducing the diagram $\phi: R \rightarrow \mathbf{T} \hookrightarrow \operatorname{End}_{\Lambda}(\mathbf{V})$ modulo the prime $P=$ $P_{a, b ; a+b}$ of $\Lambda$ and applying [GT05, Main Theorem 4.3.9].

Before doing this, we need some preliminary facts.

\subsection{Preliminaries on ordinary Iwahori level cohomology}

Let $\pi_{p}^{\prime}$ be a smooth irreducible representation of $G\left(\mathbb{Q}_{p}\right)$ that is unramified. Then, one knows that $\left(\pi_{p}^{\prime}\right)^{I_{1}}$ is an eight-dimensional $\mathbb{C}$-space with an action of the Hecke algebra for the Iwahori subgroup. Let $\alpha_{\pi^{\prime}}, \beta_{\pi^{\prime}}, \gamma_{\pi^{\prime}}$ and $\delta_{\pi^{\prime}}$ be its Satake parameters. Recall that $\rho=(2,1)$ denotes the half-sum of the positive roots of $(G, B, T)$.

Lemma 3.1. (1) The operators $U_{p, 1}$ and $p U_{p, 2}$ acting on this space can be simultaneously diagonalized with the following pairs of eigenvalues:

$$
\begin{aligned}
& \left(\alpha_{\pi^{\prime}}, \alpha_{\pi^{\prime}} \beta_{\pi^{\prime}}\right),\left(\alpha_{\pi^{\prime}}, \alpha_{\pi^{\prime}} \gamma_{\pi^{\prime}}\right),\left(\beta_{\pi^{\prime}}, \alpha_{\pi^{\prime}} \beta_{\pi^{\prime}}\right),\left(\beta_{\pi^{\prime}}, \delta_{\pi^{\prime}} \beta_{\pi^{\prime}}\right), \\
& \left(\gamma_{\pi^{\prime}}, \alpha_{\pi^{\prime}} \gamma_{\pi^{\prime}}\right),\left(\gamma_{\pi^{\prime}}, \delta_{\pi^{\prime}} \gamma_{\pi^{\prime}}\right),\left(\delta_{\pi^{\prime}}, \beta_{\pi^{\prime}} \delta_{\pi^{\prime}}\right),\left(\delta_{\pi^{\prime}}, \beta_{\pi^{\prime}} \delta_{\pi^{\prime}}\right) .
\end{aligned}
$$

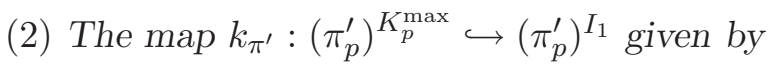

$$
\left(U_{p, 1}-\beta_{\pi^{\prime}}\right)\left(U_{p, 1}-\gamma_{\pi^{\prime}}\right)\left(U_{p, 1}-\delta_{\pi^{\prime}}\right) \cdot\left(p U_{p, 2}-\alpha_{\pi^{\prime}} \gamma_{\pi^{\prime}}\right)\left(p U_{p, 2}-\beta_{\pi^{\prime}} \delta_{\pi^{\prime}}\right)\left(p U_{p, 2}-\gamma_{\pi^{\prime}} \delta_{\pi^{\prime}}\right)
$$

defines an isomorphism from the one-dimensional left-hand side vector space to the subspace of the right-hand side vector space annihilated by $U_{p, 1}-\alpha$ and $p U_{p, 2}-\alpha_{\pi^{\prime}} \beta_{\pi^{\prime}}$.

Proof. Let $\chi$ be a character such that $\pi_{p}^{\prime}=\operatorname{Ind}_{B}^{G} \chi$ (non-normalized induction). We fix the ordering of the Satake parameters in such a way that $\chi(1,1, p, p)=\alpha_{\pi^{\prime}}, \chi(1, p, 1, p)=\beta_{\pi^{\prime}}, \chi(p, 1, p, 1)=\gamma_{\pi^{\prime}}$, $\chi(p, p, 1,1)=\delta_{\pi^{\prime}}$. We consider the isomorphism to the Jacquet module:

$$
\mathcal{J}:\left(\pi_{p}^{\prime}\right)^{I_{1}} \cong \bigoplus_{w \in W_{G}} \mathbb{C} e^{-\rho}\left(e^{\rho} \chi\right)^{w} .
$$

It transforms the action of the Iwahori-Hecke subalgebra generated by $U_{p, i}(i=1,2)$ on the left-hand side into the natural $T\left(\mathbb{Q}_{p}\right)$-action on the right-hand side (see [Cas, Theorem 3.3.3]).

(1) An easy calculation gives the action of the elements $\operatorname{diag}(1,1, p, p)$ respectively $\operatorname{diag}\left(1, p, p, p^{2}\right)$ of $T\left(\mathbb{Q}_{p}\right)$ on the right-hand side. It provides the pairs of eigenvalues of the lemma.

(2) For the second statement, one observes that the inverse image of the line $\mathbb{C} \chi$ by the isomorphism $\mathcal{J}$ is the subspace annihilated by $U_{p, 1}-\alpha$ and $p U_{p, 2}-\alpha_{\pi^{\prime}} \beta_{\pi^{\prime}}$; moreover, the calculations above show that the line $\mathbb{C} \chi$ is the image of the endomorphism $\mathcal{J} \circ k_{\pi^{\prime}} \circ \mathcal{J}^{-1}$.

Let $\mathfrak{m}^{\prime}$ be the maximal ideal of $\mathcal{H}^{N}=\mathcal{H}^{N p} \otimes \mathcal{H}_{p}$ defined as

$$
\mathfrak{m}^{\prime}=\mathfrak{m}^{p} \otimes 1+1 \otimes\left(T_{p, 1}-\alpha, p^{-b} T_{p, 2}-\alpha \beta / p^{b+1}\right) .
$$

It follows from the main theorem of [MT02] that the cohomology module

$$
H^{\bullet}\left(S_{K^{p} \times G\left(\mathbb{Z}_{p}\right)}, V_{a, b}(F / \mathcal{O})\right)_{\mathfrak{m}^{\prime}}
$$

is cofree, concentrated in degree 3 and cuspidal. Actually, the main theorem of [MT02] concerned the bigger module

$$
e_{0} H^{\bullet}\left(S_{K^{p} \times G\left(\mathbb{Z}_{p}\right)}, V_{a, b}(F / \mathcal{O})\right)_{\mathfrak{m}^{p}}
$$




\section{J. Tilouine}

where $e_{0}$ is the Hida idempotent, associated to the product of the Hecke operators $T_{p, 1}$ and $p^{-b} T_{p, 2}$. The statement above is obtained a fortiori by a localization at $\left(\varpi, T_{p, 1}-\alpha, p^{-b} T_{p, 2}-\alpha \beta / p^{b+1}\right)$.

Let $\phi=\phi_{L, L^{\prime}}$ be the transition morphism for $L=K^{p} \times I_{1}$ and $L^{\prime}=K^{p} \times G\left(\mathbb{Z}_{p}\right)$. It is finite étale of prime-to- $p$ degree equal to $\left(G\left(\mathbb{F}_{p}\right): B\left(\mathbb{F}_{p}\right)\right)=\left(1+p+p^{2}+p^{3}\right)(1+p)$. Inspired by Lemma 3.1(1), we introduce the Hecke operator $h \in \mathcal{O}\left[U_{p, 1}, p^{-b} U_{p, 2}\right]$ given by $h=h_{1} h_{2}$ where

$$
h_{1}=\left(U_{p, 1}-\beta\right)\left(U_{p, 1}-\gamma\right)\left(U_{p, 1}-\delta\right),
$$

and

$$
h_{2}=\left(p^{-b} U_{p, 2}-p^{-b-1} \alpha \gamma\right)\left(p^{-b} U_{p, 2}-p^{-b-1} \beta \delta\right)\left(p^{-b} U_{p, 2}-p^{-b-1} \gamma \delta\right) .
$$

Let us consider the map

$$
\Psi=h \circ \phi^{*}: H^{3}\left(S_{K^{p} \times G\left(\mathbb{Z}_{p}\right)}, V_{a, b}(F / \mathcal{O})\right)_{\mathfrak{m}^{\prime}} \rightarrow H^{3}\left(S_{K^{p} \times I_{1}}, V_{a, b}(F / \mathcal{O})\right) .
$$

\section{Proposition 3.2.}

(1) The map $\Psi$ takes values in the $\mathfrak{m}$ localization of $H^{3}\left(S_{K^{p} \times I_{1}}, V_{a, b}(F / \mathcal{O})\right)$.

(2) If, moreover, $(\pi, p)$ is strictly ordinary, then $\Psi$ is an isomorphism onto $H^{3}\left(S_{K^{p} \times I_{1}}, V_{a, b}(F / \mathcal{O})\right)_{\mathfrak{m}}$.

Proof. (1) It is enough to check this in characteristic zero, because the cokernels of

$$
H^{3}\left(S_{K^{p} \times I_{1}}, V_{a, b}(F)\right)_{\mathfrak{m}} \rightarrow H^{3}\left(S_{K^{p} \times I_{1}}, V_{a, b}(F / \mathcal{O})\right)_{\mathfrak{m}}
$$

respectively

$$
H^{3}\left(S_{K^{p} \times G\left(\mathbb{Z}_{p}\right)}, V_{a, b}(F)\right)_{\mathfrak{m}^{\prime}} \rightarrow H^{3}\left(S_{K^{p} \times G\left(\mathbb{Z}_{p}\right)}, V_{a, b}(F / \mathcal{O})\right)_{\mathfrak{m}^{\prime}}
$$

both vanish (cf. [MT02, Theorem 9] and [MT02, Theorem 1], respectively).

For this, we consider a cuspidal representation $\pi^{\prime}$ occurring in

$$
e_{0} H^{3}\left(S_{K^{p} \times G\left(\mathbb{Z}_{p}\right)}, V_{a, b}(\mathbb{C})\right)_{\mathfrak{m}^{p}} .
$$

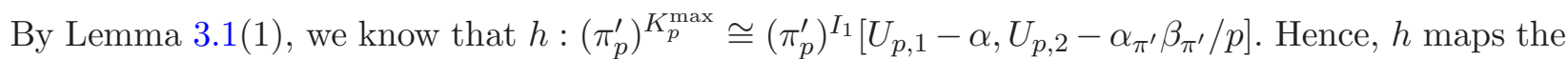
$\pi^{\prime}$-component of $H^{3}\left(S_{K^{p} \times G\left(\mathbb{Z}_{p}\right)}, V_{a, b}(F)\right)_{\mathfrak{m}^{p}}$ to that of $H^{3}\left(S_{K^{p} \times I_{1}}, V_{a, b}(F)\right)_{\mathfrak{m}}$, as desired.

(2) The surjectivity can again be checked in characteristic zero. If $a>b>0$, this is [TU99, Proposition 3.2]. We can therefore assume either that $b=0$ or $a=b$. Let $\pi^{\prime}$ be a cuspidal representation occurring in $H^{3}\left(S_{K^{p} \times I_{1}}, V_{a, b}(F / \mathcal{O})\right)_{\mathfrak{m}}$. Let $\alpha_{\pi^{\prime}}, \beta_{\pi^{\prime}}, \gamma_{\pi^{\prime}}, \delta_{\pi^{\prime}}$ be the Satake parameters of $\pi_{p}^{\prime}$ ordered according to their increasing $p$-ordinal; Schmidt's classification [Sch05, Table 3] of representations $\pi_{p}^{\prime}$ such that $\left(\pi_{p}^{\prime}\right)^{I_{1}} \neq 0$ shows that if $\left(\pi_{p}^{\prime}\right)^{K_{p}^{\max }}=0$, one must have either $\alpha_{\pi^{\prime}}=\beta_{\pi^{\prime}} / p$ or $\beta_{\pi^{\prime}}=\gamma_{\pi^{\prime}} / p$. By Lemma 3.1(1), these Satake parameters are also the eigenvalues of $U_{p, 1}$ on $\left(\pi_{p}^{\prime}\right)^{I_{1}}$. Therefore, there is a common eigenvector for the operators $U_{p, 1}$ and $p^{-b} U_{p, 2}$ with eigenvalues $\alpha_{\pi^{\prime}}$ and $\alpha_{\pi^{\prime}} \beta_{\pi^{\prime}} / p^{b+1}$, respectively, in $H^{3}\left(S_{K^{p} \times I_{1}}, V_{a, b}(F)\right)_{\mathfrak{m}}$. Since these operators are congruent to $\alpha$, respectively $\alpha \beta / p^{b+1} \bmod \mathfrak{m}$, we see that $\alpha_{\pi^{\prime}} \equiv \alpha(\bmod \varpi)$ and $\beta_{\pi^{\prime}} / p^{b+1} \equiv \beta / p^{b+1}(\bmod \varpi)$. Therefore, if $b=0$, the strict ordinarity condition $\alpha \not \equiv \beta / p(\bmod \varpi)$ precludes the condition $\alpha_{\pi^{\prime}}=\beta_{\pi^{\prime}} / p$. Similarly, let us exclude the possibility $\beta_{\pi^{\prime}}=\gamma_{\pi^{\prime}} / p$.

Let $\omega_{\pi^{\prime}}$ be the finite-order part of the central character of $\pi^{\prime}$ (it is a Dirichlet character defined modulo $N)$. Note that $\omega_{\pi^{\prime}} \equiv \omega_{\pi}(\bmod \varpi)$ because $\pi^{\prime}$ occurs in the $\mathfrak{m}$-component. Since $\beta_{\pi^{\prime}} \gamma_{\pi^{\prime}}=p^{a+b+3} \omega_{\pi^{\prime}}(p)$, this condition is equivalent to $\beta_{\pi^{\prime}} / p^{b+1}=p^{b+1} \omega_{\pi^{\prime}}(p) / \beta_{\pi^{\prime}}$. However, again, $\beta_{\pi^{\prime}} / p^{b+1} \equiv \beta / p^{b+1}(\bmod \varpi)$, and we know that if $a=b, \beta / p^{b+1} \not \equiv \gamma / p^{b+1}(\bmod \varpi)$, that is, $\beta / p^{b+1} \not \equiv p^{b+1} \omega_{\pi}(p) / \beta(\bmod \varpi)$. This contradiction precludes the possibility that $\beta_{\pi^{\prime}}=\gamma_{\pi^{\prime}} / p$.

Hence, $\left(\pi_{p}^{\prime}\right)^{K_{p}^{\max }} \neq 0$; the surjectivity follows using the fact that in the eight-dimensional space $\left(\pi_{p}^{\prime}\right)^{I_{1}}$, the subspace contributing to the $\mathfrak{m}$-localization of the cohomology is the line defined by the conditions $U_{p, 1}=\alpha_{\pi^{\prime}}$ and $U_{p, 2}=\alpha_{\pi^{\prime}} \beta_{\pi^{\prime}} / p$. Then, we apply Lemma 3.1(1) to conclude that $\Psi=h \circ \phi^{*}$ is surjective. 


\section{NEARLY ORDINARY RANK FOUR GALOIS REPRESENTATIONS}

Finally to show the injectivity, we produce an almost retraction of $\Psi$, that is, a homomorphism

$$
C: H^{3}\left(S_{K^{p} \times I_{1}}, V_{a, b}(F / \mathcal{O})\right)_{\mathfrak{m}} \rightarrow e_{0} H^{3}\left(S_{K^{p} \times G\left(\mathbb{Z}_{p}\right)}, V_{a, b}(F / \mathcal{O})\right)_{\mathfrak{m}^{p}}
$$

such that

$$
C \circ \Psi \text { is an automorphism of } e_{0} H^{3}\left(S_{K^{p} \times G\left(\mathbb{Z}_{p}\right)}, V_{a, b}(F / \mathcal{O})\right)_{\mathfrak{m}^{p}} .
$$

This is given by the Hecke correspondence

$$
C=\xi^{-1} \cdot \sum_{w \in W_{G}} I_{1} w I_{1}
$$

where

$$
\xi=(\alpha-\beta)(\alpha-\gamma)(\alpha-\delta) p^{-b+1}(\alpha \beta-\alpha \gamma) p^{-b+1}(\alpha \beta-\delta \beta) p^{-b+1}(\alpha \beta-\delta \gamma)
$$

then $(*)$ follows from the fact that $C \circ \Psi$ is congruent to $I d$ modulo $\mathfrak{m}$ by Lemma 3.1(1).

\subsection{End of the proof of Theorem 4}

Let us consider the diagram $\phi: R \rightarrow \mathbf{T} \hookrightarrow \operatorname{End}_{\Lambda}(\mathbf{V})$. We reduce it modulo the prime $P=P_{a, b ; a+b}$ of $\Lambda$.

We first see that $R / P R$ is the universal deformation ring $R_{a, b}$ classifying deformations $\rho$ of $\bar{\rho}$ that are $S$-minimal and such that

$$
\rho \mid I_{p} \sim\left(\begin{array}{cccc}
1 & & & \\
& \epsilon^{-b-1} & * & * \\
& & \epsilon^{-a-2} & * \\
& & & \epsilon^{-a-b-3}
\end{array}\right),
$$

that is, which they are ordinary at $p$.

This problem of deformation does not require the representations to be crystalline at $p$; however, under the assumption of strict ordinarity, we have shown in [GT05, Lemmas 10.4.3 and 10.4.4], using Galois cohomology calculations in order to bound the minimal number of generators of $R_{a, b}$, that it is indeed the case. Let $R_{a, b}^{\prime}$ the deformation ring including this condition at $p$. It is precisely the deformation ring studied in [GT05, § 4.1]. The natural surjection $R_{a, b} \rightarrow R_{a, b}^{\prime}$ is an isomorphism.

Similarly, $\mathbf{V} / P \mathbf{V}=\mathbf{V}_{a, b}$ is Pontryagin dual to $H^{3}\left(S_{K^{p} \times I_{1}}, V_{a, b}(F / \mathcal{O})\right)_{\mathfrak{m}}$.

Let $\mathbf{V}_{a, b}^{\prime}$ be the dual of $H^{3}\left(S_{K^{p} \times G\left(\mathbb{Z}_{p}\right)}, V_{a, b}(F / \mathcal{O})\right)_{\mathfrak{m}^{\prime}}$ and $\mathbf{T}_{a, b}^{\prime}$ be the $\mathcal{O}$-algebra generated by the action of $\mathcal{H}^{N p}$ on $\mathbf{V}_{a, b}^{\prime}$. Recall that we write $\mathbf{T}_{a, b}^{\prime}=\mathbf{T} / P \mathbf{T}$. There is a natural surjective $\mathcal{O}$-algebras homomorphism $\mathbf{T}_{a, b} \rightarrow \mathbf{T}_{a, b}^{\prime}$ inserted in the commutative square

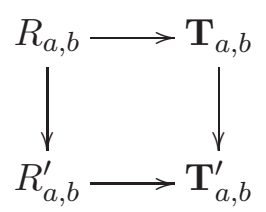

By [GT05, Theorem 4.3.9], the canonical local $\mathcal{O}$-algebras homomorphism $\phi_{a, b}^{\prime}: R_{a, b}^{\prime} \rightarrow \mathbf{T}_{a, b}^{\prime}$ is an isomorphism and $\mathbf{V}_{a, b}^{\prime}$ is finite free over $\mathbf{T}_{a, b}^{\prime}$. In particular, $\mathbf{T}_{a, b} \rightarrow \mathbf{T}_{a, b}^{\prime}$ is bijective. This identification is compatible with the homomorphism $\Psi: H^{3}\left(S_{K^{p} \times G\left(\mathbb{Z}_{p}\right)}, V_{a, b}(F / \mathcal{O})\right)_{\mathfrak{m}^{\prime}} \rightarrow H^{3}\left(S_{K^{p} \times I_{1}}\right.$, $\left.V_{a, b}(F / \mathcal{O})\right)_{\mathfrak{m}}$. By Proposition 3.2, $\Psi$ is an isomorphism. Hence, we have compatible identifications $\mathbf{T}_{a, b}=\mathbf{T}_{a, b}^{\prime}$ and $\mathbf{V}_{a, b}=\mathbf{V}_{a, b}^{\prime}$.

Hence, $\phi_{a, b}=\phi \otimes I d_{\Lambda / P}$ is an isomorphism and $\mathbf{V} / P \mathbf{V}$ is free over $\mathbf{T} / P \mathbf{T}$. This implies that $\mathbf{V}$ is free over $\mathbf{T}$. Indeed by Nakayama's lemma, we have a surjective $\mathbf{T}$ linear homomorphism $\psi: \mathbf{T}^{s} \rightarrow \mathbf{V}$. To show that its kernel is 0 , we reduce modulo $P$, observing that since $\mathbf{V}$ is free over $\Lambda$, the kernel of the quotient map is the quotient of the kernel. Since the kernel of the quotient map is zero, we conclude by Nakayama's lemma that $\psi$ is an isomorphism. 


\section{J. Tilouine}

This implies, in particular, that $\mathbf{T}$ is finite free over $\Lambda$; hence, we can repeat the previous argument to deduce from the fact that $\phi \otimes I d_{\Lambda / P}$ is an isomorphism that $\phi$ itself is injective. So far, we know that $\mathbf{V}$ is free over $\mathbf{T}$ and that $\phi$ is an isomorphism and that the rings are finite flat over $\Lambda$. Since the ideal $P$ is generated by a regular sequence, we infer from the fact that $R / P R$ is complete intersection over $\mathcal{O}$ that the same holds for $R$ over $\Lambda$. Finally, the exact control for any dominant weight $\left(a^{\prime}, b^{\prime}, ; c^{\prime}\right)$ follows by the $\Lambda$-freeness of $\mathbf{T}$.

Corollary 3.3. Let $m$ be the multiplicity of $\pi$. For any dominant algebraic weight $\left(a^{\prime}, b^{\prime}\right)$ (with $c^{\prime}=a^{\prime}+b^{\prime}$ omitted in the notation), there is a $\Gamma$ and $\mathbf{T}_{a^{\prime}, b^{\prime}} \otimes \overline{\mathbb{Q}}_{p}$-equivariant isomorphism

$$
\left(\mathbf{V}_{a^{\prime}, b^{\prime}} \otimes \overline{\mathbb{Q}}_{p}\right)^{\vee} \cong\left(\rho_{\mathbf{T}_{a^{\prime}, b^{\prime}}} \otimes \overline{\mathbb{Q}}_{p}\right)^{\oplus m}
$$

where $\left(\mathbf{V}_{a^{\prime}, b^{\prime}} \otimes \overline{\mathbb{Q}}_{p}\right)^{\vee}$ denotes the contragredient $\overline{\mathbb{Q}}_{p}$-representation of $\mathbf{V}_{a^{\prime}, b^{\prime}} \otimes \overline{\mathbb{Q}}_{p}$.

Proof. This follows from [GT05, Theorem 4.3.5] (more precisely from point (2) in its proof). By the previous theorem, $\mathbf{V}$ is $\mathbf{T}$-free; its rank is equal to that of $\mathbf{V}_{a, b}$ over $\mathbf{T}_{a, b}$. By the main theorem of [GT05], it is equal to $4 \mathrm{~m}$. In particular, by [GT05, Theorem 4.3.5] applied in weight $\left(a^{\prime}, b^{\prime}\right)$, all representations $\pi^{\prime}$ occurring in $H^{3}\left(S_{K^{p} \times I_{1}}, V_{a^{\prime}, b^{\prime}}\right)_{\mathfrak{m}}$ are stable at $\infty$ and have the same multiplicity. Moreover, if $F_{\pi^{\prime}}$ denotes the $p$-adic field image of $\theta_{\pi^{\prime}} \otimes I d_{\mathbb{Q}}$, the following Galois-equivariant decompositions hold

$$
\left(\mathbf{V}_{a^{\prime}, b^{\prime}} \otimes \overline{\mathbb{Q}}_{p}\right)^{\vee} \cong \bigoplus_{\pi^{\prime}}\left(\rho_{\pi^{\prime} / \overline{\mathbb{Q}}_{p}} \otimes F_{\pi^{\prime}}^{m}\right) \cong\left(\rho_{\mathbf{T}_{a^{\prime}, b^{\prime}}} \otimes \overline{\mathbb{Q}}_{p}\right)^{\oplus m}
$$

Indeed, see Part 2 of the proof of Theorem 4.3.5 of [GT05]. This concludes the proof.

\section{4. $p$-adic Siegel modular forms}

\subsection{Hida's control theorem}

In this section, we recall some notation and results from [Hid02] in the case of $p$-adic Siegel cusp forms. Let $K=K^{p} \times K_{p}^{\max }$; it is a congruence subgroup of level $N \geqslant 1$. Recall that by our choice of the local components of $K$ (in $\S 1.2$ ), we know that $K \subset G(\widehat{\mathbb{Z}}), K$ contains the unipotent radical $U^{\prime}(\widehat{\mathbb{Z}})$ of the Siegel parabolic and $\nu(K)=\widehat{\mathbb{Z}}^{\times}$.

This implies (by Mumford theory) that there is a smooth quasi-projective geometrically connected $\mathbb{Z}\left[\frac{1}{N}\right]$-scheme $X$ whose generic fiber over $\mathbb{C}$ is $S_{K}$. Indeed $X=X(N) / K$ is a coarse moduli scheme for isomorphism classes of $(A, \lambda, \bar{\eta})_{/ S}$ where $A / S$ is an abelian scheme, $\lambda$ a principal polarization and $\bar{\eta}$ is a section of the sheaf of $K$-orbits of full level $N$-structures, that is, a section of $\underline{\text { Isom }}\left((\mathbb{Z} / N \mathbb{Z})^{4}, A[N]\right) / K$ where Isom denotes the $G$-torsor of symplectic similitudes (for the standard symplectic pairing on the left-hand side and the $\lambda$-Weil pairing on the right-hand side) and $K$ acts on the right via $K \rightarrow G(\mathbb{Z} / N \mathbb{Z})$ acting on column vectors of $(\mathbb{Z} / N \mathbb{Z})^{4}$. The geometric connectedness of $X$ comes from the surjectivity of $\nu: K \rightarrow \widehat{\mathbb{Z}}^{\times}$.

Let $f: A \rightarrow X(N)$ be the universal principally polarized abelian scheme with full level $N$-level structure $\eta$. We put $\underline{\omega}=e^{*} \Omega_{A / X(N)}$, where $e$ denotes the unit section.

As usual in the theory of coarse moduli, one first defines objects over $X(N)$ and then checks that they descend to $X$. In this vein, let us sketch the construction of automorphic vector bundles, of arithmetic toroidal compactification, and canonical extension of automorphic vector bundles to it.

For any dominant weight $\kappa=(k, \ell ; m)$ of the standard Levi $M^{\prime}$ of the Siegel parabolic, with $k \geqslant \ell, m \equiv k+\ell(\bmod 2)$, we consider the representation $\rho_{\kappa}$ of highest weight $\kappa$; it acts on $W_{\kappa}=\operatorname{Sym}^{k-\ell} \otimes \operatorname{det}^{\ell} \mathrm{St}_{2} \otimes \nu^{(m-k-\ell) / 2}$ of $M^{\prime}$ (with central character $\left.z \mapsto z^{m}\right)$. Here, St 2 denotes the standard two-dimensional representation of GL(2) and, as usual, $\nabla$ denotes the external tensor product: if we are given a representation $V_{1}$ (respectively $V_{2}$ ) of a group $G_{1}$ (respectively $G_{2}$ ), $V_{1} \otimes V_{2}$ 


\section{NEARLY ORDINARY RANK FOUR GALOIS REPRESENTATIONS}

denotes the representation of $G_{1} \times G_{2}$ on the tensor product space $V_{1} \otimes V_{2}$. Here, $G_{1}=G_{2}$, $G_{2}=\mathrm{GL}(1)$, and $M^{\prime}$ is identified to $\mathrm{GL}(2) \times \mathrm{GL}(1)$ by

$$
(A, \nu) \mapsto \operatorname{diag}\left(A, s^{t} A^{-1} s \nu\right) .
$$

The twist by $s$ occurs because our choice of the symplectic matrix $J$ defining $G$ involves the matrix $s$ instead of $1_{2}$.

Then, one can define an automorphic vector bundle $\underline{\omega}^{\kappa}$ over $X$ whose sections are functions on $\operatorname{Isom}_{X}\left(\mathcal{O}_{X}^{2}, \underline{\omega}\right)$ such that for any $\phi \in \operatorname{Isom}_{X}\left(\mathcal{O}_{X}^{2}, \underline{\omega}\right), f\left(A, \lambda, \eta, \phi \circ s U^{-1} s\right)=\rho_{\kappa}(U) f(A, \lambda, \eta, \phi)$ where $\rho_{\kappa}$ denotes the representation of $M^{\prime}$ on $W_{\kappa}$.

Let $S_{2}(L)$ be the $L$-vector space of $2 \times 2$ symmetric matrices. Let $\mathcal{P}_{2}$ be the cone of definite positive matrices in $S_{2}(\mathbb{R})$ and $\partial_{2}$ the cone of semi-definite positive matrices of rank less than 2 whose kernel is $\mathbb{Q}$-rational. Consider $S_{2}^{*}=\mathcal{P}_{2} \cup \partial_{2}$. Any proper $\mathbb{Q}$-quotient $X_{\xi}$ of $\mathbb{Q}^{2}$ is called a rational boundary component of $S_{2}^{*}$. We denote by $R B C$ the set of indices $\xi$ of the rational boundary components. We can partition $\partial_{2}$ as $\bigsqcup_{\xi \in R B C} S\left(X_{\xi}\right)^{+}$where $S\left(X_{\xi}\right)^{+}$denotes the set of semidefinite symmetric matrices which induce a positive definite quadratic form on $X_{\xi}$.

Let $\Sigma=\left\{\Sigma_{\xi}\right\}_{\xi \in R B C}$ be an $\mathrm{Sp}_{4}(\mathbb{Z})$-admissible family of rational polyhedral cone decompositions $\Sigma_{\xi}$ of $S\left(X_{\xi}\right)^{+}$(see [Cha85, ch. I, Definition 5.8.2]). To $\Sigma$, one can associate a toroidal compactification $\bar{X}(N)$ over $\mathbb{Z}\left[\frac{1}{N}\right]$ of our adelic Siegel variety $X(N)$ exactly as the authors do in [FC90, Theorem IV.6.7] in the non-adelic case. This adelic compactification is smooth if $\Sigma$ is sufficiently fine; this is assumed in the sequel. Moreover, it carries an action of $G(\mathbb{Z} / N \mathbb{Z})$. Indeed the construction carried out in [FC90, Theorem IV.6.5, p. 128] can be made adelic: the adelic $X(N)$ is obtained from the scheme $A_{2, N}$ in [FC90] by forgetting the reference to a specific primitive $N$ th root of unity in the moduli problem; it therefore carries an action of $G(\mathbb{Z} / N \mathbb{Z})$. Then in the notation of [FC90, p. 128], one defines the adelic version of the scheme $U_{N}$ by normalization of $U_{1}$ in the adelic $X(N)$, and similarly the adelic equivalence relation $R_{N}$; the quotient $U_{N} / R_{N}$ has an action of $G(\mathbb{Z} / N \mathbb{Z})$ instead of $\operatorname{Sp}(4, \mathbb{Z} / N \mathbb{Z}$ ) (by functoriality of the normalization). The compactification $\bar{X}(N)$ carries a degenerating semiabelian scheme $\mathcal{G}$ extending $A$ (see [FC90, Theorem IV.5.7 and IV.6.7]). One still denotes by $\underline{\omega}$ the sheaf $e^{*} \Omega_{\mathcal{G} / \bar{X}(N)}$ where $e$ is the unit section of $\mathcal{G} \rightarrow \overline{X(N)}$.

Let $\bar{X}$ be the quotient of $\bar{X}(N)$ by $K$; it is a projective smooth, geometrically connected scheme over $\mathbb{Z}\left[\frac{1}{N}\right]$. It is endowed with a projection map $b$ to the minimal compactification $X_{\mathbb{Z}\left[\frac{1}{N}\right]}^{*}$. Let $D=$ $\bar{X} \backslash X=b^{-1}\left(\partial X^{*}\right)$; it is a relative Cartier divisor with normal crossings; its irreducible components are smooth.

The rank two vector bundle $\underline{\omega}$ descends to $\bar{X}$ (as a vector bundle) but not to $X^{*}$.

The $\mathrm{GL}_{2}$-torsor $\overline{\mathcal{T}}=\operatorname{Isom}_{\bar{X}}\left(\mathcal{O}_{\bar{X}}^{2}, \underline{\omega}\right)$ allows one to define 'the canonical extensions' of the vector bundles $\underline{\omega}^{\kappa}$ to $\bar{X}$ : for any $\mathrm{GL}_{2}$-dominant weight $\kappa=(k, \ell), k \geqslant \ell$, and $W_{\kappa}$ an integral structure of the corresponding irreducible representation of $\mathrm{GL}_{2}$, one puts $\underline{\omega}^{\kappa}=\overline{\mathcal{T}} \stackrel{\mathrm{GL}_{2}}{\times} W_{\kappa}$, where as usual the contraction product is the quotient of the product by the equivalence relation $(\phi \circ g, w) \sim(\phi, g \cdot w)$ for any $\phi \in \overline{\mathcal{T}}, g \in \mathrm{GL}_{2}$ and $w \in W_{\kappa}$. Since there is, in general, an ambiguity for the integral structure of $W_{\kappa} \otimes \mathbb{Q}$, we need to make our choice explicit: following [Hid02, §3], we take the integral induction $W_{\kappa}=\operatorname{Ind}_{B_{\mathrm{GL}_{2}}}^{\mathrm{GL}_{2}} \kappa$. For details, see [FC90, ch. 4 and 6], [MT02, §4] and [Hid02, §3].

Let $\underline{\omega}_{\kappa}=\underline{\omega}^{\kappa}(-D)$ be the sub-vector bundle of $\underline{\omega}^{\kappa}$ on $\bar{X}$ whose sections vanish along $D$. Recall that $H^{0}\left(X \otimes \mathbb{C}, \underline{\omega}^{\kappa}\right)$ (respectively $\left.H^{0}\left(X \otimes \mathbb{C}, \underline{\omega}_{\kappa}\right)\right)$ identifies canonically to the space of Siegel modular forms of level $K$ and weight $\kappa$ (respectively its subspace of cusp forms); (see [Hid02, Theorem 3.1]).

For any integer $m \geqslant 1$, let $X_{m}$ be the pull-back of $X$ to $\mathbb{Z} / p^{m} \mathbb{Z}$. Let $S_{m}$ be the ordinary locus and for each $n \geqslant 1$, consider $T_{m, n}=\operatorname{Isom}_{S_{m}}\left(\mu_{p^{n}}^{2}, A\left[p^{n}\right]^{0}\right)=\operatorname{Isom}_{S_{m}}\left(A\left[p^{n}\right]^{\text {ét }},\left(\mathbb{Z} / p^{n} \mathbb{Z}\right)^{2}\right)$; for any $n \geqslant 1$, $T_{m, n}$ is a connected Galois cover of $S_{m}$ of Galois group $\mathrm{GL}_{2}\left(\mathbb{Z} / p^{n} \mathbb{Z}\right.$ ) (see [FC90, Proposition 7.2]). 


\section{J. Tilouine}

Let $M_{1}^{\prime}$ be the subgroup of the Siegel Levi $M^{\prime}$ corresponding to GL(2) in the isomorphism (4.1.1). Let $\mathbb{M}_{1}^{\prime}=\mathrm{GL}_{2}\left(\mathbb{Z}_{p}\right)$ be its group of $\mathbb{Z}_{p}$-points. It is the Galois group of $T_{m, \infty}$ over $S_{m}$ and $T_{m, n}$ is an $M_{1}^{\prime}\left(\mathbb{Z} / p^{n} \mathbb{Z}\right)$-torsor over $S_{m}$.

Remark. We could construct a torsor over a non-constant group scheme fpqc-locally isomorphic to $M^{\prime}\left(\mathbb{Z} / p^{n} \mathbb{Z}\right)$ by replacing $T_{m, n}$ by $T_{m, n}^{\prime}=T_{m, n} \times \mu_{p^{n}}^{\times}$where $\mu_{p^{n}}^{\times}$is the finite flat scheme of primitive $p^{n}$ th roots of unity. The finite morphism $T_{m, n}^{\prime} \rightarrow S_{m}$ is still connected, the Galois of group $M^{\prime}\left(\mathbb{Z} / p^{n} \mathbb{Z}\right)=\mathrm{GL}_{2}\left(\mathbb{Z} / p^{n} \mathbb{Z}\right) \times\left(\mathbb{Z} / p^{n} \mathbb{Z}\right)^{\times}$but is no longer étale over $S_{m}$. Correspondingly, the rigid space associated to $T_{\infty, \infty}^{\prime}$ is étale over $S_{\infty}$ but its group of connected components is isomorphic to $\mathbb{Z}_{p}^{\times}$. For simplicity of notations, we will not do that and stick to the $M_{1}^{\prime}$-torsors $T_{m, n}$.

Let $V_{m, n}=H^{0}\left(T_{m, n}, \mathcal{O}_{T_{m, n}}\right), V_{m, \infty}=\bigcup_{n \geqslant 1} V_{m, n}$. One can define the $\Sigma$-'toroidal compactification' $\bar{S}_{m}$ of $S_{m}$ as the locus of $\bar{X}_{m}$ over which $\mathcal{G}[p]^{0}$ is of multiplicative type; similarly, define $\bar{T}_{m, n}$ as the quotient by $K$ of

$$
\operatorname{Isom}_{\bar{X}(N) \otimes \mathbb{Z} / p^{m} \mathbb{Z}}\left(\mu_{p^{n}}^{2}, \mathcal{G}\left[p^{n}\right]^{0}\right)
$$

(its action being trivial on $\mu_{p^{n}}^{2}$ and $\mathcal{G}\left[p^{n}\right]^{0}$ ). We still denote by $D$ the pull-back of the divisor at $\infty$. We can now define $V_{!, m, n}=H^{0}\left(T_{m, n}, \mathcal{O}_{T_{m, n}}(-D)\right)$ and $V_{!, m, \infty}=\bigcup_{m} V_{!, m, n}$. We also consider the

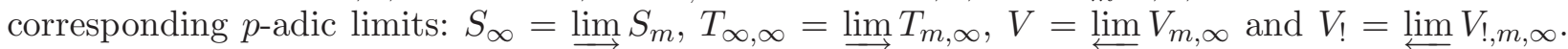

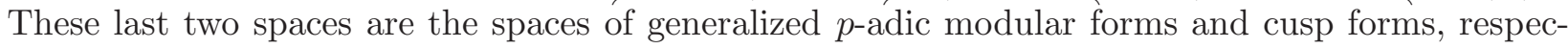
tively.

Recall that the bilinear form $\operatorname{Tr}: S_{2}(\mathbb{R}) \times S_{2}(\mathbb{R}) \rightarrow \mathbb{R}$ on the space of symmetric $2 \times 2$-matrices identifies the dual of $S_{2}(\mathbb{Z})$ to the module $\mathcal{S}$ of matrices $\left(\begin{array}{cc}a & \frac{1}{2} b \\ \frac{1}{2} b & c\end{array}\right), a, b, c \in \mathbb{Z}$. Let $\mathcal{S}^{+}=\mathcal{S} \cap S_{2}(\mathbb{R})^{+}$ be the cone of semi-definite matrices in $\mathcal{S}$. Let $\eta \in R B C$ such that $X_{\eta}=0$. Let $\Sigma_{\eta}$ be the $\mathrm{GL}_{2}(\mathbb{Z})$ admissible rational polyhedral cone decomposition of $\mathcal{P}_{2}$ induced by $\Sigma$. Let $R=\mathbb{Z}_{p}\left[\left[q^{T} ; T \in \mathcal{S}^{+}\right]\right]=$ $\bigcap_{\sigma \in \Sigma_{\eta}} \mathbb{Z}_{p}\left[\left[q^{T} ; T \in \sigma^{\vee} \cap \mathcal{S}^{+}\right]\right]$and let $\mathcal{C}_{\mathbb{M}_{1}^{\prime}}$ be the ring of $\mathbb{Z}_{p^{-}}$valued continuous functions on $\mathbb{M}_{1}^{\prime} / \mathbb{N}$ $\left(\mathbb{N}^{\prime}\right.$ is the group of $\mathbb{Z}_{p}$-points of upper triangular unipotent matrices in $M_{1}^{\prime}$ ).

The $q$-extension map is a ring homomorphism

$$
\mathrm{FE}: V^{\mathbb{N}^{\prime}} \rightarrow \mathcal{C}_{\mathbb{M}_{1}^{\prime}} \widehat{\otimes} R
$$

given by $f \mapsto \pi_{\eta}^{*} f$ where $\pi_{\eta}: \mathcal{S}_{\eta} \rightarrow \bar{X}^{\wedge}$ is a canonical étale formal scheme morphism from the local chart $\mathcal{S}_{\eta}$ to the formal completion of $\bar{X}$ along the divisor $D$. The morphism $\pi_{\eta}$ is characterized as follows (for details, see [FC90, pp. 103, 104 and 106]). The pull-back of $\mathcal{G}$ by $\pi_{\eta}$ is the canonical Mumford family

$$
f_{\eta}: \mathcal{G}_{\eta} \rightarrow \mathcal{S}_{\eta}
$$

deduced by Mumford's construction (see [FC90, p. 54]) from the canonical degenerescence data in $\mathrm{DD}_{\text {ample }}$ on the global torus $\tilde{G}_{\eta}=\mathbf{G}_{m}^{2}$ over $\mathcal{S}_{\eta}$, together with the standard level $N$ structure $\mu_{N}^{2} \times(\mathbb{Z} / N \mathbb{Z})^{2} \rightarrow \tilde{G}_{\eta}[N]$; in particular, there is a canonical rigidification $\psi_{\text {can }}: \mu_{p^{\infty}}^{2} \cong \mathcal{G}_{\eta}\left[p^{\infty}\right]^{0}$ induced from the tautological rigidification of $\tilde{G}_{\eta}$.

For details on the map FE, see [Til06, §3]. In particular, FE sends forms of diagonal weight $\kappa=(k, k)$ into the $R$-line $\operatorname{det}^{k} \otimes R$, so that if $\left(f_{i}\right)$ is a sequence of forms of $p$-adically converging diagonal weights, the images $\mathrm{FE}\left(f_{i}\right)$ can be considered as belonging to $R$. This will be used in the sequel.

The ring homomorphism $\mathrm{FE}$ is injective with flat cokernel. Indeed, for any $m \geqslant 1, V / p^{m} V \subset$ $V_{m, \infty} \hookrightarrow R / p^{m} R$ by the irreducibility of $T_{m, \infty}$.

However, the surjectivity of $V / p^{m} V \hookrightarrow V_{m, \infty}$ is more subtle than in the genus one case. It is not known in general. It is only known for its restriction to the ideal $V_{\text {! }}$ of cusp forms. Actually, by [Hid02, Theorem 3.1], we have $V_{!} / p^{m} V_{!}=V_{!, m, \infty}$ and $\mathrm{FE} \mid V_{!}$has flat cokernel. 


\section{NEARLY ORDINARY RANK FOUR GALOIS REPRESENTATIONS}

Let $H \in H^{0}\left(X_{1}, \operatorname{det}^{p-1} \underline{\omega}\right)$ be the Hasse invariant on $X_{1}$. We fix an integer $t \geqslant 1$ sufficiently large such that $H^{t}$ lifts to $X$ over $\mathbb{Z}_{p}$. This can be achieved because det $\underline{\omega}$ is ample. We denote such a lifting by $E$. Recall that $\mathrm{FE}(E) \equiv 1(\bmod p)$.

Indeed, one has $\mathrm{FE}(H)=1$. This follows from a direct calculation for the Mumford family $f_{\eta}: \mathcal{G}_{\eta} \rightarrow \mathcal{S}_{\eta}$. By one of the characterizations of the Hasse invariant, it is enough to show that the Frobenius endomorphism on the dual of $\underline{\omega}_{\mathcal{G}_{\eta} / \mathcal{S}_{\eta}} \otimes \mathbb{F}_{p}$ is the identity (tensored by the Frobenius of $\mathcal{S}_{\eta} \otimes \mathbb{F}_{p}$ ). For that purpose, by Mumford's construction it is enough to prove this for the constant torus $\mathbf{G}_{m}^{2}$ over $\mathcal{S}_{\eta}$. Then, $\underline{\omega}_{\mathbf{G}_{m}^{2} / \mathcal{S}_{\eta}}^{\vee} \otimes \mathbb{F}_{p}$ identifies to the module of $\mathbf{G}_{m}^{2}$-translation invariant derivations on $\left(\mathbf{G}_{m}^{2} \times \mathcal{S}_{\eta}\right) \otimes \mathbb{F}_{p}$; which admits a canonical basis $D_{i}=q_{i}\left(d / d q_{i}\right)(=1,2)$. The Frobenius endomorphism acts on this basis by $D_{i} \mapsto D_{i}^{p}$ (composition $p$ times). $D_{i}^{p}$ and $D_{i}$ agree on the variables $q_{1}$ and $q_{2}$, hence $D_{i}^{p}=D_{i}$, so that the matrix of Frobenius is the identity.

Let $B_{1}^{\prime}=T_{1} N^{\prime}$ be the Levi decomposition of the standard Borel of $M_{1}^{\prime}$; here, $T_{1}$ denotes the standard maximal torus of the semisimple part of $G$; it is also a maximal torus of $M_{1}^{\prime}$.

We write $\mathbb{B}_{1}^{\prime}=\mathbb{T}_{1} \mathbb{N}^{\prime}$ for the decomposition of the corresponding groups of $\mathbb{Z}_{p}$-points. Then $V_{!}^{\mathbb{N}^{\prime}}=H^{0}\left(T_{\infty, \infty} / \mathbb{N}^{\prime}, \mathcal{O}_{T_{\infty, \infty}}^{\mathbb{N}^{\prime}}(-D)\right)$; it is the space of $p$-adic Siegel cusp forms of auxiliary level $K^{p}$ with infinite 'strict Iwahori' $p$-level structure (one could also say $\Gamma_{I}\left(p^{\infty}\right)$-level structure).

Igusa's irreducibility theorem implies that $T_{\infty, \infty} / \mathbb{N}^{\prime} \rightarrow T_{\infty, \infty} / \mathbb{B}_{1}^{\prime}$ is an étale Galois cover with Galois group $\mathbb{T}_{1}$.

Definition 4.1. Let $\Lambda_{1}=\mathbb{Z}_{p}\left[\left[\mathbb{T}_{1}^{+}\right]\right]$be the Iwasawa algebra associated to the pro- $p$-Sylow $\mathbb{T}_{1}^{+}$of $\mathbb{T}_{1}$. $\mathbb{T}$ acts continuously on $V_{\text {! }}$ and $V_{!}^{\mathbb{N}^{\prime}}$; by linearity and continuity, the algebra $\Lambda_{1}$ acts continuously on $V_{\text {! }}$ and $V_{!}^{\mathbb{N}^{\prime}}$. Write $i: \Lambda_{1} \hookrightarrow \Lambda$ for the injection of rings induced by

$$
i:\left[\operatorname{diag}\left(t_{1}, t_{2}, t_{2}^{-1}, t_{1}^{-1}\right)\right] \mapsto t_{1}^{3} t_{2}^{3}\left[\operatorname{diag}\left(t_{1}, t_{2}, t_{2}^{-1}, t_{1}^{-1}\right)\right] .
$$

In the sequel, we still denote by $P_{a^{\prime}, b^{\prime}}$ the prime ideal of $\Lambda_{1}$ inverse image by $i$ in $\Lambda_{1}$ of the prime ideal $P_{a^{\prime}, b^{\prime}}$ of $\Lambda$.

\section{Comments.}

(1) Note that the inclusion $i$ induces an isomorphism:

$$
\Lambda_{1} / P_{a^{\prime}, b^{\prime}} \cong \Lambda / P_{a^{\prime}, b^{\prime}}
$$

(2) The motivation for the twist occurring in $i$ is the following: for any (neat) compact open subgroup $L$ of $G_{f}$, for $a^{\prime} \geqslant b^{\prime} \geqslant 0$ and $k^{\prime}=a^{\prime}+3, \ell^{\prime}=b^{\prime}+3$, there is a canonical Heckeequivariant embedding

$$
H^{0}\left(S_{L}, \underline{\omega}_{k^{\prime}, \ell^{\prime}}\right) \hookrightarrow H^{3}\left(S_{L}, V_{a^{\prime}, b^{\prime}}(\mathbb{C})\right)
$$

(see [Hid02, §3.8] where it is explained how to make it canonical, and where it is called the Eichler-Shimura map).

By $[$ Hid02, $\S 3.6]$, the Hecke operators $U_{p, 1}=\left[\mathbb{N}^{\prime} \operatorname{diag}(1,1, p, p) \mathbb{N}^{\prime}\right]$ and $U_{p, 2}=p^{-4}$ $\left[\mathbb{N}^{\prime} \operatorname{diag}\left(1, p, p, p^{2}\right) \mathbb{N}^{\prime}\right]$ act on $V_{!}^{\mathbb{N}^{\prime}}$. Let $e=\lim \left(U_{p, 1} U_{p, 2}\right)^{n !}$ be the corresponding idempotent of $\operatorname{End}_{\mathbb{Z}_{p}} V_{!}^{\mathbb{N}^{\prime}}$. Hida's control theorem [Hid02, Theorem 1.1] is a descent theorem from $T_{\infty, \infty} / \mathbb{N}^{\prime}$ to $S_{\infty}$ for ordinary cusp forms of strict Iwahori $p$-level. Let $\widetilde{\mathcal{S}}^{*}\left(K^{p}\right)=\operatorname{Hom}\left(e V_{!}^{\mathbb{N}^{\prime}} \otimes_{\Lambda_{1}, i} \Lambda, \mathbb{Z}_{p}\right)$ be the $\mathbb{Z}_{p}$-dual of $e V_{!}^{\mathbb{N}^{\prime}} \otimes_{\Lambda_{1}, i} \Lambda$.

Theorem 5. The $\Lambda$-module $\widetilde{\mathcal{S}}^{*}\left(K^{p}\right)$ is finite and free; moreover, for any pair $\left(a^{\prime}, b^{\prime}\right)$ of integers such that $a^{\prime} \geqslant b^{\prime} \geqslant-3$, and $\kappa=(k, \ell)$ with $k=a^{\prime}+3$ and $\ell=b^{\prime}+3$, one has a canonical Hecke-linear isomorphism

and

$$
\widetilde{\mathcal{S}}^{*}\left(K^{p}\right) / P_{a^{\prime}, b^{\prime}} \widetilde{\mathcal{S}}^{*}\left(K^{p}\right) \cong \operatorname{Hom}\left(e H^{0}\left(S_{\infty}, \underline{\omega}_{\kappa}\right), \mathbb{Z}_{p}\right)
$$

$$
e V_{!}^{\mathbb{N}^{\prime}}\left[P_{a^{\prime}, b^{\prime}}\right]=\left(e V_{!}^{\mathbb{N}^{\prime}} \otimes_{\Lambda_{1}, i} \Lambda\right)\left[P_{a^{\prime}, b^{\prime}}\right]=e H^{0}\left(S_{\infty}, \underline{\omega}_{\kappa}\right)
$$




\section{J. Tilouine}

Proof. Let us put $\mathfrak{V}=\operatorname{Hom}\left(e V_{!}^{\mathbb{N}^{\prime}}, \mathbb{Z}_{p}\right)=\operatorname{Hom}\left(\lim _{\longleftarrow} V_{!, m}^{\mathbb{N}^{\prime}}, \mathbb{Z}_{p}\right)$.

In [Hid02, Theorem 1.1], is stated for the module $\widetilde{\mathfrak{V}}^{\prime}=\operatorname{Hom}\left(\stackrel{\lim }{\longrightarrow} V_{!, m}^{\mathbb{N}^{\prime}}, \mathbb{Q}_{p} / \mathbb{Z}_{p}\right)$. However, recall that $\left[\right.$ Hid02, $\S 3.7$, Proposition 3.6] shows that the canonical homomorphisms $V_{!}^{\mathbb{N}^{\prime}} / p^{m} V_{!}^{\mathbb{N}^{\prime}} \rightarrow V_{!, m}^{\mathbb{N}^{\prime}}$ are isomorphisms. They induce a canonical isomorphism

$$
\mathfrak{V}^{\prime}=\operatorname{Hom}\left(\underline{\lim } V_{!, m}, \mathbb{Q}_{p} / \mathbb{Z}_{p}\right) \cong \mathfrak{V}=\operatorname{Hom}\left(\lim _{\longleftarrow} V_{!, m}, \mathbb{Z}_{p}\right)
$$

similarly, the same proposition [Hid02, Proposition 3.6] provides canonical isomorphisms $H^{0}\left(S_{\infty}, \underline{\omega}_{\kappa}\right) / p^{m} H^{0}\left(S_{\infty}, \underline{\omega}_{\kappa}\right) \cong H^{0}\left(S_{m}, \underline{\omega}_{\kappa}\right)$ that induce a canonical isomorphism

$$
\mathfrak{V} / P_{a^{\prime}, b^{\prime}} \mathfrak{V} \cong \operatorname{Hom}\left(e H^{0}\left(S_{\infty}, \underline{\omega}_{\kappa}\right), \mathbb{Z}_{p}\right)
$$

for $\kappa=\left(a^{\prime}+3, b^{\prime}+3\right) \in X^{*}\left(T_{1}\right)$. In fact, one finds in [Hid02, $\S 3.7$, p. 39] (more precisely, in the key point of its proof) that one has $e V_{!}^{\mathbb{N}^{\prime}}\left[P_{a^{\prime}, b^{\prime}}\right]=e H^{0}\left(S_{\infty}, \underline{\omega}_{\kappa}\right)$. The theorem follows.

Actually, [Hid02, Theorem 1.1] also contains a 'classicity statement', but only for very regular weights. Since we will need an analog of this statement for singular weights $(k=\ell)$, we prove it in the next section.

\subsection{A conjecture of Hida}

In this section, we prove a stronger version of [Hid02, Conjecture 7.2] but only after localization at our non-Eisenstein maximal ideal $\mathfrak{m}^{\prime}$ of the Hecke algebra outside $N p$. Let $e_{0}$ be the idempotent

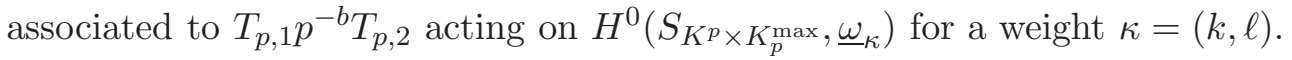

Conjecture. For any weight $\kappa$ such that $k \geqslant \ell>3$, the canonical inclusion followed by the idempotent $e$ induces an isomorphism

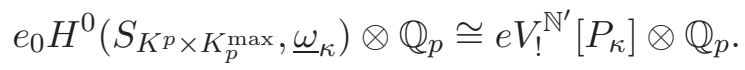

Theorem 6. The conjecture is true for the localizations at $\mathfrak{m}^{\prime}$ of the $\mathbb{Q}_{p}$-vector spaces above.

Remark. In [Hid02], the conjecture is stated for more general groups but involves a stronger condition of regularity for $\kappa$; namely, in the case of $\mathrm{GSp}_{4}$, it would require $k>\ell+2$ and $\ell>0$.

Proof. Let $e_{0}$ be the idempotent of $\operatorname{End}\left(H^{3}\left(\Gamma, V_{a^{\prime}, b^{\prime}}\left(\mathbb{Q}_{p}\right)\right)\right)$ associated to $T_{p, 1} p^{-b^{\prime}} T_{p, 2}$. The first step, as in the proof of Theorem 1.1(4) in [Hid02], is to show that the sequence $\operatorname{dim}_{\mathbb{Q}_{p}} e_{0} H^{0}\left(X \otimes \mathbb{Q}_{p}, \underline{\omega}_{k^{\prime}, \ell^{\prime}}\right)_{\mathfrak{m}}$ is constant when $\left(a^{\prime}, b^{\prime}\right)$ varies in the set of pairs such that $a^{\prime} \geqslant b^{\prime}>0$ and $a^{\prime} \equiv a(\bmod p-1)$, $b^{\prime} \equiv b(\bmod p-1)$. Recall that a similar statement for Betti cohomology is proven in [TU99, Proposition 3.2]. Indeed, there are two cases: (1) $a>b$ or (2) $a=b$.

In case (1), let $I$ be the $p$-Iwahori subgroup associated to the Siegel parabolic $Q, \Gamma_{Q}(p)=\Gamma \cap I$ and $e_{I}$ be the idempotent associated to the $I$-level Hecke operator $U_{p, 1} \cdot U_{p, 2}$. We use [TU99, Proposition 3.2$]$ for the Iwahori case: For any $a^{\prime}>b^{\prime}>0$ with $a^{\prime} \equiv a(\bmod p-1)$ and $b^{\prime} \equiv b$ $(\bmod p-1)$, the restriction followed by $e_{I}$ induces an isomorphism

$$
e_{0} H^{3}\left(\Gamma, V_{a, b}\left(\mathbb{Q}_{p}\right)\right)_{\mathfrak{m}^{\prime}} \equiv e_{I} H^{3}\left(\Gamma_{Q}(p), V_{a, b}\left(\mathbb{Q}_{p}\right)\right)_{\mathfrak{m}^{\prime}} .
$$

We then apply the exact control theorem, Theorem 2, to the free $\Lambda$-module $e \mathbf{V}$ (defined by localization at the maximal ideal $\mathfrak{m}^{\prime}$ ).

This allows us to conclude that the dimension of $e_{Q} H^{3}\left(\Gamma_{Q}(p), V_{a^{\prime}, b^{\prime}}\left(\mathbb{Q}_{p}\right)\right)_{\mathfrak{m}^{\prime}}$ is independent of the pair $\left(a^{\prime}, b^{\prime}\right)$ varying as above; this yields the series of equalities

$$
\begin{aligned}
\operatorname{dim}_{\mathbb{Q}_{p}} e_{0} H^{3}\left(\Gamma, V_{a, b}\left(\mathbb{Q}_{p}\right)\right)_{\mathfrak{m}^{\prime}} & =\operatorname{dim}_{\mathbb{Q}_{p}} e_{I} H^{3}\left(\Gamma_{I}(p), V_{a, b}\left(\mathbb{Q}_{p}\right)\right)_{\mathfrak{m}^{\prime}} \\
& =\operatorname{dim}_{\mathbb{Q}_{p}} e_{I} H^{3}\left(\Gamma_{I}(p), V_{a^{\prime}, b^{\prime}}\left(\mathbb{Q}_{p}\right)\right)_{\mathfrak{m}^{\prime}}=\operatorname{dim}_{\mathbb{Q}_{p}} e_{0} H^{3}\left(\Gamma, V_{a^{\prime}, b^{\prime}}\left(\mathbb{Q}_{p}\right)\right)_{\mathfrak{m}^{\prime}} .
\end{aligned}
$$

In case (2), let $\Pi_{Q}$ be the $p$-parahoric subgroup associated to the Siegel parabolic $Q, \Gamma_{Q}(p)=$ $\Gamma \cap \Pi_{Q}$ and $e_{Q}$ be the idempotent associated to the $\Gamma_{Q}(p)$-level Hecke operator $U_{p, 1}^{Q} \cdot U_{p, 2}^{Q}$. 


\section{NEARLY ORDINARY RANK FOUR GALOIS REPRESENTATIONS}

Proposition 3.2 of [TU99] now reads: for any $a^{\prime}>0, a^{\prime} \equiv a(\bmod p-1)$, the restriction followed by $e_{Q}$ provides an isomorphism

$$
e_{0} H^{3}\left(\Gamma, V_{a, a}\left(\mathbb{Q}_{p}\right)\right)_{\mathfrak{m}^{\prime}} \equiv e_{Q} H^{3}\left(\Gamma_{Q}(p), V_{a^{\prime}, a^{\prime}}\left(\mathbb{Q}_{p}\right)\right)_{\mathfrak{m}^{\prime}} .
$$

Now, after localization at the maximal ideal $\mathfrak{m}^{\prime}$ of the Hecke algebra outside $N p$, we have a similar Iwasawa module $e \mathbf{V}_{Q, \mathfrak{m}^{\prime}}$. This module is defined in [TU99]. By a proof identical to that of [MT02, $\S 9]$, one shows that (1) it is finite free over the Iwasawa algebra $\Lambda_{Q}$ defined as the completed group algebra of the pro- $p$-Sylow of $C_{Q}\left(\mathbb{Z}_{p}\right)$ where $C_{Q}$ is the cocenter of the Levi of $Q$ (it is a torus of rank two only); (2) it satisfies an exact control theorem analog to Theorem 2, but only for diagonal weights $\left(a^{\prime}, a^{\prime}\right), a^{\prime}>0$.

Therefore,

$$
\begin{aligned}
\operatorname{dim}_{\mathbb{Q}_{p}} e_{0} H^{3}\left(\Gamma, V_{a, a}\left(\mathbb{Q}_{p}\right)\right)_{\mathfrak{m}^{\prime}} & =\operatorname{dim}_{\mathbb{Q}_{p}} e_{Q} H^{3}\left(\Gamma_{Q}(p), V_{a, a}\left(\mathbb{Q}_{p}\right)\right)_{\mathfrak{m}^{\prime}} \\
& =\operatorname{dim}_{\mathbb{Q}_{p}} e_{Q} H^{3}\left(\Gamma, V_{a^{\prime}, a^{\prime}}\left(\mathbb{Q}_{p}\right)\right)_{\mathfrak{m}^{\prime}}=\operatorname{dim}_{\mathbb{Q}_{p}} e_{0} H^{3}\left(\Gamma, V_{a^{\prime}, a^{\prime}}\left(\mathbb{Q}_{p}\right)\right)_{\mathfrak{m}^{\prime}}
\end{aligned}
$$

for any $\left(a^{\prime}, a^{\prime}\right)$ in the set of pairs such that $a^{\prime}>0$ and $a^{\prime} \equiv a(\bmod p-1)$.

Moreover, after localization at $\mathfrak{m}^{\prime}$, we know that all of the automorphic representations occurring in $e_{0} H^{3}\left(\Gamma, V_{a^{\prime}, b^{\prime}}\left(\mathbb{Q}_{p}\right)\right)_{\mathfrak{m}^{\prime}}$ are cuspidal and stable at $\infty$. Therefore, the Eichler-Shimura isomorphism theorem (see, for instance, [FC90, Theorem VI.5.5] or rather the first case of [FC90, VI.6]) implies that

$$
4 m_{\pi} \operatorname{dim}_{\mathbb{Q}_{p}} e_{0} H^{0}\left(X \otimes \mathbb{Q}_{p}, \underline{\omega}_{k^{\prime}, \ell^{\prime}}\right)_{\mathfrak{m}^{\prime}}=\operatorname{dim}_{\mathbb{Q}_{p}} e_{0} H^{3}\left(\Gamma, V_{a^{\prime}, b^{\prime}}\left(\mathbb{Q}_{p}\right)\right)_{\mathfrak{m}^{\prime}}
$$

is constant.

In the second step, we show that for $\kappa=(k, \ell), k \geqslant \ell>3$, the map

$$
e_{0} H^{0}\left(X_{\mathbb{Z}_{p}}, \underline{\omega}_{\kappa}\right) \rightarrow e H^{0}\left(S_{\infty}, \underline{\omega}_{\kappa}\right)
$$

given by $e$ is an isomorphism after tensoring by $\mathbb{Q}_{p}$.

First, by the Hida theorem [Hid02, Theorem 1.1(3)], the right-hand side is of finite rank over $\mathbb{Z}_{p}$, therefore the same holds for the $\mathbb{Z}_{p^{-}}$-submodule $e H^{0}\left(S, \underline{\omega}_{\kappa}\right)$; this shows that the $p$-adic completion is unnecessary: $e H^{0}\left(S, \underline{\omega}_{\kappa}\right)=e H^{0}\left(S_{\infty}, \underline{\omega}_{\kappa}\right)$. In particular, for any $f \in e H^{0}\left(S_{\infty}, \underline{\omega}_{\kappa}\right)$, there exists $m \geqslant 0$ such that $f E^{p^{m}} \in H^{0}\left(X_{\mathbb{Z}_{p}}, \underline{\omega}_{\kappa+t p^{m}(p-1)(1,1)}\right)$. The rank of this $\mathbb{Z}_{p^{-}}$-module in independent of $m$ by the proof of Conjecture 1 .

On the other hand, $h \mapsto e\left(h E^{p^{m}}\right)$ is injective with flat cokernel from $e H^{0}\left(X_{\mathbb{Z}_{p}}, \underline{\omega}_{\kappa}\right)$ to $e H^{0}\left(X_{\mathbb{Z}_{p}}, \underline{\omega}_{\kappa+t p^{m}(p-1)(1,1)}\right)$ Indeed, it is enough to check the injectivity after reduction modulo $p$. By the fact that $E$ reduces to the Hasse invariant $H$ whose $q$-expansion is one, we conclude that $\mathrm{FE}\left(e\left(h E^{p^{m}}\right)\right)=\mathrm{FE}\left(e\left(h E^{p^{m}}\right)\right)=\mathrm{FE}(h)$. By the $q$-expansion principle modulo $p$, this implies the injectivity modulo $p$ of $h \mapsto h E^{p^{m}}$.

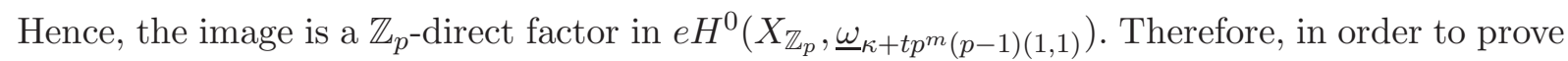
the surjectivity after localizing at $\mathfrak{m}$, it suffices to show the equality of the dimensions of the $\mathbb{Q}_{p}$-vector spaces that they span. They are equal by Step 1 .

Comment. Since $\mathrm{FE}[E] \equiv 1(\bmod p)$, one has $\mathrm{FE}\left[E^{p^{m}}\right] \equiv 1\left(\bmod p^{m+1}\right)$. Moreover in the proof it appears that $\mathrm{FE}\left[e\left(f E^{p^{m}}\right)\right] \equiv \mathrm{FE}\left[e\left(h E^{p^{m}}\right)\right]\left(\bmod p^{m+1}\right)$, with $h$ of level prime to $p$. Hence, we have the following lemma.

Lemma 4.2. For any $f \in e H^{0}\left(S_{\infty}, \underline{\omega}_{\kappa}\right)$, there exists a sequence of prime-to-p level cusp forms $g_{m} \in H^{0}\left(X_{\mathbb{Z}_{p}}, \underline{\omega}_{\kappa+t p^{m}}(p-1)(1,1)\right)$ such that the sequence $\left(\mathrm{FE}\left(g_{m}\right)\right)_{m}$ converges to $\mathrm{FE}(f)$. 


\section{J. Tilouine}

\section{$4.3 \Lambda$-adic Siegel cusp forms}

The abstract Hecke algebra $\mathcal{H}^{N p}$, together with the operators $U_{p, i}(i=1,2)$ defined above do act on $V_{!}^{\mathbb{N}^{\prime}}$. Let $e$ be Hida's idempotent; it is associated to the product of the two operators $U_{p, 1}$ and $U_{p, 2}$. Recall that $\widetilde{\mathcal{S}}^{*}\left(K^{p}\right)=\operatorname{Hom}\left(e V_{!}^{\mathbb{N}^{\prime}} \otimes_{\Lambda_{1}, i} \Lambda, \mathbb{Z}_{p}\right)$ is a module over $\Lambda$ and over $\mathbf{T}$ (in a compatible way). Let $\mathcal{S}^{*}\left(K^{p}\right)$ be the localization at $\mathfrak{m}$ of $\widetilde{\mathcal{S}}^{*}\left(K^{p}\right)$; it is also the $\mathbb{Z}_{p}$-dual of the localization at $\mathfrak{m}$ of $e V_{!}^{\mathbb{N}^{\prime}}$. We define the module $\mathcal{S}\left(K^{p}, \Lambda\right)$ of nearly ordinary $\Lambda$-adic families of Siegel cusp forms as the $\Lambda$-dual

$$
\mathcal{S}\left(K^{p}, \Lambda\right)=\underset{\Lambda}{\operatorname{Hom}}\left(\mathcal{S}^{*}\left(K^{p}\right), \Lambda\right)
$$

of $\mathcal{S}^{*}\left(K^{p}\right)$. By [Hid02, Theorem 1.1(5)], it is a finite free $\Lambda$-module.

Lemma 4.3. There exists a unique $\Lambda$-linear injective map

$$
\mathrm{FE}_{\Lambda}: \mathcal{S}\left(K^{p}, \Lambda\right) \hookrightarrow \Lambda\left[\left[q^{T}, T \in \mathcal{S}^{+}\right]\right] \widehat{\otimes} \mathcal{C}_{\mathbb{M}_{1}^{\prime}}
$$

such that for any admissible algebraic weight $\left(a^{\prime}, b^{\prime}\right), a^{\prime} \geqslant b^{\prime} \geqslant 0, k^{\prime}=a^{\prime}+3, \ell^{\prime}=b^{\prime}+3$, one has a commutative diagram

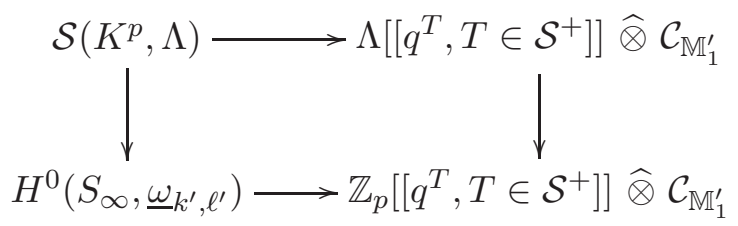

where the vertical maps are given by the reduction modulo $P_{a^{\prime}, b^{\prime}}$ and the bottom horizontal map is the classical q-expansion map.

We call $\mathrm{FE}_{\Lambda}$ the $\Lambda$-adic $q$-expansion map. We express the commutative diagram above by saying that the $\Lambda$-adic $q$-expansion map interpolates $p$-adically the classical $q$-expansion maps for nearly ordinary forms.

Proof. Let $R=\mathbb{Z}_{p}\left[\left[q^{T}, T \in \mathcal{S}^{+}\right]\right]$and $R_{\Lambda}=R \widehat{\otimes} \Lambda=\Lambda\left[\left[q^{T}, T \in \mathcal{S}^{+}\right]\right]$. Since $\mathcal{S}\left(K^{p}\right)$ is defined as a direct factor in the submodule of $\mathbb{N}^{\prime}$-invariants of $V_{\text {! }}$ the restriction of $\mathrm{FE}$ again yields an injection $\left(e V_{!}^{\mathbb{N}^{\prime}}\right)_{\mathfrak{m}} \hookrightarrow R \widehat{\otimes} \mathcal{C}_{\mathbb{M}_{1}^{\prime}}$ with flat cokernel.

The flatness of the cokernel implies the surjectivity of the dual map

$$
\operatorname{Hom}\left(R \widehat{\otimes} \mathcal{C}_{\mathbb{M}_{1}^{\prime}}, \mathbb{Z}_{p}\right) \widehat{\otimes} \Lambda \rightarrow \mathcal{S}^{*}\left(K^{p}\right) .
$$

For any $\Lambda$-module $M$, let us write $M^{\prime}$ for its $\Lambda$-dual. Dualizing over $\Lambda$ the surjection above, we obtain an injection of $\mathcal{S}\left(K^{p}, \Lambda\right)$ into the $\Lambda$-bidual $R_{\Lambda}^{\prime \prime}$ of $R_{\Lambda} \widehat{\otimes} \mathcal{C}_{\mathbb{M}_{1}^{\prime}}$

$$
\mathrm{FE}: \mathcal{S}\left(K^{p}, \Lambda\right) \hookrightarrow R_{\Lambda}^{\prime \prime}
$$

Let us show that it actually takes values in the submodule $R_{\Lambda}$ of $R_{\Lambda}^{\prime \prime}$. Notice first that $M=\operatorname{ImFE}$ is a finite free $\Lambda$-module. Consider the submodule $N=\operatorname{Im}(\mathrm{FE}) \cap \mathrm{R}_{\Lambda} \widehat{\otimes} \mathcal{C}_{\mathbb{M}_{1}^{\prime}}$. By induction on the rank of $M$, one sees easily that if they are not equal, there exists a prime ideal $P_{a^{\prime}, b^{\prime}, c^{\prime}}$ (for some $\left.\left(a^{\prime}, b^{\prime}, c^{\prime}\right), a^{\prime} \geqslant b^{\prime} \geqslant 0\right)$, such that $N+P_{a^{\prime}, b^{\prime}, c^{\prime}} M \neq M$. On the other hand, for any weight $\left(a^{\prime}, b^{\prime}\right)$, $a^{\prime} \geqslant b^{\prime} \geqslant 0$, we have by Hida [Hid02, Theorem 1.1(6)]:

$$
\mathcal{S}\left(K^{p}, \Lambda\right) / P_{a^{\prime}, b^{\prime}, c^{\prime}}=H^{0}\left(S_{\infty}, \underline{\omega}_{k^{\prime}, \ell^{\prime}}\right)_{\mathfrak{m}} \otimes \nu^{c^{\prime}}
$$

where $k^{\prime}=a^{\prime}+3$ and $\ell^{\prime}=b^{\prime}+3$. Moreover, the map FE modulo $P_{a^{\prime}, b^{\prime}, c^{\prime}}$ coincides with the usual $p$-adic $q$-expansion map

$$
H^{0}\left(S_{\infty}, \underline{\omega}_{k^{\prime}, \ell^{\prime}}\right) \hookrightarrow R \widehat{\otimes} \mathcal{C}_{\mathbb{M}_{1}^{\prime}}
$$

Hence, for any prime $P_{a^{\prime}, b^{\prime}} \in \operatorname{Spec} \Lambda$ as above, $\operatorname{Im}(\mathrm{FE}) \subset \mathrm{R}_{\Lambda} \widehat{\otimes} \mathcal{C}_{\mathbb{M}_{1}^{\prime}}+\mathrm{P}_{\mathrm{a}^{\prime}, \mathrm{b}^{\prime}} \mathrm{R}_{\Lambda}^{\prime \prime}$, that is, $M=$ $N+P_{a^{\prime}, b^{\prime}} N$ : a contradiction. 


\section{NeARLY ORDinARY RANK FOUR Galois REPRESENTATIONS}

\subsection{Specialization and $p$-adic limits}

We have shown in [GT05, Theorem 4.3.5] that all representations $\pi^{\prime}$ occurring in $H^{3}\left(S_{K^{p} \times I_{1}}\right.$, $\left.V_{a^{\prime}, b^{\prime}}(\mathbb{C})\right)_{\mathfrak{m}}$ are stable at $\infty$. Let $\theta_{\pi^{\prime}}: \mathbf{T}_{a^{\prime}, b^{\prime}} \rightarrow \mathcal{O}^{\prime}$ be the character associated to $\pi^{\prime}$. Then, the $\theta_{\pi^{\prime}}$-isotypical component of $H^{0}\left(S_{K^{p} \times I_{1}}, \underline{\omega}_{k^{\prime}, \ell^{\prime}}\right)_{\mathfrak{m}}$ does not vanish.

Let $\mathcal{K}$ be a finite extension of the field of fractions of the Hida-Iwasawa algebra $\Lambda$ and $\mathcal{O}_{\mathcal{K}}$ be the integral closure of $\Lambda$ in $\mathcal{K}$.

Definition 4.4. A $\Lambda$-adic eigensystem for $\mathbf{T}$ is a $\Lambda$-algebra homomorphism $\Theta: \mathbf{T} \rightarrow \mathcal{K}$. It corresponds to an irreducible component of $\operatorname{Spec} \mathbf{T}$. It takes values in $\mathcal{O}_{\mathcal{K}}$.

We generalize slightly the notion of admissible algebraic weight as the set of algebraic primes $\mathbb{P}$ of Spec $\mathcal{O}_{\mathcal{K}}$ such that $\mathbb{P} \cap \Lambda$ is admissible algebraic. Let us fix a $\Lambda$-adic eigensystem $\Theta$ such that there exists an algebraic prime $P$ above $P_{a, b}$ at which the specialization $\Theta_{\mathbb{P}}=\Theta(\bmod \mathbb{P})$ in weight $(a, b)$ corresponds to our original $\pi$. The existence of $\Theta$ is guaranteed by the going-down theorem for the flat extension $\Lambda \rightarrow \mathbf{T}$.

Let us specialize $\Theta$ at an algebraic prime $\mathbb{P}_{0}$ corresponding to a non-dominant algebraic weight $\left(a_{0}, b_{0}\right)$ with $a_{0} \geqslant b_{0}$, but such that $k_{0}=a_{0}+3$ and $\ell_{0}=b_{0}+3$ are non-negative. In our application $\left(a_{0}, b_{0}\right)$ will be $(-1,-1)$, but for the moment, this does not matter.

We have defined the module $\mathcal{S}\left(K^{p}, \Lambda\right)$ of nearly ordinary $\Lambda$-adic families of Siegel cusp forms; it is a finite free $\Lambda$-module and a finite $\mathbf{T}$-module.

\section{THEOREM 7.}

(1) The $\mathbf{T} \otimes_{\Lambda} \mathcal{K}$-module $\mathcal{S}^{*}\left(K^{p}\right) \otimes_{\Lambda} \mathcal{K}$ is free of rank $m$.

(2) There exists a false cusp form $f_{0} \in H^{0}\left(S_{\infty}, \underline{\omega}_{k_{0}, \ell_{0}}\right)$, which is an eigenform for the character $\Theta_{a_{0}, b_{0}}$.

(3) There exists a sequence of weights $\left(k_{i}, \ell_{i}\right)$ such that

$$
\left(k_{i}, \ell_{i}\right) \equiv\left(k_{0}, \ell_{0}\right) \quad\left(\bmod (p-1) t p^{i}\right)
$$

and a sequence of classical cusp forms

$$
f_{i} \in H^{0}\left(S_{\left.K^{p} \times K_{p}^{\max }, \underline{\omega}_{k_{i}, \ell_{i}}\right)}\right.
$$

whose q-expansions converge towards that of $f_{0}$.

Proof. Fix an arbitrary very regular dominant weight $\left(a^{\prime}, b^{\prime}\right)$. Let $k^{\prime}=a^{\prime}+3$ and $\ell^{\prime}=b^{\prime}+3$; let $K\left(P_{a^{\prime}, b^{\prime}}\right)$ be the residue field of $\Lambda$ at $P_{a^{\prime}, b^{\prime}}$. By Corollary $3.3 \mathbf{V} \otimes_{\Lambda} K\left(P_{a^{\prime}, b^{\prime}}\right)$ is dual to $\left(\rho_{\mathbf{T}_{a^{\prime}, b^{\prime}}} \otimes \mathbb{Q}_{p}\right){ }^{\oplus m}$ as Galois $\times$ Hecke-module; hence by the ordinarity at $p$ of $\rho_{\mathbf{T}_{a^{\prime}, b^{\prime}}}$ it admits a $\operatorname{Gal}\left(\overline{\mathbb{Q}}_{p} / \mathbb{Q}_{p}\right)$-stable fourstep filtration, whose last step $F^{+}$is free of rank $m$ over $\mathbf{T} \otimes_{\Lambda} K\left(P_{a^{\prime}, b^{\prime}}\right)$ (recall that $m$ denotes the multiplicity of $\pi$ ).

On the other hand, by Proposition 3.2, $\mathbf{V} \otimes_{\Lambda} K\left(P_{a^{\prime}, b^{\prime}}\right)$ is $\operatorname{Gal}\left(\overline{\mathbb{Q}}_{p} / \mathbb{Q}_{p}\right)$-isomorphic to $H^{3}$

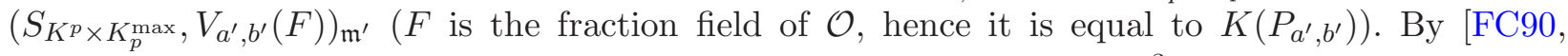

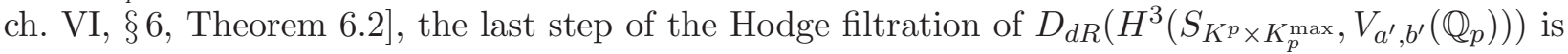

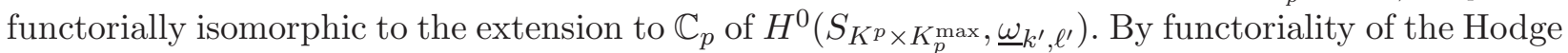
decomposition, a similar statement is true after localization at $\mathfrak{m}^{\prime}$. Using the fact that $\mathbf{V} \otimes_{\Lambda} K\left(P_{a^{\prime}, b^{\prime}}\right)$ is crystalline at $p$, one sees that $F^{+} \otimes \mathbb{C}_{p}$ is also the last step of the Hodge filtration, hence is canon-

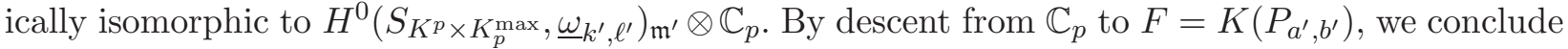

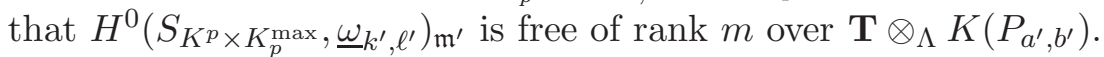

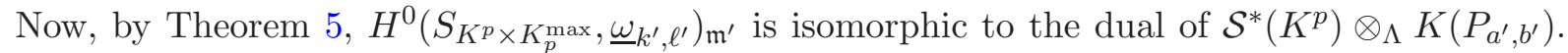
Moreover, by the same theorem, $\mathcal{S}^{*}\left(K^{p}\right)$ is free of finite rank over $\Lambda$. Hence, by Nakayama's lemma, $\mathcal{S}\left(K^{p}\right)^{*} \otimes_{\Lambda} \Lambda_{\left(P_{a^{\prime}, b^{\prime}}\right)}$ is free of rank $m$ over $\mathbf{T} \otimes_{\Lambda} \Lambda_{\left(P_{a^{\prime}, b^{\prime}}\right)}$. This proves part (1). 


\section{J. Tilouine}

(2) Recall that $\mathbf{T}$ is a reduced $\Lambda$-algebra by definition, hence $\mathbf{T} \otimes_{\Lambda} \mathcal{K}$ is semisimple. In particular, given our $\Lambda$-adic eigensystem $\Theta: \mathbf{T} \rightarrow \mathcal{K}$, there is a decomposition of $\mathbf{T}_{\mathcal{K}}=\mathbf{T} \otimes_{\Lambda} \mathcal{K}$ as a product of $K$-algebras $\mathbf{T}_{\mathcal{K}} \cong \mathcal{K} \times \mathbf{T}_{\mathcal{K}}^{\Theta}$, in which the first projection is given by $\Theta \otimes I d_{\mathcal{K}}$. We denote by $1_{\Theta}$ the idempotent of $\mathbf{T}_{\mathcal{K}}$ corresponding to the unit element of the first factor $\mathcal{K}$.

Let $\mathcal{S}\left(K^{p}, \mathcal{O}_{\mathcal{K}}\right)=\mathcal{S}\left(K^{p}, \Lambda\right) \otimes_{\Lambda} \mathcal{O}_{\mathcal{K}}$, respectively $\mathcal{S}\left(K^{p}, \mathcal{K}\right)=\mathcal{S}\left(K^{p}, \Lambda\right) \otimes_{\Lambda} \mathcal{K}$. Then $1_{\Theta}$ acts on $\mathcal{S}\left(K^{p}, \mathcal{K}\right)$ and we can form $\mathcal{S}\left(K^{p}\right)_{\Theta}=\mathcal{S}\left(K^{p}, \mathcal{O}_{\mathcal{K}}\right) \cap 1_{\Theta} \mathcal{S}\left(K^{p}, \mathcal{K}\right)$; it is a reflexive $\mathcal{O}_{\mathcal{K}}$-submodule of $\mathcal{S}\left(K^{p}, \mathcal{O}_{\mathcal{K}}\right)$. We call $\mathcal{S}\left(K^{p}\right)_{\Theta}$ the module of $\Theta$-eigen cusp forms over $\mathcal{O}_{\mathcal{K}}$. By tensoring FE by $I d_{\mathcal{K}}$ and restricting to the $\Theta$-eigenspace, we obtain

$$
\mathrm{FE}_{\Theta}: \mathcal{S}\left(K^{p}, \mathcal{O}_{\mathcal{K}}\right)_{\Theta} \hookrightarrow \mathcal{O}_{\mathcal{K}}[[q]]
$$

By Theorem $5, \mathcal{S}\left(K^{p}, \mathcal{O}_{\mathcal{K}}\right) \otimes \mathcal{O}_{\mathcal{K}} K\left(\mathbb{P}_{0}\right)$ is isomorphic to the localization $H^{0}\left(S_{\infty}, \underline{\omega}_{k_{0}, \ell_{0}}\right)_{\mathfrak{m}} \otimes F$. Let us prove that there exists $\mathfrak{f} \in \mathcal{S}\left(K^{p}\right)_{\Theta}$ whose reduction modulo $\mathbb{P}_{0}$ defines a non-zero element of the $\Theta_{\mathbb{P}_{0}}$-isotypical component of the space $H^{0}\left(S_{\infty}, \underline{\omega}_{k_{0}, \ell_{0}}\right)_{\mathfrak{m}} \otimes F$ of false cusp forms of weight $\left(k_{0}, \ell_{0}\right)$.

We have $\mathbb{P}+\varpi \mathcal{O}_{\mathcal{K}}=\mathbb{P}_{0}+\varpi \mathcal{O}_{\mathcal{K}}=\max \left(\mathcal{O}_{\mathcal{K}}\right)$ (here $\mathbb{P}$ is our given prime of weight $(a, b)$ ).

By assumption, there exists a $\Theta_{\mathbb{P}}$-eigenform $f^{\prime} \in \mathcal{S}\left(K^{p}, \mathcal{O}_{\mathcal{K}}\right) / \mathbb{P} \mathcal{S}\left(K^{p}, \mathcal{O}_{\mathcal{K}}\right)$ that is not divisible by $\varpi$. Now, the map

$$
\mathcal{S}\left(K^{p}\right)_{\Theta} \otimes_{\mathcal{O}_{\mathcal{K}}} K(\mathbb{P}) \rightarrow \mathcal{S}\left(K^{p}, \mathcal{O}_{\mathcal{K}}\right) \otimes_{\mathcal{O}_{\mathcal{K}}} K(\mathbb{P})
$$

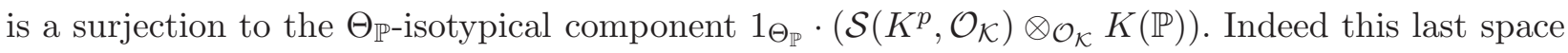
is the image of $1_{\Theta} \mathcal{S}\left(K^{p}, \mathcal{O}_{\mathcal{K}}\right)_{(\mathbb{P})}$, which is contained in the localization at $\mathbb{P}$ of $\mathcal{S}\left(K^{p}\right)_{\Theta}$.

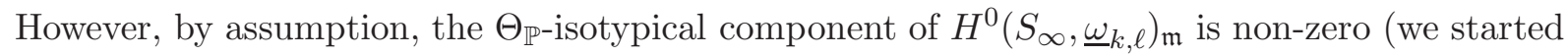
with a $\pi$ occurring in this space). Therefore, there exists $\mathfrak{f} \in \mathcal{S}\left(K^{p}\right)_{\Theta}$, which lifts a $\Theta_{\mathbb{P}}$-eigenform $f_{0}^{\prime} \in \mathcal{S}\left(K^{p}, \mathcal{O}_{\mathcal{K}}\right) / \mathbb{P} \mathcal{S}\left(K^{p}, \mathcal{O}_{\mathcal{K}}\right)$; one can assume that $\varpi$ does not divide $f_{0}^{\prime}$. Hence, $f_{0}^{\prime}$ is still not zero in the Theta-eigenspace of

$$
\mathcal{S}\left(K^{p}, \mathcal{O}_{\mathcal{K}}\right) / \max \left(\mathcal{O}_{\mathcal{K}}\right) \mathcal{S}\left(K^{p}, \mathcal{O}_{\mathcal{K}}\right)
$$

hence, the $\Theta_{\mathbb{P}_{0}}$-eigenspace of $\mathcal{S}\left(K^{p}, \mathcal{O}_{\mathcal{K}}\right) / \mathbb{P}_{0} \mathcal{S}\left(K^{p}, \mathcal{O}_{\mathcal{K}}\right)$ is non-zero; any lift $\mathfrak{f} \in \mathcal{S}\left(K^{p}\right)_{\Theta}$ of a non-zero element $f_{0}$ of this space answers the question.

We define the Hida's weight space $\mathcal{W}$ as the rigid $p$-adic space whose $\mathbb{C}_{p}$-points are canonically $\mathcal{W}\left(\mathbb{C}_{p}\right)=\operatorname{Hom}\left(\mathbb{T}, \mathbb{C}_{p}^{\times}\right)$. It is an open three-ball. Therefore, it is a wide open rigid space. Similarly, one can define the rigid space $\mathcal{X}_{\mathcal{K}}$ associated to $\mathcal{O}_{\mathcal{K}}$. It will come equipped with a finite flat rigid morphism to $\mathcal{W}$ corresponding to the structural morphism $\Lambda \rightarrow \mathcal{O}_{\mathcal{K}}$.

Consider the $q$-expansion of the $\Lambda$-adic cusp form $\mathfrak{f}_{1}$. Its coefficients define rigid analytic functions on $\mathcal{X}_{\mathcal{K}}$.

We define $f_{0}$ as the reduction of $\mathfrak{f}_{1}$ modulo $\mathbb{P}_{0}$. By definition, the $q$-expansions of the forms obtained by specialization of $\mathfrak{f}_{1}$ at algebraic weights do converge towards that of $f_{0}$. If we choose very regular weights, the corresponding forms are classical of level $K^{p} \times K_{p}^{\max }$ by [Hid02, Theorem 1.1(6)].

Apply the above consideration for specializations at very regular weights. This provides a sequence of classical cusp eigenforms of very regular weights, which can be chosen as in the statement of the control theorem, Theorem 2 , above, converging to $\left(k_{0}, \ell_{0}\right)$ and level prime to $p$ whose $q$-expansions converge to that of $f_{0}$.

\subsection{Overconvergence}

In the previous theorem, one conjectures that if the Galois representation associated to $\Theta_{a_{0}, b_{0}}$ does come from geometry (for instance, from an abelian variety for $\left(a_{0}, b_{0}\right)=(-1,-1)$ ), the $\Theta_{a_{0}, b_{0}}{ }^{-}$ eigenform $f_{0}$ is also classical, of level $K^{p} \times \Pi_{Q}$, where $\Pi_{Q}$ denotes the Siegel parahoric subgroup of $G\left(\mathbb{Z}_{p}\right)$. For the moment, as a first step towards this classicity conjecture, one can prove its overconvergence. The proof follows the same lines as in [BT99, Lemma 1]. 


\section{NeARLY ORDinARY RANK FOUR Galois REPRESENTATIONS}

Let $X^{\text {rig }}, X^{* \text {,rig }}, \bar{X}^{\text {rig }}$ be the rigid analytic space associated to the $p$-adic completion of its corresponding $\mathbb{Z}_{p}$-scheme. The rigid space $X^{\text {rig }}$ carries a rigid analytic principally polarized abelian variety, namely the rigid abelian family $A^{\text {rig }}$ associated to the $p$-adic completion of the universal abelian scheme $A \rightarrow X$. The universality of the rigid family $A^{\text {rig }} \rightarrow X^{\text {rig }}$ in the category of rigid abelian spaces is not known to the author. This property is not used in the sequel. Let $\mathcal{G}^{\text {rig }} \rightarrow \bar{X}^{\text {rig }}$ be the semiabelian rigid analytic group over $\bar{X}^{\text {rig }}$ obtained by the construction above from the semiabelian group scheme $\mathcal{G} \rightarrow \bar{X}$.

Let $X^{\text {rig,ord }}, X^{* \text {,rig,ord }}, \bar{X}^{\text {rig,ord }}$ be the rigid analytic open subdomain associated to the open formal subschemes $S_{\infty}, S_{\infty}^{*}, \bar{S}_{\infty}$ of $X, X^{* \text {,rig }}, \bar{X}^{\text {rig }}$, respectively. One has $X^{\text {rig,ord }}=\left\{x \in X^{\text {rig; }}\right.$ $\left.|E(x)|_{p} \geqslant 1\right\}$ and similarly for $X^{*, \text { rig,ord }}$.

By a theorem of Abbès and Mokrane [AM04, Proposition 8.2.3], the open subdomain $\mathcal{D}$ of $\bar{X}^{\text {rig }}$ defined as the locus where the lifting $E$ of the Hasse invariant satisfies $|E|_{p}>p^{-1 / p(p-1)}$ is endowed with a finite flat group scheme $C_{\text {can }}$ of rank $p^{2}$ whose restriction to the ordinary locus is canonically isomorphic to $A[p]^{0}$. For each $\left.r \in\right] p^{-1 / p(p-1)}, 1\left[\cap p^{\mathbb{Q}}\right.$, we define

$$
\mathcal{D}(r)=\left\{x \in \bar{X}^{\text {rig }}(L) ;|E|_{p} \geqslant r\right\} .
$$

These domains are admissible, quasi-compact relatively compact.

Note that if $r<r^{\prime}$ in $] p^{-1 / p(p-1)}, 1$, we have $\mathcal{D}\left(r^{\prime}\right) \subset \mathcal{D}(r)$ and for any finite extension $L$ of $\mathbb{Q}_{p}$ $X^{\text {rig,ord }}(L)=\bigcap_{r \in] p^{-1 / p(p-1)}, 1[} \mathcal{D}(r)(L)$ and $\mathcal{D}(L)=\bigcup_{r \in] p^{-1 / p(p-1)}, 1[} \mathcal{D}(r)(L)$.

We consider the $L$-vector space of overconvergent

$$
S_{\kappa}(K ; r)=H^{0}\left(\mathcal{D}(r) \times L, \underline{\omega}_{\kappa}\right)
$$

If $r$ is in $\left|L^{\times}\right|_{p}$, this is a Banach space for the norm $|f|=\sup _{x \in \mathcal{D}(r)(L)}|f(x)|_{p}$ by [BGR84, Theorem 4.1.6]. In particular, for any $r<r^{\prime}$ in $] p^{-1 / p(p-1)}, 1\left[\cap\left|L^{\times}\right|_{p}\right.$, the inclusions

$$
\operatorname{res}_{r, r^{\prime}}: S_{\kappa}(K ; r) \hookrightarrow S_{\kappa}\left(K ; r^{\prime}\right)
$$

are completely continuous by [KL05, Proposition 2.4.1].

It should be noted that for this proof as well as for the proofs of the following results of this section (the main result being the complete continuity of the operator $\left.U_{p, 1}\right)$, there is no need to assume that the weight $\kappa$ is cohomological (that is $k_{1} \geqslant k_{2} \geqslant 3$ ). We will indeed apply this to $\kappa=(2,2)$.

The canonical lifting $F_{\text {can }}$ of the Frobenius endomorphism is defined as a rigid morphism $\mathcal{D}(r) \rightarrow \bar{X}^{\text {rig }}$ by [AM04, Lemma 8.2.1].

Proposition 4.5. There exists $r \in] p^{-1 / p^{2}(p-1)}, 1\left[\cap p^{\mathbb{Q}}\right.$ such that $F_{\text {can }}$ maps $\mathcal{D}(r)$ into $\mathcal{D}\left(r^{p}\right)$ and is finite flat of degree $p^{3}$. It yields a continuous homomorphism of Banach space $\phi=F_{\text {can }}^{*}: S_{\kappa}\left(K ; r^{p}\right) \rightarrow$ $S_{\kappa}(K ; r)$ and a trace homomorphism $\operatorname{Tr}_{\phi}: S_{\kappa}(K ; r) \rightarrow S_{\kappa}\left(K ; r^{p}\right)$.

Proof. This is [KL05, Lemma 3.1.7]. Note that in its proof of this lemma, the assumption that $r \in] p^{-1 / p^{2}(p-1)}, 1\left[\cap p^{\mathbb{Q}}\right.$ is sufficiently close to 1 is needed, because use is made of the maximum modulus principle, which provides only the existence of some radius for which the maximum is reached. The complete continuity of $\operatorname{res}_{r^{p}, r}$ yields the following.

Corollary 4.6. There exists $r \in] p^{-1 / p^{2}(p-1)}, 1\left[\cap\left|L^{\times}\right|_{p}\right.$, the composition $\psi=\operatorname{res}_{r^{p}, r} \circ \operatorname{Tr}_{\phi}$ defines a completely continuous endomorphism of the Banach space $S_{\kappa}(K ; r)$.

There is an overconvergent $q$-expansion principle, which follows clearly from the connectedness of $\bar{X}^{\text {rig }}$ : the evaluation on the rigid Mumford family $\mathcal{G}_{\eta}^{\text {rig }} \rightarrow \mathcal{S}_{\eta}$ defines an injective $L$-linear homomorphism

$$
S_{\kappa}(K ; r) \hookrightarrow L\left[\left[q^{T} ; T \in \mathcal{S}^{+}\right]\right]
$$




\section{J. Tilouine}

We define $U_{p, 1}$ as $p^{-3} \psi$ be the operator corresponding to the weight $\kappa=(2,2)$. We denote by $S_{2}(K ; r)$ the $L$-Banach space of forms of weight $(2,2)$. Then the following corollary follows immediately from Corollary 4.6.

COROLlary 4.7. There exists $L$ sufficiently large and $r \in] p^{-1 / p^{2}(p-1)}, 1\left[\cap\left|L^{\times}\right|_{p}\right.$ such that the operator $U_{p, 1}$ is completely continuous on the Banach space $S_{2}(K ; r)$ of weight 2 overconvergent p-adic cusp forms.

Recall that by [Ser62, Proposition 7], one can define a Fredholm determinant $P(t)=\operatorname{det}\left(1-t U_{p, 1}\right)$ which is a $p$-adic entire function of $t$ and such that $\lambda \in \overline{\mathbb{Q}}_{p}$ is a non-zero eigenvalue of $U_{p, 1}$ if and only if $P\left(\lambda^{-1}\right)=0$; so that the non-zero eigenvalues of $U_{p, 1}$ form a sequence decreasing to 0 . By Proposition 12 and Remark 3 following this proposition in [Ser62], each spectral subspace associated to a non-zero eigenvalue is finite dimensional (its dimension being equal to the multiplicity of the root $\lambda^{-1}$ of $P$ ) and there is a direct sum decomposition of the Banach space as the sum of the (finitedimensional) spectral subspace and the largest closed subspace on which $U_{p, 1}-\lambda$ is invertible. In particular, the set of eigenvalues $\lambda_{i} \in \overline{\mathbb{Q}}_{p}$ of $U_{p, 1}$ such that $\operatorname{ord}_{p}\left(\lambda_{i}\right)=0$ is finite. Moreover, one has a direct sum decomposition of the Banach space $S_{2}(K, r)$ as $S_{2}(K, r)^{(0)} \oplus S_{2}(K, r)^{>0}$, where the first space is finite dimensional, defined as the direct sum of the spectral subspaces for all eigenvalues $\lambda_{i}$ with $\operatorname{ord}_{p}\left(\lambda_{i}\right)=0$, and the second is the (closed) largest subspace on which all of the operators $U_{p, 1}-\lambda_{i}$ are invertible.

We denote by $e_{1}$ the projector of $S_{2}(K ; r)$ onto $S_{2}(K, r)^{0}$ along $S_{2}(K, r)^{(>0)}$ associated to $U_{p, 1}$.

Corollary 4.8. The false cusp form $f_{0} \in H^{0}\left(S_{\infty}, \underline{\omega}_{2,2}\right)$, which is eigen for the character $\Theta_{-1,-1}$, is overconvergent (of weight 2 ) on the domain $\mathcal{D}(r)$ for some $r \in] p^{-1 / p^{2}(p-1)}, 1\left[\cap\left|L^{\times}\right|_{p}\right.$.

Proof. We have noted that there exists a sequence $\left(g_{i}\right)$ of ordinary classical Siegel cusp forms of weights $\kappa_{i}=\left(k_{i}, k_{i}\right)\left(k_{i}=2+(p-1) t p^{i}\right)$ and level $K$ (prime-to- $\left.p\right)$, with coefficients in a fixed finite extension $L$ of $\mathbb{Q}_{p}$, whose $q$-expansions converge to that of $f_{0}$. In particular, for any $r \in] p^{-1 / p^{2}(p-1)}, 1\left[\cap\left|L^{\times}\right|_{p}\right.$, for any $i \geqslant 1, g_{i}$ belongs to $S_{\kappa_{i}}(K ; r)$, hence $e_{1}\left(g_{i} / E^{t p^{i}}\right)$ belongs to the subspace $S_{2}(K ; r)^{(0)}$ of $S_{2}(K ; r)$ on which the valuation of the eigenvalues of $U_{p, 1}$ is 0 . As noted above, by complete continuity of $U_{p, 1}$, the subspace $S_{2}(K ; r)^{(0)}$ is finite dimensional over $L$. Therefore, it is complete for any norm and, in particular, for the norm defining the topology of convergence of the $q$-expansions. The sequence of $q$-expansions of $e_{1}\left(g_{i} / E^{t p^{i}}\right)$ still converges to $f_{0}$ because $E^{t p^{i}} \equiv 1\left(\bmod p^{i+1}\right)$. Hence, by the $q$-expansion principle, $f_{0} \in S_{2}(K ; r)^{(0)}$.

\section{Abelian surfaces}

Let $p \geqslant 5$ be a prime, and $\alpha+\beta<p-1$; let $N$ be a square-free integer prime to $p$. Let $A$ be an abelian surface defined over $\mathbb{Q}$ with semistable bad reduction at primes dividing $N$, and which acquires good ordinary reduction over the field $\mathbb{Q}\left(\zeta_{p}\right)$ generated by $p$ th roots of unity. Let us assume it has a polarization of degree prime to $p$ (for instance, a principal polarization). Let $S$ be the set of primes dividing $N p$. The action of the Galois group $\operatorname{Gal}(\overline{\mathbb{Q}} / \mathbb{Q})$ on the $p$-adic Tate module of $A$ defines an $S$-ramified symplectic rank four Galois representation $\rho_{A, p}: \operatorname{Gal}(\overline{\mathbb{Q}}) \rightarrow G\left(\mathbb{Z}_{p}\right)$. If $\ell$ divides $N$, one knows by the Néron-Ogg-Shafarevich-Grothendieck theorem that the image of the inertia group $I_{\ell}$ is unipotent of order 2 . Hence it is generated by a conjugate of $\epsilon_{2}$, if the reduction is purely toric, or a conjugate of $\eta_{2}$, if the reduction is an extension of an elliptic curve by a torus (for the notation, see the remark following Definition 1.3). Let $\bar{\rho}_{A, p}$ be the reduction modulo $p$ of $\rho_{A, p}$. In this setting, we make the following definition. 


\section{NEARLY ORDinARY RANK FOUR Galois REPRESENTATIONS}

Definition 5.1. We say that $\rho_{A, p}$ is $N$-good if it is semistable outside $p$, unramified outside $N p$, and for each prime $\ell$ dividing $N$, the image of the restriction of $\bar{\rho}_{A, p}$ to $I_{\ell}$ is generated by a conjugate of $\epsilon_{2}$.

Note that it implies that the same holds for $\rho_{A, p}$; therefore it implies that $A$ has purely toric reduction at each prime $\ell$ dividing $N$.

Let $\epsilon$ be the $p$-adic cyclotomic character and $\omega$ the Teichmüller lift of the cyclotomic character modulo $p$.

The restriction to the subgroup $I_{p}$ of inertia at $p$ of $\rho_{A, p}$ is provided by the following.

Lemma 5.2. The restriction of $\rho_{A, p}$ to $I_{p}$ is conjugated in $\operatorname{GSp}\left(4, \mathbb{Z}_{p}\right)$ to

$$
\left(\begin{array}{cccc}
\epsilon \omega^{\alpha+\beta} & * & * & * \\
0 & \epsilon \omega^{\alpha} & * & * \\
0 & 0 & \omega^{\beta} & * \\
0 & 0 & 0 & 1
\end{array}\right) \otimes \omega^{-(\alpha+\beta) / 2}
$$

for a suitable pair $(\alpha, \beta)$ of integers modulo $p-1$ not both 0 .

Proof. Since $A$ acquires good ordinary reduction over $\mathbb{Q}\left(\zeta_{p}\right)$, the restriction of $\rho_{A, p}$ to the inertia group $I\left(\overline{\mathbb{Q}}_{p} / \mathbb{Q}\left(\zeta_{p}\right)\right)$ leaves stable the connected-étale decomposition of $T_{p}\left(A \times \mathbb{Q}\left(\zeta_{p}\right)\right)$

$$
0 \rightarrow T_{p} A^{0} \rightarrow T_{p} A \rightarrow T_{p} A^{\text {ét }} \rightarrow 0 .
$$

Moreover, the Galois group $\operatorname{Gal}\left(\mathbb{Q}_{p}\left(\zeta_{p}\right) / \mathbb{Q}_{p}\right)$ also leaves this decomposition stable (it acts by geometric monodromy on $T_{p} A^{\text {ét }}$ and preserves $T_{p} A^{0}$ by duality). If one diagonalizes $\operatorname{Gal}\left(\mathbb{Q}_{p}\left(\zeta_{p}\right) / \mathbb{Q}_{p}\right)$ on $T_{p} A^{\text {ét }}$, one obtains two characters $\omega^{i_{3}}$ and $\omega^{i_{4}}$ giving its action. The characters $\omega^{i_{1}}$ and $\omega^{i_{2}}$ giving its action on $T_{p} A^{0}$ are necessarily such that (after reordering) $i_{1}+i_{4}=i_{2}+i_{3}=0$, since the similitude factor is known to be $\epsilon$. We put $\alpha=i_{1}+i_{2}$ and $\beta=i_{1}-i_{2}$. Note that these integers cannot be both 0 modulo $p-1$. Then, $i_{1}=(\alpha+\beta) / 2, i_{2}=(\alpha-\beta) / 2, i_{3}=(-\alpha+\beta) / 2$ and $i_{4}=-(\alpha+\beta) / 2$ and the result follows.

Definition 5.3. We say that $\bar{\rho}_{A, p}$ has $p$-limited weights or that the pair $(\alpha, \beta)$ is $p$-limited if $0<\beta \leqslant \alpha$ and $\alpha+\beta<p-1$.

For instance, the pair $(\alpha, \beta)=(1,1)$ is $p$-limited if $p \geqslant 5$; in this case, the strict ordinarity condition is not satisfied on the inertia subgroup $I_{p}: \bar{\chi}_{i} / \bar{\chi}_{i+2} \mid I_{p}=\omega(=1,2)$ (but it may be satisfied on $\left.D_{p}\right)$.

Definition 5.4. We say that $\bar{\rho}_{A, p}$ is strictly ordinary at $p$ if the characters $\bar{\chi}_{i}(i=1, \ldots, 4)$ on the diagonal of the restriction of $\bar{\rho}_{A, p}$ to the decomposition group at $p$ satisfy $\bar{\chi}_{i} / \bar{\chi}_{i+1} \notin\{1, \omega\}(i=1,2)$.

It implies a similar statement for $\rho_{A, p}$ with $\epsilon$ instead of $\omega$.

Definition 5.5. If $\rho_{A, p}$ is $N$-good, has $p$-limited weights, we say it is minimal modular if there exists a Siegel cusp form $g$ of weight $(k, \ell)$ with $k=\alpha+2$ and $\ell=\beta+2$ of level $N$ for the Klingen parahoric, such that the contragredient $\bar{\rho}_{A, p}^{\vee}$ of $\bar{\rho}_{A, p}$ is isomorphic to the reduction of $\rho_{g, p}$.

For $p, N$ as above, and for any $p$-limited pair $(\alpha, \beta)$, we introduce the set $\mathcal{A}_{p, \alpha, \beta ; N}$ of isomorphism classes of abelian surfaces defined over $\mathbb{Q}$, such that $\operatorname{Im} \bar{\rho}_{A, p}=G\left(\mathbb{F}_{p}\right), \rho_{A, p}$ is $N$-good, $A$ acquires good ordinary reduction over $\mathbb{Q}\left(\zeta_{p}\right)$, is strictly ordinary with $p$-limited weights given by the $p$-limited pair $(\alpha, \beta)$.

Theorem 8. For any $A \in \mathcal{A}_{p, \alpha, \beta ; N}$ strictly ordinary at $p$ and minimal modular, there exists a finite extension $\mathcal{O}$ of $\mathbb{Z}_{p}$ and an overconvergent Siegel cusp form $f_{A}$ of weight $(2,2)$ such that $\rho_{A, p}^{\vee}$ is conjugate to $\rho_{f_{0}, p}$ in $G(\mathcal{O})$. 


\section{J. Tilouine}

This theorem follows from the fact that the representation $\rho_{A, p}^{\vee}$ is an $N$-semistable $p$-ordinary deformation of $\bar{\rho}_{g, p}$. Therefore, the assumptions of [GT05, Theorem 2.2.2] are fulfilled, and hence Theorem 4 and Corollary 4.8 above apply.

Remark. It would be very interesting to produce numerical examples of $\mathcal{A}_{p, \alpha, \beta ; N} \neq \emptyset$. For instance, Poonen has checked that $\mathcal{A}_{13, \alpha, \beta ; 21739} \neq \emptyset$ (with $N=21739$ a prime) for a pair $(\alpha, \beta)$ that is unfortunately not 13-limited. Brumer (private communication) produced also several examples of smaller conductor, with pairs that might be $p$-limited. One would then need to find a Siegel cusp form of weight $(3,3)$ and level $N$ with Hecke eigenvalues matching the characteristic polynomial of Frobenius substitutions at many primes $q$. One is prevented from doing so for the moment because of the size of the calculations involved. So unfortunately no concrete application of the theorem is to be given at this moment. However, there is hope of relaxing the assumption on the full image to permit other cases of not too small images. We hope to come back to this in another work.

As a conclusive remark, one should mention that the Langlands conjecture implies that there exists a set $I$ and two lists $\left(T_{i}\right)_{i \in I}$ and $\left(T_{i}^{\prime}\right)_{i \in I}$, where:

(1) for each $i, T_{i}$ is a septuple $\left(G, X, r, \pi_{\infty}, P, F, E\right)$ consisting of a reductive group $G / \mathbb{Q}$ admitting a Shimura variety $S$ locally isomorphic to the hermitian symmetric domain $X$, a representation $r$ of the $L$-group of $G$, an admissible representation $\pi_{\infty}$ of $G(\mathbb{R})$ occurring in the coherent cohomology of $S$, a minimal parabolic subgroup $P$ of $G(\mathbb{A})$ and number fields $F$ and $E$;

(2) $T_{i}^{\prime}$ is a PEL type of submotives with coefficients in $E$ of abelian varieties with polarization and endomorphism defined over $F$;

such that for each $i \in I$ and for $T_{i}=\left(G, X, r, \pi_{\infty}, P, F, E\right)$, the set of Galois representations of $\bar{F} / F$ associated to the cuspidal representations $\pi$ of $G(\mathbb{A})$, with infinity type $\pi_{\infty}, P$-parahoric level and coefficients in $E$ is conjecturally identical to that associated to the submotives defined over $F$ with coefficients in $E$ of abelian varieties of type $T_{i}^{\prime}$.

We can quote several items of the list. In the first two examples, the representation of the $L$-group is the standard representation, and for the symplectic case, it is the spin representation.

- $T_{1}=\left(\mathrm{GL}_{2}, \mathcal{Z}_{1}, \pi_{2}, B, \mathbb{Q}, \mathbb{Q}\right)$ where $\mathcal{Z}_{1}$ is the upper half-plane and $\pi_{2}$ is the discrete series of weight 2 and $B$ is the upper triangular Borel subgroup of $\mathrm{GL}_{2}$. On the other hand, $T_{1}^{\prime}$ is the set of elliptic curves defined over $\mathbb{Q}$. Then the conjectured bijection has been established by Wiles [Wi195], Taylor and Wiles [TW95] and Breuil, Conrad, Diamond and Taylor [BCDT01].

- $T_{2}=\left(\operatorname{Res}_{\mathbb{Q}}^{F} \mathrm{GL}_{2}, \mathcal{Z}_{F}, \pi_{(2,2, \ldots)}, B, F, \mathbb{Q}\right)$ where $F$ is a totally real field $\mathcal{Z}_{F}=\mathcal{Z}_{1}^{I_{F}}$ is the Hilbert half-space $\left(I_{F}\right.$ denotes its set of embeddings into $\left.\mathbb{R}\right), \pi_{(2,2, \ldots)}$ is the discrete series of weight $(2,2, \ldots)$ and $B$ is the restriction of scalars of the upper triangular Borel subgroup of $\mathrm{GL}_{2}$ over $F$, while $T_{2}^{\prime}$ is the set of elliptic curves defined over $F$. This case of the conjecture has been established for many elliptic curves by Fujiwara (unpublished).

- $T_{3}=\left(\operatorname{GSp}(4), \mathcal{Z}_{2}, \pi_{(2,2)}, B, \mathbb{Q}, \mathbb{Q}\right)$ where $\mathcal{Z}_{2}$ is the Siegel half-space of genus $2, \pi_{(2,2)}$ is in the limit of discrete series of weight $(2,2)$ and $B$ is the standard Borel of $\operatorname{GSp}_{4}(\mathbb{Q}) ; T_{2}^{\prime}$ is the set of irreducible polarized abelian surfaces defined over $\mathbb{Q}$.

If one considers a real quadratic field $F$ and an abelian surface $A$ of type $T_{2}^{\prime}$ admitting multiplication by $F$ (such an abelian variety cannot be principally polarized), it is easy to see that another case of Fujiwara's theorem mentioned above (it applies under certain extra assumptions on the $p$-adic Galois representation $\rho_{A, p}$ of $A$ ) provides a Siegel cusp form of type $T_{3}$. Indeed, $\rho_{A, p}$ is induced from a representation $\rho$ of $\operatorname{Gal}(\bar{F} / F)$. By Fujiwara's theorem, $\rho$ comes from a Hilbert cusp form of weight $(2,2)$. The Theta lift of this form to $\mathrm{GSp}_{4}(\mathbb{Q})$ (due to Yoshida [Yos80] and Roberts [Rob01]) provides the desired Siegel cuspidal representation of infinity type $\pi_{(2,2)}$. 


\title{
NeARLY ORDinARY RANK FOUR Galois REPRESENTATIONS
}

If we consider generic abelian varieties of type $T_{3}^{\prime}$, Theorem 4 of the present paper provides evidence for the correspondence.

A further conjectural item of the list, involving jacobians of certain genus three curves, is detailed in the appendix to this paper by Blasius.

\section{ACKNOWLEDGEMENTS}

Part of this paper was written during visits at Nagoya University and at CalTech. The excellent working conditions in these institutions were appreciated. The author wishes to express his thanks to K. Fujiwara and D. Ramakrishnan for their invitation. Discussions with H. Hida, A. Mokrane and D. Blasius were also very useful. Finally, the author is also indebted to the referee whose careful reading helped to clarify several points.

\section{Appendix. A rank 3 generalization of the conjecture of Shimura and Taniyama}

\author{
Don Blasius
}

The conjecture of Shimura and Taniyama is a special case of a general philosophy according to which a motive of a certain type should correspond to a special type of automorphic forms on a reductive group. Now familiar extensions of the conjecture include (i) essentially the same statement for elliptic curves over totally real fields and (ii) the statement that for each irreducible abelian surface over $\mathbf{Q}$ there is a genus 2 Siegel modular holomorphic cusp form of weight 2 with the same (degree 4 ) $L$-function. Our purpose here is to give a new rank 3 variant of the Shimura-Taniyama conjecture in which elliptic curves are replaced by a naive analogue, the Picard curves. These curves have recently been much studied over finite fields, but their arithmetic study in characteristic zero has not yet attracted much interest. The main article to this appendix treats at length the case (ii) above.

\section{Motives}

Let $K$ be a number field and let $M$ be an irreducible motive defined over $K$ with coefficients in $\overline{\mathbf{Q}}$. We recall that this means that, for any rational prime $l$, the $l$-adic realization $M_{l}$ of $M$ is a $\overline{\mathbf{Q}_{l}}$-direct summand of a Tate-twist $H_{l}^{j}\left(X, \overline{\mathbf{Q}_{l}}\right)(k)$ of the $l$-adic cohomology $H_{l}^{j}\left(X, \overline{\mathbf{Q}_{l}}\right)$ of a smooth projective variety $X$ defined over $K$. Now, if $M$ has rank $N$, then it is standard to conjecture that there exists a cuspidal automorphic representation $\pi$ of $\operatorname{GL}\left(N, \mathbf{A}_{K}\right)$ such that, at each place $v$ of $K$, the local parameters, which are isomorphism classes of $N$-dimensional representations of Weil(-Deligne) group of $K_{v}$, associated to $\pi$ and $M$ coincide. This is often stated in the weaker form: $\pi$ and $M$ have the same $L$-function. Conversely, one hopes that all $\pi$, such that $\pi_{\infty}$ is algebraic [Clo90], so arise.

It is natural to refine the problem and ask: for which $M$ (and $K$ ) should there exist a Shimura variety $S h$ defined over $K$ such that $M$ (respectively $M_{l}$ ) is a direct summand, or even sub-quotient, of the cohomology motive $H^{*}(S h, \overline{\mathbf{Q}})$ of $S h$ (respectively of the $l$-adic cohomology $H^{*}\left(S h, \overline{\mathbf{Q}_{l}}\right)$ of $S h$ ). In general, the response to this question is quite subtle. In fact, it cannot be given without a detailed knowledge of cohomological A-packets, endoscopy, the representation $r$ employed to compute the Hasse-Weil zeta function and a host of local considerations, and to date all of these elements have been employed in examples. Even in the case where $M$ is a rank 2 submotive of $H^{1}(A)$ for $A$ an 


\section{J. Tilouine}

abelian variety over a totally real field $F$ is complex: while the $L$-functions of such motives should coincide with the $L$-functions of holomorphic Hilbert modular cusp forms of weight 2 , it is known that some such motives do not themselves occur in $H^{1}$ of any Shimura variety. (The question of whether all such $M$ occur non-effectively as Tate twists of factors of higher cohomology of Shimura varieties remains open [BR93].)

The requirement that $M$ be found in the cohomology of some $S h$ is unnecessarily strong. Indeed, the study of the case of modular forms of weight one, initiated in 1974 by Deligne and Serre [DS74], shows that one can sometimes make progress on the correspondence without this condition. This case and that of the preceding paper suggest that one may hope to establish the correspondence more generally in cases where $M$ should be associated to a $\pi$ having an algebraic $\pi_{\infty}$ that is holomorphic. However, a general description from the motivic viewpoint, i.e. in terms of varieties or even of Hodge structures, of this class of cases does not seem easy. Instead, our goal is to state an open case of this type for submotives of rank 3 of $H^{1}(A)$ for an abelian variety $A$. It will suffice here to let $K$ be a quadratic imaginary number field. Everything we do can be extended in a standard way to the more general case where $K$ is a CM field, but a general formulation would only belabor the ideas. Let $T$ be a CM field containing $K$, and $A$ be an abelian variety, defined over $K$, endowed with an action of an order in $T$, all of whose elements act $K$-rationally on $A$, and such that $1 \in T$ acts on $A$ as the identity map. Then the topological cohomology $H_{B}^{1}(A, \mathbf{Q})$ of $A(\mathbf{C})$ is a $T$ vector space; suppose that

$$
\operatorname{dim}_{T}\left(H_{B}^{1}(A, \mathbf{Q})\right)=3
$$

The tangent space $\operatorname{Tan}(A)$ of $A$ is a $K$ vector space of dimension $3 g$ where $[T: K]=g$. Then $\operatorname{Tan}(A)$ is naturally a $T \otimes_{\mathbf{Q}} K$-module. Let $J_{K}=\{1, \rho\}$ be the set of complex embeddings of $K$. Let $J_{T}$ be the set of complex embeddings of $T$. Then $J_{T}=J_{T}^{1} \cup J_{T}^{\rho}$ where $J_{T}^{1}$ is all the embeddings that restrict to 1 on $K$ and $J_{T}^{\rho}$ is all of the embeddings that restrict to $\rho$ on $K$. We now assume the representation $\Phi$ of $T$ on $\operatorname{Tan}(A)$ is equivalent to the following:

$$
\Phi \cong 2 \sum_{\sigma \in J_{T}^{1}} \sigma \oplus \sum_{\sigma \in J_{T}^{\rho}} \sigma .
$$

Then $H_{B}^{1}(A, \overline{\mathbf{Q}})$ is a three-dimensional $T \otimes \overline{\mathbf{Q}}$-vector space, and it is canonically decomposed as a direct sum:

$$
H_{B}^{1}(A, \overline{\mathbf{Q}})=\bigoplus_{\sigma \in J_{T}} H_{B}^{1}(A)_{\sigma}
$$

where $H_{B}^{1}(A)_{\sigma}$ is the $\overline{\mathbf{Q}}$-subspace of $H_{B}^{1}(A, \overline{\mathbf{Q}})$ on which $T$ acts via the embedding $\sigma$. Then $H_{B}^{1}(A)_{\sigma}$ is the realization in Betti (singular) cohomology of a motive $M_{\sigma}$ which is defined over $K$ and has coefficients in $\overline{\mathbf{Q}}$.

Note that, in general for irreducible motives with coefficients in a CM field $T$, the $T$-linear dual $\operatorname{Hom}_{T}(M, \mathbf{Q})$ is $T$-linearly isomorphic to the motive $M^{[\rho]}(w)$. Here the operation $[\rho]$ leaves the motive itself unchanged but alters the coefficient structure by precomposing with the automorphism $\rho: T \rightarrow T$ and the $(w)$ denotes the $w$-fold motivic Tate twist, where $w$ is the weight of $M$.

Examples of such motives arise as follows from the Picard curves, currently much studied in cryptography. Let

$$
Y^{3}=X^{4}+a X^{3}+b X^{2}+c X+d,
$$

where $a, b, c, d$ are in $\mathbf{Q}$, be the affine equation of a non-singular projective curve $C$. Then $C$ is called a Picard curve, has genus 3 and, after base change to $K=\mathbf{Q}\left[\zeta_{3}\right]$, the group of 6th roots of unity acts on $C$ via automorphisms. Hence, the $\operatorname{ring} \mathbf{Z}\left[\zeta_{3}\right]$ acts on the Jacobian $\operatorname{Jac}(C)$. Thus, the motive $H^{1}(C) \cong H^{1}(\operatorname{Jac}(C))$ is defined over $K$ and has coefficients in $T=K$. The associated decomposition of $H^{1}(C) \otimes \overline{\mathbf{Q}}$ into the direct sum of $H^{1}(C)_{1}$ and $H^{1}(C)_{\rho}$ is already defined over $K$. 


\section{NEARLY ORDINARY RANK FOUR GALOIS REPRESENTATIONS}

A calculation of a basis of the holomorphic differential forms on $C$ (see [Hol95]) shows that $T$ acts on $\operatorname{Tan}(\operatorname{Jac}(C))=H^{0}\left(C, \Omega^{1}\right)$ by

$$
\Phi=1+1+\rho
$$

Hence, $M_{1}=H^{1}(C)_{1}$ is of the class considered above. Note that the complex conjugate $C^{\rho}$ of $C$ is isomorphic to $C$. This means that

$$
M^{\rho} \cong M^{[\rho]}
$$

In view of the above, this means that

$$
M^{\vee} \cong M^{\rho}(w)
$$

where the ${ }^{\vee}$ denotes the $T(=K)$-linear dual. In particular, for a prime $l$, we have the relation of $l$-adic representations of $\operatorname{Gal}(\bar{K} / K)$ :

$$
M_{1, l}^{\vee} \cong M_{1, l}^{\rho}(w)
$$

\section{Automorphic forms}

Now let $V$ be a three-dimensional $K$-vector space endowed with an Hermitian form $H: V \times V \rightarrow K$. Define, as usual, the associated unitary similitude group

$$
G=\{g \in \mathrm{GL}(V, K) \mid H(g v, g w)=\lambda(g) H(v, w) \text { for all } v, w \in V\},
$$

where $\lambda(g) \in \mathbf{Q}$ is independent of $v$ and $w$. Then $G$ is a reductive algebraic group defined over $\mathbf{Q}$. Suppose that $H$ is chosen so that $G$ is quasi-split. We consider as usual the automorphic forms on the associated adelic groups $G\left(\mathbf{A}_{\mathbf{Q}}\right)$. The Shimura variety associated to $G$ is a family of surfaces [Shi63, Hol95]; the zeta functions of these surfaces were computed in [LR92]. The result is a formula that computes, for each cuspidal automorphic representation $\pi$ of $G\left(\mathbf{A}_{\mathbf{Q}}\right)$, an $L$-function $L(\pi, s)$ that has (unramified) Euler factors of degree 3. (See [BR94, BR92], and [LR92] for discussions and formulae that arise in the Hasse-Weil zeta function computations. In [BR92] the formula is stated without reference to the Hecke theory of $G$, using the base change [Rog90] of $\pi$ from $G^{1}$ to $G \times K=$ $\mathrm{GL}(3, K) \times \mathrm{GL}(1, K)$. This is convenient for exposition, but perhaps misleading in general.) In any case, the specific form of the construction is not important here.

Conjecture. Let $M_{l}$ be an irreducible three-dimensional $l$-adic representation of $\operatorname{Gal}(\bar{K} / K)$ that occurs as a subquotient of the cohomology in degree $\mathrm{w}$ of a variety defined over $K$. Suppose that

$$
M_{l}^{\vee} \cong M_{l}^{[\rho]}(\mathrm{w}) \text {. }
$$

Then there exists an $L$-packet $\Pi$ of cuspidal automorphic representations $\pi$ of $G\left(\mathbf{A}_{\mathbf{Q}}\right)$ such that, for almost all places $v$ of $K$

$$
L_{v}(\pi, s)=L_{v}\left(M_{l}, s\right) .
$$

Furthermore, at $\infty$, the $L$-packet $\Pi_{\infty}$ is that defined by an extension to ${ }^{L} G$ of the homomorphism $\mathbf{C}^{*}=W_{\mathbf{C}} \rightarrow \mathrm{GL}(3, \mathbf{C})$ defined by the Hodge structure of $M$.

This conjectural correspondence takes a $M_{l}$ with regular Hodge numbers, or regular Hodge-Tate weights, where regular means that all such weights occur with multiplicity at most one, to a $\Pi$ that is discrete series at $\infty$. It is not hard to see that $\Pi$ is unique if it exists. The converse result is a theorem: if $\Pi$ is an $L$-packet on $G\left(\mathbf{A}_{\mathbf{Q}}\right)$ that is discrete series at infinity, then there exists such an $M_{l}$; in fact, $M_{l}$ occurs in the cohomology of a fiber system of abelian varieties over the base $S h$, and a multiple of it is the $l$-adic realization of a submotive there. (This follows easily from the method of [BR93].)

While stated only at almost all places, it is not hard to complete the conjecture at the remaining places. The simplest way to do this is via the base change $B C(\Pi)$ of $\Pi$ to $\mathrm{GL}\left(3, \mathbf{A}_{K}\right) \times G L\left(1, \mathbf{A}_{K}\right)$. As usual, the statement at a finite place $v$ is just that the class of representations of the Weil-Deligne group of $K_{v}$ defined by the $M_{l}$, supposed to be all isomorphic, and that defined via $B C(\Pi)_{v}$ using 


\section{J. Tilouine}

the local Langlands correspondence and the representation of $\operatorname{GL}(3, \mathbf{C}) x \mathrm{GL}(1, \mathbf{C})$ used to form the $L$-function are the same.

If $M_{l}$ has two Hodge-Tate weights the same, but not all three the same, then under the conjectural correspondence the associated $L$-packet $\Pi$ consists of $\pi$ that are non-degenerate limits of discrete series at infinity. There are two such representations in each such archimedian packet and one is holomorphic. Further, $\Pi$ is stable, since $M_{l}$ is irreducible, and hence there exists a $\pi \in \Pi$ that is holomorphic at infinity. The structure of these archimedian $L$-packets is briefly reviewed in [Rog90, Section 12.3]. In the notation of that book, the archimedian $L$-parameter of $\Pi$ is determined by a triple $(a, b, c)$ of integers, in which $a \geqslant b \geqslant c$, and the case in question is that where either $a=b$ or $b=c$ but not both. These integers are related to the Hodge-Tate weights of $M_{l}$ by a simple rule; in particular, for the case $a=0, b=c=-p$ with $p>0$, then these numbers are $p, p$ and 0 .

Using the notation of [Rog90, Section 12.3], we can now link the previous discussion to this one by specializing the above general conjecture.

Conjecture. Let $M=H^{1}(A)$ be a motive of the type discussed above. Suppose that $M$ satisfies (*). Then there exists a cuspidal automorphic representation $\pi$ of $G$ such that

$$
\pi_{\infty}=J_{\phi}^{+},
$$

where $\phi=\phi(a, b, c)$ with $a=b=1$ and $c=0$, such that, at almost all places $v$ of $K$,

$$
L_{v}(M, s)=L_{v}(\pi, s) \text {. }
$$

Remarks.

(1) Here $J_{\phi}^{+}$is the lowest holomorphic limit discrete series representation of $G(\mathbf{R})$. In classical language, the automorphic forms in question are of weight 1 relative to the scalar factor of automorphy denoted by $\mu$ in [Shi78].

(2) As for classical forms of weight 1, the holomorphic vectors in these representations do not have an interpretation in terms of the cohomology of local systems on $S h$. Thus, one may expect to construct Galois representations attached to these forms only by congruences to forms of higher weight.

(3) It is easy to construct endoscopic examples of these forms if one relaxes the restriction that the motives be irreducible. For example, if $E$ is any elliptic curve $\mathbf{Q}$, then the product $E^{2}$ has endomorphisms $M_{2}(\mathbf{Z})$. Given another curve $E_{\mathrm{CM}} / \mathbf{Q}$ having (normalized) CM by $K$ over $K$, the $H^{1}$ of the three-fold $E^{2} \times E_{\mathrm{CM}}$ is the type of our conjecture. Since the Shimura-Taniyama Conjecture is now a theorem, an easy construction using the results of [Rog90] provides the sought $\pi$. More generally, if one starts with the motive attached to an holomorphic elliptic modular newform of weight at least 2, one can perform this construction. It is not hard to see that in this case the conjecture holds (in the sense of the local-global compatibility) at all places.

(4) It seems additional conjectural correspondences of this type, i.e. in which motives occurring in $H^{1}$ of abelian varieties are related to holomorphic forms, are very few. We hope to complete the list in a later publication.

Finally, as a further specialization, despite the redundancy, we state the conjecture for the Picard curves alone.

Conjecture (Picard curve case). Let $C$ be a Picard curve. Define $M_{1}=H^{1}(C)_{1}$ as above. Then there exists an automorphic representation $\pi$ of $G$ such that

$$
\pi_{\infty}=J_{\phi}^{+},
$$

where $\phi=\phi(a, b, c)$ with $a=b=1$ and $c=0$, such that, at almost all places $v$ of $K$,

$$
L_{v}(M, s)=L_{v}(\pi, s) \text {. }
$$




\section{NEARLY ORDINARY RANK FOUR GALOIS REPRESENTATIONS}

\section{REFERENCES}

AM04 A. Abbès and A. Mokrane, Sous-groupes canoniques et cycles évanescents p-adiques pour les variétés abéliennes, Publ. Math. Inst. Hautes Études Sci. 99 (2004), 117-162.

AG03 F. Andreatta and C. Gasbarri, The canonical subgroup for families of abelian varieties, Preprint (2003).

And87 A. Andrianov, Quadratic forms and Hecke operators (Springer, Berlin, 1987).

BR92 D. Blasius and J. Rogawski, Tate classes and arithmetic quotients of the two-ball, in The zeta functions of Picard modular surfaces (Université Montréal, Montréal, QC, 1992), 421-444.

BR93 D. Blasius and J. Rogawski, Motives for Hilbert modular forms, Invent. Math. 114 (1993), 55-87.

BR94 D. Blasius and J. D. Rogawski, Zeta functions of Shimura varieties, in Motives, Seattle, WA, 1991, Proceedings of Symposia in Pure Mathematics, vol. 55, part 2 (American Mathematical Society, Providence, RI, 1994), 525-571.

Bor79 A. Borel, Automorphic L-functions, Proceedings of Symposia in Pure Mathematics, vol. 33, part 2 (American Mathematical Society, Providence, RI, 1979), 27-61.

BGR84 S. Bosch, U. Grauert and R. Remmert, Non-archimedean analysis. A systematic approach, Grundlehren der Mathematischen Wissenschaften, vol. 261 (Springer, Berlin, 1984).

BCDT01 C. Breuil, B. Conrad, F. Diamond and R. Taylor, On the modularity of elliptic curves over Q: wild 3-adic exercises, J. Amer. Math. Soc. 14 (2001), 843-939.

BT99 K. Buzzard and R. Taylor, Companion forms and weight one forms, Ann. of Math. (2) 149 (1999), 905-919.

Cas W. Casselman, Introduction to the theory of admissible representations of p-adic reductive groups, http://www.math.ubc.ca/people/faculty/cass/research.html.

Cha85 C.-L. Chai, Compactification of Siegel moduli schemes, London Mathematical Society Lecture Notes Series, vol. 107 (Cambridge University Press, Cambridge, 1985).

Clo90 L. Clozel, Motifs et formes automorphes: applications du principe de fonctorialité, in Automorphic forms, Shimura varieties and L-functions, I, eds L. Clozel and J. S. Milne (Academic Press, Reading, MA, 1990), 77-159.

DS74 P. Deligne and J.-P. Serre, Formes modulaires de poids 1, Ann. Sci. École Norm. Sup. (4) 7 (1974), 507-530.

Fal90 G. Faltings, Crystalline cohomology and p-adic Galois representations, in Proceedings of the JAMI inaugural Conference on Algebraic Analysis, ed. J.-I. Igusa (Johns Hopkins University Press, Baltimore, MD, 1990).

FC90 G. Faltings and C. L. Chai, Degeneration of Abelian varieties, Ergebnisse der Mathematik und ihrer Grenzgebiete (3), vol. 22 (Springer, Berlin, 1990).

GT05 A. Genestier and J. Tilouine, Systèmes de Taylor-Wiles pour GSp(4), in Formes Automorphes (II): $G S p(4)$, Astérisque 302 (2005).

HT04 M. Harris and R. Taylor, Deformations of automorphic Galois representations, Preprint (2004).

Hid90 H. Hida, Nearly ordinary Hecke algebras and Galois representations of several variables, in Proceedings of the JAMI inaugural Conference on Algebraic Analysis, ed. J.-I. Igusa (Johns Hopkins University Press, Baltimore, MD, 1990).

Hid02 H. Hida, Control theorems of coherent sheaves on Shimura varieties of PEL type, J. Inst. Math. Jussieu 1 (2002), 1-76.

Hol95 R.-P. Holzapfel, The ball and some Hilbert problems (Birkhäuser, Basel, 1995).

Kat72 N. Katz, p-adic properties of modular schemes and modular forms, in Modular forms of one variable III, Lecture Notes in Mathematics, vol. 350 (Springer, Berlin, 1972), 69-189.

KL05 M. Kisin and K.-F. Lai, Overconvergent Hilbert modular forms, Amer. J. Math. 127 (2005), $735-783$.

LZ K. F. Lai and C. L. Zhao, Overconvergent p-adic Siegel modular forms, Preprint.

LR92 R. P. Langlands and D. Ramakrishnan, The zeta functions of Picard modular surfaces (Université de Montréal, Centre de Recherches Mathématiques, Montréal, QC, 1992). 


\section{NEARLY ORDINARY RANK FOUR GALOIS REPRESENTATIONS}

Lau96 G. Laumon, Sur la cohomologie à supports compacts des variétés de Shimura pour GSp $(4)_{/ \mathbb{Q}}$, Compositio Math. 105 (1996), 267-359.

Lau05 G. Laumon, Sur les fonctions zêtas des variétés de Siegel de dimension 3, in Formes Automorphes II: Le cas du groupe GSp(4), Astérisque 302 (2005).

MT02 A. Mokrane and J. Tilouine, Cohomology of Siegel varieties with p-adic integral coefficients and applications, Astérisque 280 (2002).

Mum70 D. Mumford, Abelian varieties (Oxford University Press, Oxford, 1970).

Per94 B. Perrin-Riou, Représentations p-adiques ordinaires, in Périodes p-adiques, Astérisque 223 (1994), 185-220.

Ram04 D. Ramakrishnan, Irreducibility of $\ell$-adic representations associated to regular cusp forms on $G L(4) / \mathbb{Q}$, Preprint (2004).

Rob01 B. Roberts, Global L-packets for GSp(2) and theta lifts, Documenta Math. 6 (2001), 247-314.

Rog90 J. Rogawski, Automorphic representations of unitary groups in three variables, Annals of Mathematics Studies, vol. 123 (Princeton University Press, Princeton, NJ, 1990).

Sch05 R. Schmidt, Iwahori-spherical representations of $G S p(2)$ and modular forms with square-free level, J. Math. Soc. Japan 57 (2005), 259-293.

Ser62 J.-P. Serre, Endomorphismes complètement continus des espaces de Banach p-adiques, Publ. Math. Inst. Hautes Études Sci. 12 (1962), 69-85.

Shi63 G. Shimura, On analytic families of polarized abelian varieties and automorphic functions, Ann. of Math. (2) 78 (1963), 149-192.

Shi78 G. Shimura, The arithmetic of automorphic forms with respect to a unitary group, Ann. of Math. (2) 107 (1978), 569-605.

Tay93 R. Taylor, On the $\ell$-adic cohomology of Siegel threefolds, Invent. Math. 114 (1993), 289-310.

TW95 R. Taylor and A. Wiles, Ring-theoretic properties of certain Hecke algebras, Ann. of Math. (2) 141 (1995), 553-572.

Til96 J. Tilouine, Deformations of Galois representations and Hecke algebras (Narosa, New Delhi, 1996).

Til06 J. Tilouine, Siegel varieties and p-adic Siegel modular forms, to appear in the special volume of Documenta Math. for the 60th birthday of J. Coates.

TU99 J. Tilouine and E. Urban, Several variable p-adic families of Siegel-Hilbert cusp eigensystems and their Galois representations, Ann. Sci. École Norm. Sup. (4) 32 (1999), 499-574.

Urb05 E. Urban, Sur les représentations p-adiques associées aux représentations cuspidales de GSp $(4)_{\mathbb{Q}}$, in Formes Automorphes (II): GSp(4), Astérisque 302 (2005).

Wei05 R. Weissauer, Four dimensional Galois representations, in Formes Automorphes (II): GSp(4), Astérisque 302 (2005).

Wil95 A. Wiles, Modular elliptic curves and Fermat's Last Theorem, Ann. of Math. (2) 142 (1995), 443-551.

Yos80 H. Yoshida, Siegel modular forms and the arithmetic of quadratic forms, Invent. Math. 60 (1980), $193-248$

J. Tilouine tilouine@math.univ-paris13.fr

Département de Mathématiques, UMR 7539, Institut Galilée, Université de Paris 13,

93430 Villetaneuse, France

Don Blasius blasius@math.ucla.edu

UCLA Mathematics Department, Box 951555, Los Angeles, CA 90095-1555, USA 